\title{
Plane Spanners of Degree Eight
}

by

\section{Darryl Hill}

\author{
A Thesis submitted to \\ the Faculty of Graduate Studies and Research \\ in partial fulfilment of \\ the requirements for the degree of \\ Master of Science \\ in \\ Computer Science \\ Carleton University \\ Ottawa, Ontario, Canada
}

May 2015

Copyright (C)

2015 - Darryl Hill 


\section{Abstract}

Given a set of points $P$, we calculate the Delaunay triangulation of $P$, labeled $D T(P)$. We describe an algorithm that selects a subset of edges from $D T(P)$ to form the graph $D 8(P)$. We then prove that $D 8(P)$ has a constant spanning ratio of $\approx 4.414$ with respect to the complete graph, and a maximum degree of 8 . 


\section{Acknowledgments}

I would like to thank my supervisors Prosenjit Bose and Michiel Smid for helping me turn some half-baked, poorly thought out ideas into a thesis. Any sections that read coherently are from their influence, and any nonsensical ramblings I claim as my own.

Thanks to Anil Maheshwari, for helping identify problems and then work through them with me, and thanks to Vida Dujmovic who graciously and selflessly agreed to be on my thesis committee.

Thank you to my wife AnnMarie for her continued support, and my children, Nolan and Garrett for being both my motivation and a welcome distraction from school work. My father Richard, my mother Paulina, and my brothers Lewis and Aaron, thank you for everything.

Many thanks go out to the computational geometry lab at Carleton University, both students and professors, for support, friendship, and for setting a standard for excellence that I one day hope to live up to. 


\section{Table of Contents}

\begin{tabular}{ll}
\hline Abstract & ii
\end{tabular}

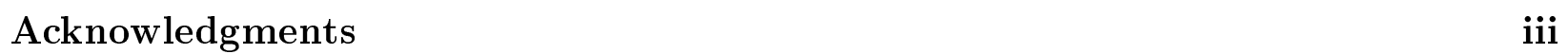

Table of Contents $\quad$ iv

List of Tables $\quad$ vi

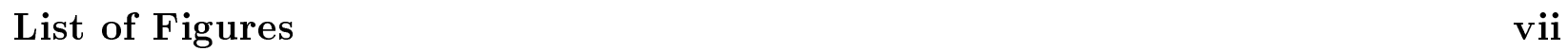

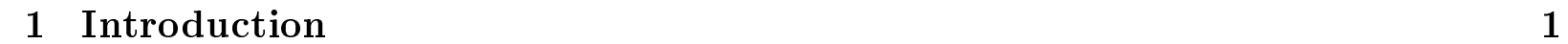

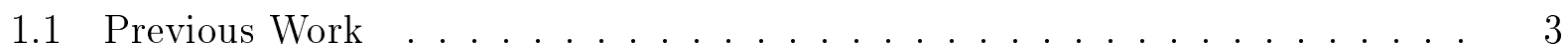

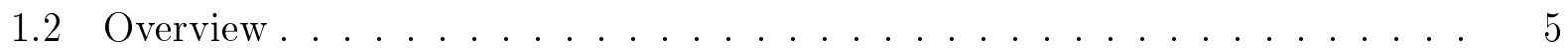

\begin{tabular}{|lll|}
\hline 2 & General Definitions & 7 \\
\hline
\end{tabular}

2.1 Graphs . . . . . . . . . . . . . . . . . . . . 7

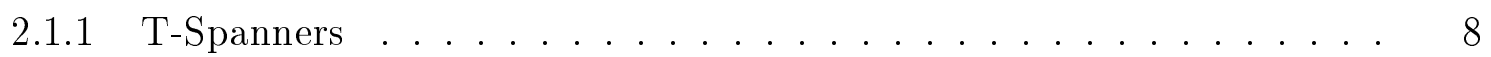

2.1 .2 Delaunay Triangulations . . . . . . . . . . . . . . . 9

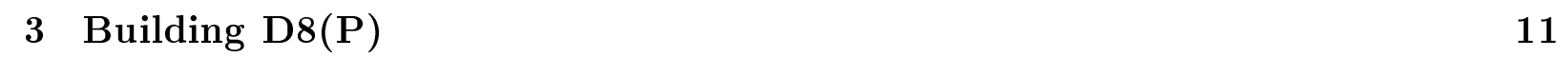

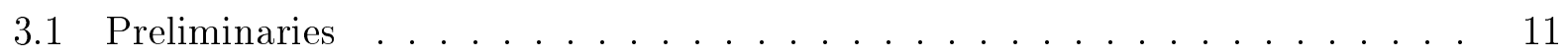

3.2 The Algorithm . . . . . . . . . . . . . . . . 15

$3.2 .1 \quad$ Constructing $\mathrm{D} 8(\mathrm{P})$. . . . . . . . . . . . . . . . 15

3.2 .2 AddIncident $(\mathrm{L})$. . . . . . . . . . . . . . . . 16 


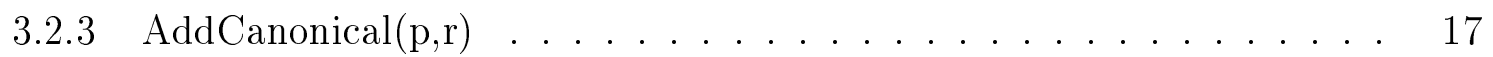

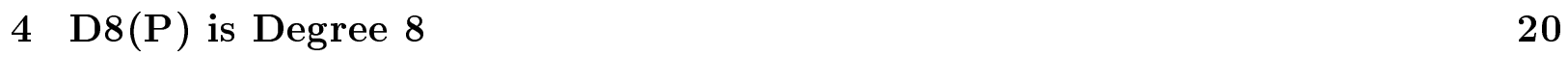

4.1 Introduction . . . . . . . . . . . . . . . . . . 20

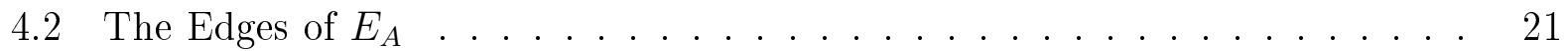

4.3 The Edges of $E_{C A N} \ldots \ldots \ldots \ldots \ldots \ldots \ldots \ldots$

4.3 .1 Cone Types . . . . . . . . . . . . . . . . . . . 21

4.3 .2 Cones in Neighbourhoods . . . . . . . . . . . . . . . 23

4.3 .3 Cones in Shared Triangles . . . . . . . . . . . . . . . . . . . 27

4.3 .4 Empty Cones . . . . . . . . . . . . . . . . 30

4.4 Charging Scheme for $\mathrm{D} 8(\mathrm{P})$

4.5 Proving the Degree of $\mathrm{D} 8(\mathrm{P}) \ldots \ldots \ldots \ldots \ldots$

4.5 .1 Preliminaries . . . . . . . . . . . . . . . 38

4.5 .2 Edges in Boundary Cones . . . . . . . . . . . . . . . . 38

$4.5 .3 \quad$ Edges in Shared Triangles . . . . . . . . . . . . . . . . . . 42

4.5 .4 The Degree of $\mathrm{D} 8(\mathrm{P})$. . . . . . . . . . . . . . . 43

$\begin{array}{lll}5 & \mathrm{D} 8(\mathrm{P}) \text { is a Spanner } & 46\end{array}$

5.1 Introduction and Preliminaries . . . . . . . . . . . . . . 46

5.2 Spanning Ratio of Ideal Paths . . . . . . . . . . . . . . . 48

$5.3 \quad$ Lengths of Paths in $D 8(P)$. . . . . . . . . . . . . . . . 63

5.3 .1 Introduction . . . . . . . . . . . . . . 63

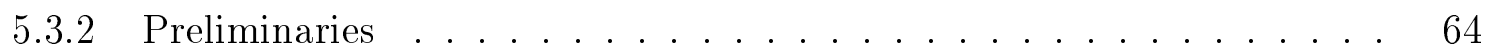

5.3 .3 Paths in $\mathrm{D} 8(\mathrm{P})$

5.4 The Spanning Ratio of $\mathrm{D} 8(\mathrm{P})$

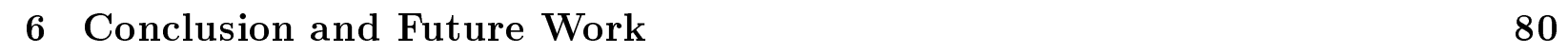

\begin{tabular}{ll}
\hline List of References & 82
\end{tabular} 


\section{List of Tables}

$1 \quad$ Known results for bounded degree planar spanners. . . . . . . . . . . . . . 4 


\section{List of Figures}

$1 \quad$ An example of how cones of a point are labeled. . . . . . . . . . . . 12

2 The bisector distance from $[p q]=\left|p q^{\prime}\right|$, where $q^{\prime}$ lies on the bisector of the cone. 13

$3 \quad$ The cone neighbourhood $N_{i}^{p} . \ldots \ldots \ldots \ldots \ldots \ldots$

$4 \quad$ Canonical edges. . . . . . . . . . . . . . . . . . . . . . 14

$5 \quad$ AddIncident $(L)$ selects edge $(p, r)$ for $E_{A} . \ldots \ldots \ldots \ldots$

$6 \quad$ The graph $C a n_{0}^{p}$, based on $(p, r) \in E_{A}$, in red. . . . . . . . . . 17

$7 \quad$ AddCanonical $(p, r) \ldots \ldots \ldots \ldots \ldots \ldots$

$8 \quad$ Regions and cones. . . . . . . . . . . . . . . . . . . . 22

$9 \quad$ Properties of convex quadrilaterals in $D T(P) . \ldots \ldots \ldots \ldots \ldots$

$10 \quad$ Consecutive canonical edges have an angle facing $p$ of at least $2 \pi / 3$, and thus if $(p, r) \notin E_{A}$, there is at least one empty cone between them in $D 8(P)$. . . 26

11 Lemma|4.3.10 . . . . . . . . . . . . . . . . . . . . . . 27

$12 \quad$ A triangle shared by $N_{0}^{p}$ and $N_{3}^{p^{\prime}} . \ldots \ldots \ldots \ldots \ldots \ldots \ldots \ldots \ldots \ldots \ldots \ldots$

$13 q \in C_{0}^{p} \cap C_{3}^{p^{\prime}}$ violates the empty circle property of Delaunay triangulations. . 29

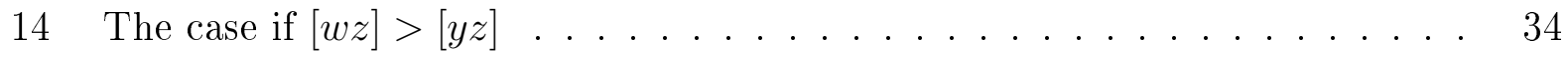

15 Vertex $s$ is an inner vertex of $\operatorname{Can}_{0}^{(p, r)}, s \neq r$. Charge $C_{2}^{s} \ldots \ldots . . . .34$

$16 \quad$ Vertex $s$ is neither the first nor last vertex of $\operatorname{Can}_{0}^{(p, r)}, s \neq r . \ldots . . .35$

17 Vertex $s$ is neither the first nor last vertex of $\operatorname{Can}_{0}^{(p, r)}, s \neq r . \ldots . . .35$

$18 \quad(p, r) \in E_{A}$, canonical edges with endpoint $r . \ldots \ldots \ldots \ldots$

$19 \quad(y, z)$ is the last canonical edge in $\operatorname{Can}_{0}^{(p, r)}$, and is charged to $C_{4}^{z} . \ldots 36$

$20 \quad(y, z)$ is the last canonical edge in $\operatorname{Can}_{0}^{(p, r)}$, and is charged to $C_{4}^{z} \ldots \ldots 37$ 
$21 \quad(w, y)$ is charged to $y$ in place of $(y, z) . \ldots \ldots \ldots \ldots \ldots \ldots$

$22 \quad(w, y)$ is charged to the empty cone of $w \ldots \ldots \ldots \ldots \ldots$

23 There must be an edge of $E_{A}$ between $(y, z)$ and $\left(y^{\prime}, z\right) \ldots \ldots \ldots$

24 If $(y, z)$ is not in $E_{A}$, there must be a neighbour of $y$ or $z$ in $\triangle(y x z) . \ldots 41$

25 Only $(p, s)$ can be added to $E_{C A N}$ in $\mid$ Step $4 c \mid$ with apex $q . \quad \ldots \ldots . . .43$

26 Only $\left(p^{\prime}, s\right)$ can be added to $E_{C A N}$ in $\mid$ Step $4 c \mid$ with apex $q . \ldots . . . .44$

$27 \quad$ A degree 8 vertex in $D 8(P)$. The red edges belong to $E_{C A N}$, while the black edges belong to $E_{A}$. The light edges are edges of $D T(P)$ that may or may not

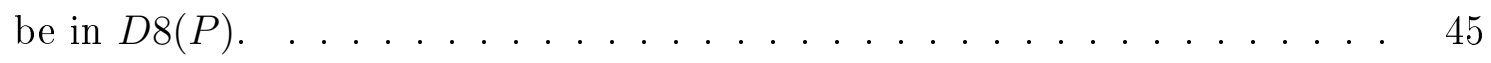

$28 \quad$ The canonical triangle $T_{p q}$ in blue. Note, a possible canonical path (in red) does not need to be inside the canonical triangle. . . . . . . . . . 47

29 The Inscribed Angle Theorem $\ldots \ldots \ldots$. . . . . . . . . . . . 49

$30 \quad$ Length of arc between $r$ and $q . \ldots \ldots \ldots \ldots \ldots$

31 If there is a point $t$, then $(p, r)$ is not an edge in $D T(P) . \ldots \ldots \ldots \quad 51$

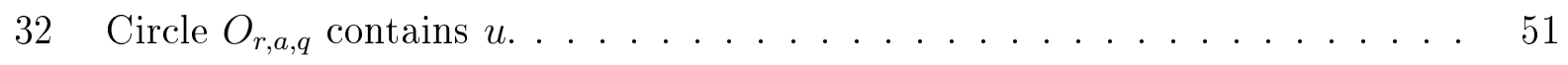

33 A circle through $a$ and $p$ contains $u$ or $v$. . . . . . . . 53

34 Lemmat5.2.61 . . . . . . . . . . . . . . . . . 56

$35 \quad$ If $|p a| \geq|p q|$, apply Lemma $|5.2 .6| \ldots \ldots \ldots \ldots \ldots \ldots \ldots$

36 If the vertex $a$ is in $\triangle(p r q)$, we proceed by induction from $r$ to $a$. . . . . 58

37 Proceed by induction from $a$ to $q . \ldots \ldots \ldots \ldots \ldots \ldots$

$38 \quad\left|r_{a} a\right| \frac{\alpha_{1}}{\sin \alpha_{1}}<\left|r_{a} a\right| \frac{\alpha}{\sin \alpha}$ and $\left|a_{q} q\right| \frac{\alpha_{2}}{\sin \alpha_{2}}<\left|a_{q} q\right| \frac{\alpha}{\sin \alpha} \ldots \ldots \ldots \ldots \ldots . \ldots \ldots 60 . \ldots \ldots$

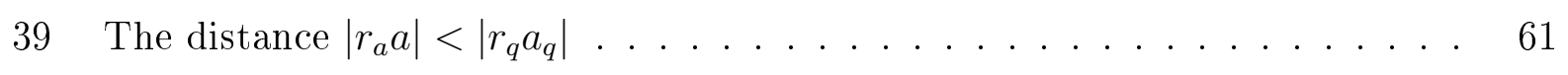

$40 \quad$ The distances across the arcs are $\left|r_{q} a_{q}\right| \frac{\alpha}{\sin \alpha}+\left|a_{q} q\right| \frac{\alpha}{\sin \alpha}=\left|r_{q} q\right| \frac{\alpha}{\sin \alpha} \ldots \ldots \quad 61$

41 Lemma $5.2 .81 \ldots \ldots \ldots$. . . . . . . . . . . . . . . . . . . . 62

$42 \quad$ An example arrangement of vertices. $\ldots \ldots \ldots \ldots$. . . . . . . . . 64

$43 \quad$ Any edge with endpoint $p$ in $N_{i}^{p}$ clockwise from $(p, q)$ must be longer than $[p q]$. 65

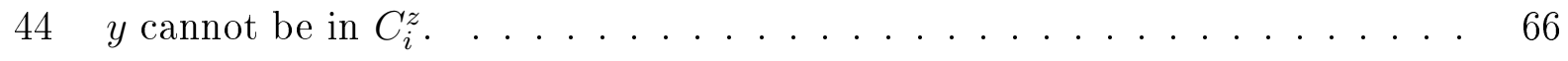

45 If the final edge of $\mathrm{Can}_{0}^{p}$ is in $C_{0}^{y}$, we do not add it to $E_{C A N} . \ldots \ldots 67$ 
$46 \quad$ Ideal paths from $p$ to $y$ and $z$ to $y . \ldots \ldots$. . . . . . . . . 68

47 The path corresponding to Equation $|5.3 .1|$ Lemma $\mid 5.3 .4$. . . . . . . . . . . 69

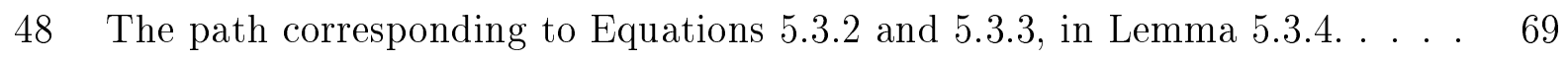

49 The green path is the length of the path we can prove, the red path is how much path we have to pay for it. . . . . . . . . . . . 71

$50 \quad$ Notice that the inductive path takes the longer path between $a_{2}$ and $b_{2}$. . $\quad 71$

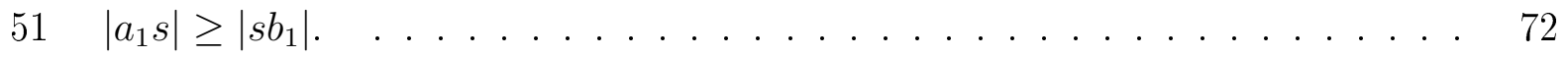

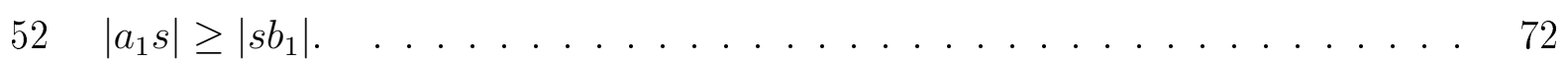

$53 \quad$ AddCanonical $(p, r) \ldots \ldots \ldots \ldots \ldots \ldots$ 


\section{Chapter 1}

\section{Introduction}

We want to go for a drive in our car. We are at home, which is our starting point, and we have a specific destination in mind.

Between these two locations are a system of roads. These roads intersect each other at discrete locations. Each road segment takes us from one location to another, whether that location is the start of our journey, the end of our journey, or an intersection along the way.

The structure of this network of roads shapes the way we interact with them in a specific way. When giving directions, we do not bother the details of how to drive. We don't say "stay between the lines, obey traffic laws, don't run in to other cars." Rather, we give a set of simple instructions. We say, for instance, take road 1 until it intersects road 2. Turn right on road 2 and drive until it intersects road 3. Take a left on road 3 until you come to the destination. We may give details such as how long to expect to drive down any given road, which is a rough estimate of the length of the road. Additionally, often without thinking, if we are giving directions or planning a route, we give the shortest path possible.

Thinking of roads in this way allows us to communicate directions simply, and understand directions when given to us. It allows us to package a lot of useful information in a few short details. In this way, without being conscious of it, we have modeled the system of roads as a graph.

Informally a graph consists of a set of points, and links between them that we refer to as edges. The above example deals with points that are physical locations, and has edges that 
have a distance associated with them. This makes what we refer to as a geometric graph, which we define more formally in Chapter 2 .

In the case of a road network, we are mainly concerned with traversing the graph, and we often wish to traverse it in a manner that will minimize the distance traveled. If the path from source to destination consists of a single, straight road, then the distance traveled is what we call the Euclidean distance, which is the shortest distance between two points. If there was a straight road between every possible pair of locations (a highly impractical solution), then the road network would consist of the complete graph, and we could always guarantee that the distance we travel between two locations is the shortest possible.

Of course such a road network is prohibitively expensive and impractical. Though we don't always have such a path, we try to approximate it as much as possible. A road network is good if the path between source and destination only deviates a reasonable amount from the shortest distance possible. If we can make guarantees that we never deviate from the shortest distance more than some number, say $t$, times the Euclidean distance, our road network would be a spanning graph of the complete graph, or a $t$-spanner.

There are other methods to evaluate a road network. Cost factors in highly; as such we would like an efficient road network, where we in a sense minimize the number of roads while still maintaining favourable spanning properties. Additionally, at each intersection or location, we would like to minimize the number of roads that start or end there. It limits congestion, and avoids the need for a large number of expensive traffic lights. In general we do not wish to have more a small number of road segments meeting at an intersection. In graph terminology, we would refer to the number of roads that start or end at a location as the degree of that location. Clearly to be a good road network, we want favourable spanning properties and a bounded degree.

Clearly there are other graph applications with similar needs. Computer networks need to allow packets to travel from one location to another in an efficient manner. Having too many paths going through the same location (high degree) means increased congestion and/or expensive infrastructure to handle the higher volume. Limiting the degree of a node 
while maintaining favourable routing conditions gives us an efficient network that minimizes costs.

In this thesis we focus on the construction and analysis of bounded degree spanning graphs.

We take the paper of Bonichon et al. [1], who takes a graph known as the half- $\theta-6$ graph, and creates a subgraph that is a bounded degree planar spanner. We take the techniques used and apply them on another graph, the Delaunay triangulation. In applying to the Delaunay triangulation, we borrow amply from results of Bose et. al [2], as well as Kanj et al. [3], both of whom construct bounded degree planar subgraphs of the Delaunay triangulation which are still $t$-spanners. Using a combination of these methods, we construct a subgraph of the Delaunay triangulation that has a maximum degree of 8 and has the best spanning ratio of any known bounded degree planar graph with degree less than 14 .

\subsection{Previous Work}

The original spanning ratio result was on Delaunay triangulations in the L1 metric by Chew [4], who proved a spanning ratio of $\sqrt{10}$. Dobkin at al. 5 found a result of the Delaunay triangulation of the L2 metric, getting a ratio of $\frac{1+\sqrt{5}}{2} \pi$ using the Delaunay triangulations relation to the Voronoi diagram. This was then improved by Keil $|6|$ to $\frac{2 \pi}{3 \cos \left(\frac{\pi}{6}\right)}$, and finally taken to its current known spanning ratio of 1.998 by Xia 7$]$.

Chew [4] conjectured the lower bound on the spanning ratio of the Delaunay triangulation was $\frac{\pi}{2}$, until Bose et. al $|8|$ proved that almost all Delaunay triangulations had a spanning ratio of greater than $\frac{\pi}{2}$, by proving a lower bound of 1.5846. Xia [9] recently found a point set with a spanning ratio of 1.5932 , and conjectured that the tight bound would be found at the limit of a point set in a similar configuration, when the number of points approached infinity.

There are also a number of results on bounded degree planar spanners. We give a brief overview of some of the results in Table 1 . 


\begin{tabular}{|c|c|c|}
\hline Paper & Degree & Stretch Factor \\
\hline \hline Bose et al. |10| & 27 & $(\pi+1) C_{O} \approx 8.27$ \\
Li \& Wang |11| & 23 & $\left(1+\pi \sin \left(\frac{\pi}{4}\right)\right) C_{O} \approx 6.44$ \\
Bose et al. |12| & 17 & $\left(\frac{1+\sqrt{3}+3 \pi}{2}+2 \pi \sin (\pi / 12)\right) C_{O} \approx 23.58$ \\
Kanj et al. $|3|$ & 14 & $\left(1+\frac{2 \pi}{14 \cos (\pi / 14)}\right) C_{O} \approx 2.92$ \\
Bose et al. $|2|$ & 7 & $\left(\frac{1}{1-2 \tan (\pi / 8)}\right) C_{O} \approx 11.65$ \\
Bose et al. $|2|$ & 6 & $\left(\frac{1}{(1-\tan (\pi / 7)(1+1 / \cos (\pi / 14))} C_{O} \approx 81.66\right.$ \\
Bonichon et al. $|1|$ & 9 & 6 \\
Bonichon et al. $|1|$ & 6 & $\left(1+\frac{2 \pi}{6 \cos (\pi / 6)}\right) C_{O} \approx 4.41$ \\
Bonichon et al. $\mid 13$ & 4 & $\sqrt{4+2 \sqrt{2}(19+29 \sqrt{2}) \approx 156.82}$ \\
\hline \hline This paper & 8 & $\left(1+\frac{2 \pi}{14 \cos (\pi / 14)}\right) C_{O} \approx 2.92$ \\
\hline Kanj et al. $|3|$ & 14 & $C_{O}=1.998|7|$
\end{tabular}

Table 1: Known results for bounded degree planar spanners.

Bounded degree planar spanners are often obtained by taking a subset of edges of an existing planar spanner that has bounded degree, while maintaining spanning properties. Using this technique, the properties of the underlying graph become very important. For instance, if we have a subgraph that is a $t^{\prime}$-spanner, and the original graph was a $t$-spanner, then certainly $t^{\prime} \geq t$. Thus, if we want a subgraph with a good spanning ratio, we want to take a subgraph of a graph with a good spanning ratio. We note how in Table 1 that most of the results are subgraphs of the Delaunay triangulation, which has the best known spanning ratio of any planar graph, currently $1.998|7|$. We note that Bonichon et al. |1| have good results using the half- $\theta 6$ graph, which has a spanning ratio of 2.

The research into bounded degree spanners begins with Bose et al, [10 and a subgraph of the Delaunay triangulation that has a maximum degree of 27. Li \& Wang [11 achieve a degree 23 graph with an improved spanning ratio, in which we see the degree bound reduced from 27 to 23 while maintaining respectable spanning properties. Bose et al, [12 responded with a degree 17 graph, at the cost of an increase in the spanning ratio. 
Then, notably, Kanj et al. [3] improve the degree to 14 while having the lowest spanning ratio yet, $\approx 2.92$. While their claim to the lowest spanning ratio of bounded degree planar graph still stands, their degree bound of 14 has been subsequently improved upon. Bose et al. [2] improved the degree bound on Delaunay triangulation subgraphs to 7 and 6 , but at the cost of an inflated spanning ratio of $\approx 11.65$ and $\approx 54.22$ respectively. Bonichon et al. [1] managed similar degree bounds, most notably matching the degree bound of 6 , while also managing to improve the spanning ratio to 6 . It is notable that in their paper, Bonichon et al. eschew the seemingly traditional practice of sub-graphing the Delaunay triangulation, and instead opting to use the half- $\theta-6$ graph. The natural partitioning of edges of the half- $\theta-6$ graph make it something of a natural for selecting a bounded degree subgraph.

Bonichon et al. [13] additionally obtain the lowest degree planar spanner to date, with a degree bound of 4 . However they pay a moderate price in their spanning ratio of $\approx 156.82$.

Our results take a philosophy similar to Bonichon et al. [1], in that there is value in attempting to optimize both degree and spanning ratio. As such obtain a respectable degree 8 , with a highly competitive spanning ratio. It is the lowest spanning ratio of any graph of degree less than 14. Kanj et al. [3] have the best known spanning ratio of bounded degree planar graphs for degree 14 and under. They obtain a degree of 14 by dividing the area around a vertex into 14 equal angled "cones". We take a similar approach, dividing the area around a vertex into 6 cones. Our spanning ratio matches that of Kanj and Perkovic [3] when corrected for cone angle, although our degree is 2 higher than our number of cones. The similarities are not simply superficial; our initial analysis of path length in a bounded degree Delaunay subgraph is almost completely modeled from their analysis.

\subsection{Overview}

In Chapter 2, we provide general definitions of terms that will be used throughout this thesis. In Chapter 3 we describe how to select a subset of the edges of the graph $D T(P)$ to form the graph $D 8(P)$. In Chapter 4 we prove that $D 8(P)$ has a maximum degree of 8 . In Chapter 
5 we bound the spanning ratio of $D 8(P)$ with respect to $D T(P)$. Since $D T(P)$ is a spanner of the complete Euclidean graph, this makes $D 8(P)$ a spanner of the complete Euclidean graph as well. Finally in Chapter 6 we draw conclusions based on our work, and examine some potential directions that related research might extend. 


\section{Chapter 2}

\section{General Definitions}

Now that we have looked into the background of the problem, we will give precise definitions of the terms we will be using.

\section{$2.1 \quad$ Graphs}

Definition 2.1.1. A graph $G=(V, E)$ consists of a finite set of vertices $V$, and a set E of edges, where each edge is an unordered pair of vertices. We also use the alternative notation of $G(V)$, which means a graph $G$ induced on the vertices $V$.

Definition 2.1.2. A graph is called planar if it can be drawn in the 2-dimensional plane as a set of points (vertices) and line segments (edges) such that no two edges cross except at a common endpoint. Such a drawing is called an embedding.

Definition 2.1.3. A plane graph is a graph that has been embedded in the plane such that no two edges cross.

Plane graphs may have curved edges, but for the purposes of this thesis we will assume all edges are straight line segments.

Definition 2.1.4. A geometric graph is a graph in which the edges and vertices are 
associated with geometric objects or measurements.

Definition 2.1.5. A Euclidean graph is a geometric graph in which the vertices represent points in the plane, and the edges are assigned lengths equal to the Euclidean distance between those points.

All graphs discussed in this thesis are Euclidean graphs.

Definition 2.1.6. Consider a graph $G=(V, E)$. The degree of a vertex $v \in V$ is the number of edges $e \in E$ incident to $v$. Alternatively it is the number of edges $e \in E$ that $v$ is the endpoint of.

As discussed earlier, we are interested in graphs where the degree of any vertex is not too high. Formally:

Definition 2.1.7. A bounded degree graph is a graph $G=(V, E)$ where, for any $v \in V$, the degree of $v$ is no greater than some value $d$.

\subsubsection{T-Spanners}

A $t$-spanner is a Euclidean graph that has the property that the shortest path between any two vertices is no more than a constant $t$ times longer than the Euclidean distance between those vertices. Formally we say:

Definition 2.1.8. Let $t \geq 1$ be any real number. Then a Euclidean graph $G$ is a $t$ spanner if, for any two vertices $p$ and $q$ in the graph, the shortest distance from $p$ to $q$ through the edges of $G$, denoted $\delta_{G}(p, q)$, is no more than $t$ times the Euclidean distance from $p$ to $q$. That is: 


$$
\frac{\delta_{G}(p, q)}{|p q|} \leq t
$$

\subsubsection{Delaunay Triangulations}

One graph that is of particular interest to us is the Delaunay triangulation. The Delaunay triangulation forms the basis of the bounded-degree spanning graphs we construct here.

The following definitions assume general position of a point set $P$. Thus no three points are on a line, and no four points are on a circle.

Definition 2.1.9. A triangulation of a point set $P$ is a plane graph of the vertex set $P$ such that we cannot add another edge between vertices of $P$ without inducing an edge crossing. If we assume all edges are straight line segments, then every face of the graph is a triangle, with the possible exception of the outer face.

Of special consideration to us is the Delaunay triangulation. The Delaunay triangulation of a set of points $P$ is denoted $D T(P)$. It is a triangulated Euclidean graph that has many favourable properties. There are several equivalent constructions of the Delaunay triangulation, but there are two in particular that are the most conducive to our analysis.

- For every triple of points of the set $P$, if the unique circle through these three points is empty of points of $P$, then we connect the three points such that the edges form a triangle.

- For every pair of points $x, y$ of the set $P$, if there exists a circle through $x$ and $y$ that is empty of any points of $P$, then we connect $x$ and $y$ with an edge.

This construction enables us to define a property of Delaunay triangulations that is of particular importance to this thesis. 
Definition 2.1.10. An edge e of a graph $G(P)$ has the empty circle property if there exists a circle through the endpoints of e that does not contain any other point of $P$.

As we can see, every edge of a graph $D T(P)$ has the empty circle property.

It is worth reviewing that, for all of its favourable properties, the degree of the Delaunay triangulation is unbounded. 


\section{Chapter 3}

\section{Building D8(P)}

Given as input a set $P$ of $n$ points in the plane, we present an algorithm for building a bounded degree plane graph with maximum degree 8 and constant spanning ratio denoted $D 8(P)$.

The graph $D 8(P)$ is a subgraph of the Delaunay triangulation of $P$, denoted $D T(P)$. While $D T(P)$ has the best known spanning ratio of any planar graph, with a spanning ratio of 1.998 [7], it does not have a bound on the degree of a vertex. Thus the focus of our algorithm is to compute a bounded degree spanning subgraph of $D T(P)$ that is still a spanner.

We start with some preliminaries, then present the algorithm.

\subsection{Preliminaries}

We assume general position of $P$; i.e, no three points are on a line, no four points are on a circle, and no two points form a line with slope $0, \sqrt{3}$ or $-\sqrt{3}$.

In our algorithm, we use the concept of cones. Cones are a partitioning of the space around every point $p \in P$ with rays, where the rays form the boundary of consecutive cones of $p$. See Figure 1 .

We describe the precise partitioning by cones used by our algorithm. The first ray from $p$ to infinity is parallel to the $x$-axis in the positive (right) direction. We then define 5 


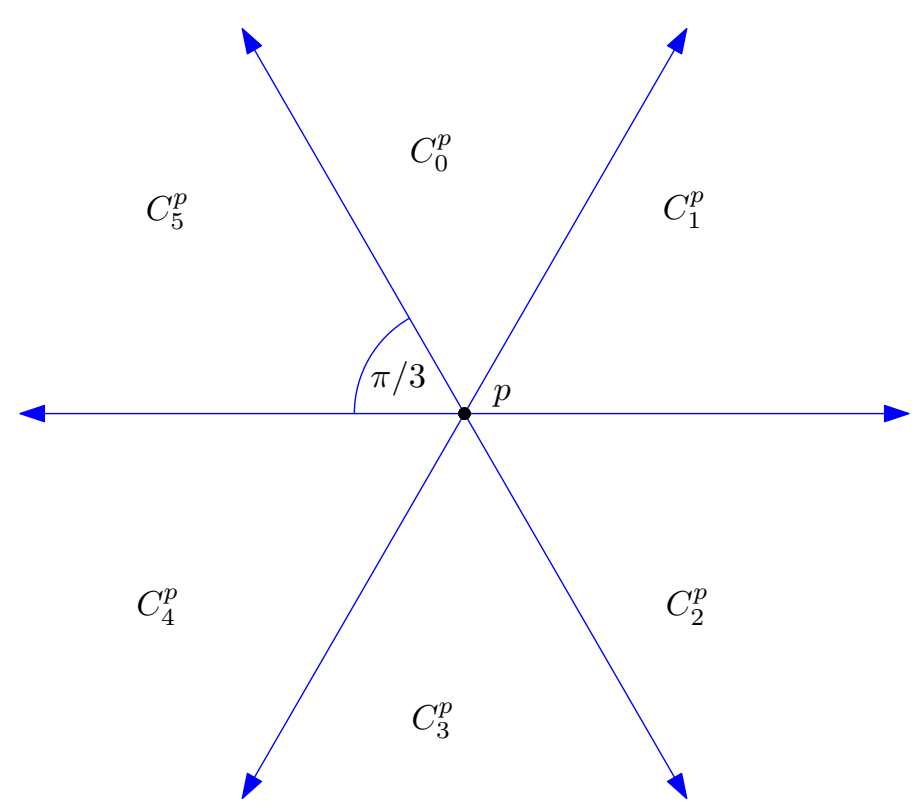

Figure 1: An example of how cones of a point are labeled.

additional rays, each starting at $p$ and going to infinity, at angular intervals of $\pi / 3$. Thus for every point $p \in P$, we partition the surrounding space into 6 cones at angular intervals of $\pi / 3$ radians each.

We number the cones starting with the topmost cone (the cone that is defined by rays at $\pi / 3$ and $2 \pi / 3$ to the $x$-axis) as $C_{0}$. If we are referring to a specific cone on a specific point $p$ we label it $C_{i}^{p}$, where $i$ is the cone index. We then number the cones by increments of one in the clockwise direction. Thus $C_{0}$ is bounded on one side by $C_{1}$ and bounded on the other side by $C_{5}$. See Figure 1 .

When referring to a cone in relation to another cone, we use an offset that is modulo 6 . Thus for cone $i$, where $i=5$, when we refer to cone $C_{i+2}^{p}$, we are referring to cone $C_{1}^{p}$.

By our general position assumption we note that no point of $P$ lies on the boundary of a cone.

In addition to Euclidean distance, there is another distance metric we use, which we call the bisector distance.

Definition 3.1.1. Given an arbitrary edge $(p, q)$, that lies in $C_{0}^{p}$, which implies that it 


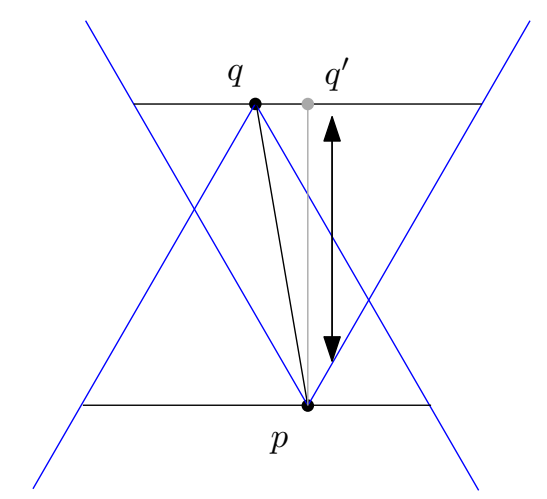

Figure 2: The bisector distance from $[p q]=\left|p q^{\prime}\right|$, where $q^{\prime}$ lies on the bisector of the cone.

is also in $C_{3}^{q}$, the bisector length of $(p, q)$ is the distance from $p$ to the orthogonal projection of $q$ onto the bisector of $C_{0}^{p}$. Denote this length as $[p q]$.

Note that the bisector of $C_{3}^{q}$ is parallel to the bisector of $C_{0}^{p}$, and thus $[p q]$ is the same whether measured from $p$ or $q$, and thus each edge of $D T(P)$ has a unique bisector length (dependent only on the orientation of the cones). See Figure 2 for an illustration.

Henceforth, any reference made to distance is to the bisector distance. When referring to Euclidean distance we state it explicitly, and use the notation $|p q|$.

Definition 3.1.2. Let $\left\{q_{0}, q_{1}, \ldots, q_{d-1}\right\}$ be the sequence of all neighbours of $p$ in $D T(P)$ in consecutive clockwise order. The neighbourhood $N_{p}$, with apex $p$, is the graph with the vertex set $\left\{p, q_{0}, q_{1}, \ldots, q_{d-1}\right\}$ and the edge set $\left\{\left(p, q_{j}\right)\right\} \cup\left\{\left(q_{j}, q_{j+1}\right)\right\}, 0 \leq j \leq d-1$, with all values mod d. The edges $\left\{\left(q_{j}, q_{j+1}\right)\right\}$ are called canonical edges. $N_{i}^{p}$ is the subgraph of $N_{p}$ induced by all the vertices of $N_{p}$ in $C_{i}^{p}$, including $p$. This is called the cone neighbourhood of $p$.

See Figures 3 , 4 . 


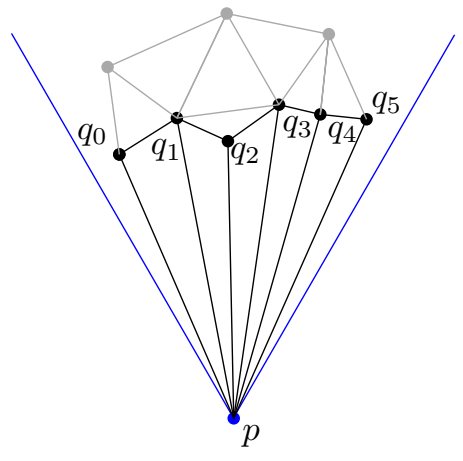

$\longrightarrow$ Cone boundaries

Figure 3: The cone neighbourhood $N_{i}^{p}$.
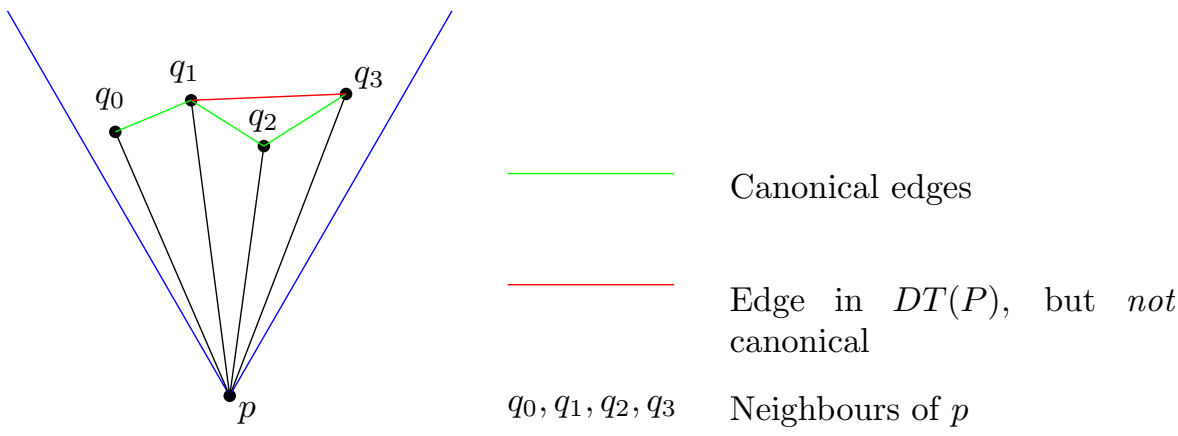

Figure 4: Canonical edges. 


\subsection{The Algorithm}

The algorithm Construct $D 8(P)$ takes as input a point set $P$ and returns the bounded degree graph $D 8(P)$, with vertex set $P$ and edge set $E$.

The algorithm calls two subroutines, AddIncident() and AddCanonical(). AddIncident () selects a set of edges $E_{A}$. For each edge $(p, r)$ of $E_{A}$, we call $\operatorname{Add} \operatorname{Canonical}(p, r)$ which adds edges to the set $E_{C A N}$. Note that both $E_{A}$ and $E_{C A N}$ are a subset of the edges in $D T(P)$.

The final graph $D 8(P)$ consists of the vertex set $P$ and the union of edge sets $E_{A}$ and $E_{C A N}$.

We now give the details of the algorithm.

\subsubsection{Constructing D8(P)}

We have as input a set of points in the plane $P$, and it provides as output a graph $D 8(P)$.

\section{Algorithm: ConstructD8(P)}

INPUT: $\quad$ Set $P$ of $n$ points in the plane in general position.

OUTPUT: $\quad D 8(P)$ : spanning subgraph of $D T(P)$ with maximum vertex degree 8 and a constant

Step 1: We compute the Delaunay triangulation $D T(P)$ of the point set $P$.

Step 2: Sort all the edges of $D T(P)$ by their bisector length, into a set $L$, in non-decreasing order.

Step 3: Initialize the set $E_{A}=\emptyset$.

Step 4: Call the function AddIncident $(L)$ with $L$ as the argument. AddIncident() selects and returns the subset $E_{A}$ of the edges of $L$.

Step 5: Iterate over the edges in $E_{A}$ in sorted order. For each edge $(p, r)$ in $E_{A}$ call AddCanonical $(p, r)$ and AddCanonical $(r, p)$. These two calls add edges to the set 


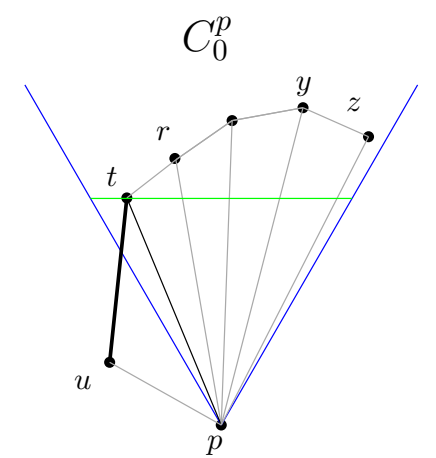

(a) Since $(t, u)$ shown in bold is in $E_{A}$, and both $u$ and $p$ are in $C_{3}^{t},(p, t)$ is not added to $E_{A}$.

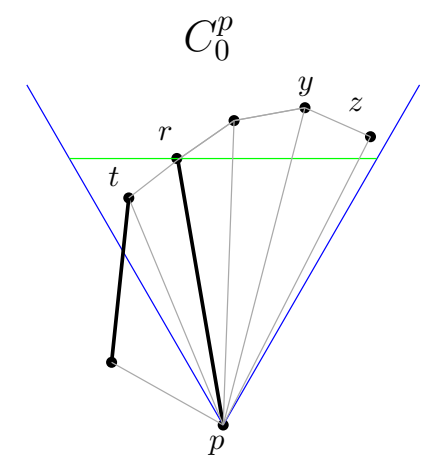

(b) AddIncident() proceeds to examine $(p, r)$, which is added to $E_{A}$.

Figure 5: AddIncident $(L)$ selects edge $(p, r)$ for $E_{A}$.

$E_{C A N}$.

Step 6: Return $D 8(P)=\left(P, E_{A} \cup E_{C A N}\right)$.

\subsubsection{AddIncident(L)}

\section{Algorithm: AddIncident(L)}

INPUT: $\quad L$ : set of edges of $D T(P)$ sorted by bisector distance.

OUTPUT: $\quad E_{A}$ : a subset of edges of $D T(P)$.

Step 1: Initialize the set $E_{A}=\emptyset$.

Step 2: For each $(p, q) \in L$, in non-decreasing order, do:

(a) Let $i$ be the cone of $p$ containing $q$. If $E_{A}$ has no edges with endpoint $p$ in $N_{i}^{p}$, and if $E_{A}$ has no edges with endpoint $q$ in $N_{i+3}^{q}$, then we add $(p, q)$ to $E_{A}$.

Step 3: return $E_{A}$.

Note that this algorithm adds, for any vertex $p \in P$, at most one edge per cone of $p$. Thus the graph $G=\left(P, E_{A}\right)$ has maximum degree 6 . 


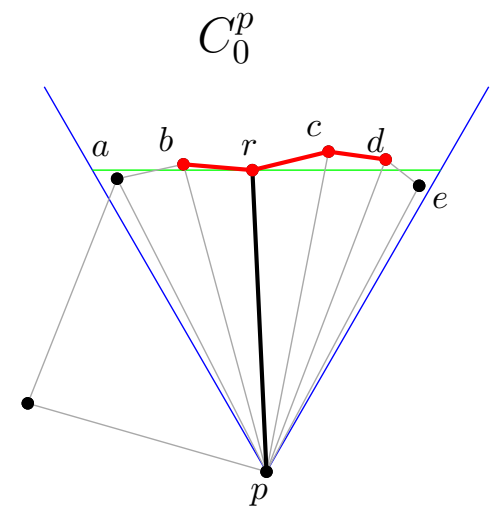

Figure 6: The graph $\operatorname{Can}_{0}^{p}$, based on $(p, r) \in E_{A}$, in red.

However, it is not clear that $G=\left(P, E_{A}\right)$ is a spanner. AddCanonical( $)$ will add edges to the graph giving us a constant spanning ratio while slightly increasing the degree to 8 .

\subsubsection{AddCanonical(p,r)}

We require the following definition:

Definition 3.2.1. Let $\operatorname{Can}_{0}^{(p, r)}$ be the subgraph consisting of the ordered subsequence of canonical edges $(s, t)$ of $N_{0}^{p}$ in clockwise order around apex $p$ such that $[p s] \geq[p r]$ and $[p t] \geq$ $[p r]$. We call $\operatorname{Can}_{0}^{(p, r)}$ a canonical subgraph. A vertex that is the first or last vertex of Can $_{0}^{(p, r)}$ is called an end vertex of $\operatorname{Can}_{0}^{(p, r)}$. A vertex that is not the first or last vertex in $\operatorname{Can}_{0}^{(p, r)}$ is called an inner vertex of $\operatorname{Can}_{0}^{(p, r)}$. Vertex $r$ is called the anchor of $\operatorname{Can}_{0}^{(p, r)}$. See Figure 6.
Algorithm: AddCanonical(p,r)
INPUT: $\quad(p, r)$, an edge of $E_{A}$.
OUTPUT: A set of edges that are a subset of the edges of $D T(P)$. All edges gener- ated by calls to AddCanonical() form the set $E_{C A N}$.

Step 1: Without loss of generality, let $(p, r)$ be an edge of $E_{A}$, where $r \in C_{0}^{p}$. 
Step 2: If there are at least three edges in $\operatorname{Can}_{0}^{(p, r)}$, then for every canonical edge $(s, t)$ in $\operatorname{Can}_{0}^{(p, r)}$ that is not the first or last edge in the ordered subsequence of canonical edges $\operatorname{Can}_{0}^{(p, r)}$, we add $(s, t)$ to $E_{C A N}$.

Step 3: If the anchor $r$ is the first or last vertex in $\operatorname{Can}_{0}^{(p, r)}$, and there is more than one edge in $\operatorname{Can}_{0}^{(p, r)}$, then add the edge of $\operatorname{Can}_{0}^{(p, r)}$ with endpoint $r$ to $E_{C A N}$. See Figure $7 \mathrm{~b}$.

Step 4: Consider the first and last canonical edge in $\operatorname{Can}_{0}^{(p, r)}$. Since the conditions for the first and last canonical edge are symmetric, we only describe how to process the last canonical edge $(y, z)$. There are three possibilities.

(a) If $(y, z) \in N_{5}^{z}$ we add $(y, z)$ to $E_{C A N}$. See Figure $7 \mathrm{c}$.

(b) If $(y, z) \in N_{4}^{z}$ and $N_{4}^{z}$ does not have an edge with endpoint $z$ in $E_{A}$, then we add $(y, z)$ to $E_{C A N}$. See Figure $7 \mathrm{~d}$

(c) If $(y, z) \in N_{4}^{z}$ and there is an edge with endpoint $z$ in $E_{A} \cap N_{4}^{z} \backslash(y, z)$, then there is exactly one canonical edge of $z$ with endpoint $y$ in $N_{4}^{z}$. We label this edge $(w, y)$ and add it to $E_{C A N}$. See Figure $7 \mathrm{e}$.

That concludes the steps in the construction of $D 8(P)$. In Chapter 4 we will prove that the maximum degree of this graph is at most 8, and in Chapter 5 we will prove that its spanning ratio is bounded by a constant. 


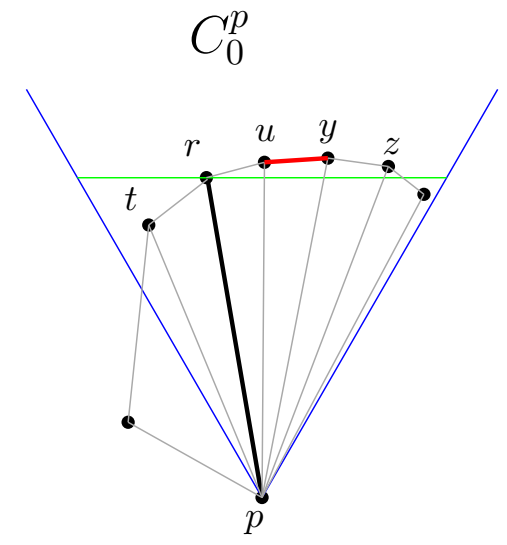

(a) Edges added to $E_{C A N}$ in Step 2.

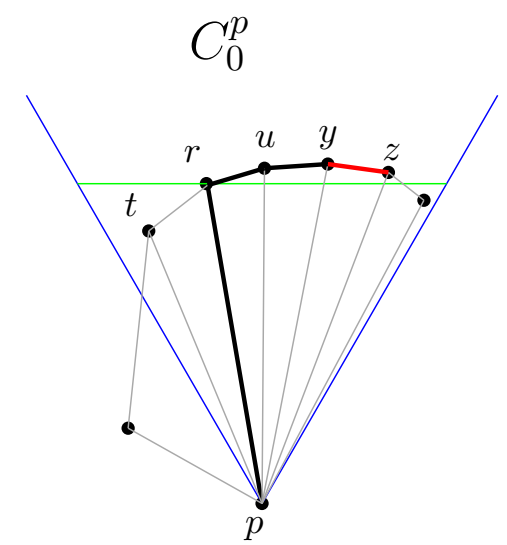

(c) Edge added to $E_{C A N}$ in Step 4 a.

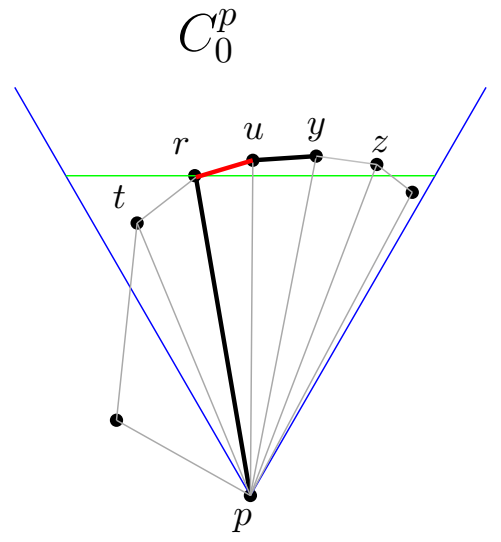

(b) Edge added to $E_{C A N}$ in Step 3.

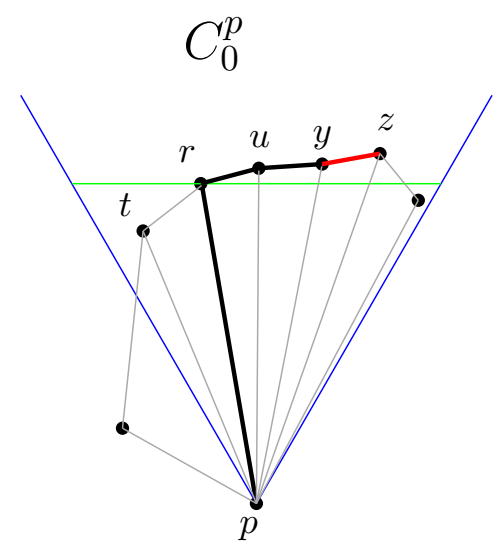

(d) Edge added to $E_{C A N}$ in Step $4 \mathrm{~b}$

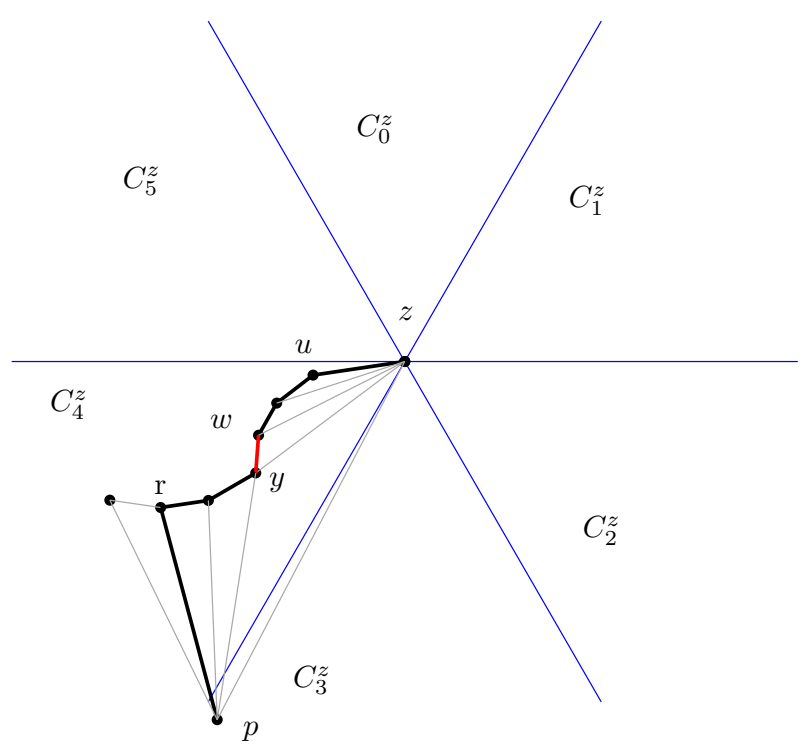

(e) Edge added to $E_{C A N}$ in Step 4c.

Figure 7: AddCanonical $(p, r)$ 


\section{Chapter 4}

\section{$\mathrm{D} 8(\mathrm{P})$ is Degree 8}

\subsection{Introduction}

In this chapter we prove that the graph $D 8(P)$ has a maximum degree of 8 . We use a simple charging argument. Each edge $(p, q)$ of $D 8(P)$ is charged once to $p$ and once to $q$. Thus the total charge on a vertex is equal to the degree of that vertex. We show that no vertex is charged more than 8 .

In Section 4.2 we show that after the addition of the edges of $E_{A}$, the total charge on each vertex is at most 6 .

In Section 4.3 we show that by adding the edges of $E_{C A N}$, the total charge of each vertex does not exceed 8. Showing this is considerably more involved. To track the total number of charges on a vertex, we associate each charge with a cone. By limiting the charges to each cone, we can limit the total charge on a vertex, and thus the degree.

In Section 4.3.1 we characterize the cones into distinct types. In Section 4.3.2 we look at cones in cone neighbourhoods, and find cones that are unique to a neighbourhood. In Section 4.3.3 we look at where the possibility of cones that are shared between two neighbourhoods exists, and how we resolve this issue. In Section 4.3.4, we establish what cones are available to be charged by edges of $E_{C A N}$.

In Section 4.4 we provide the charging scheme, in terms of what cones of the endpoints are charged. In Section 4.5 we look at and resolve some ambiguous areas of the charging 
scheme, and prove the degree of $D 8(P)$.

\subsection{The Edges of $E_{A}$}

Lemma 4.2.1. An arbitrary vertex $p$ of the graph $D 8(P)$ is charged at most 6 by edges of $E_{A}$.

Proof. It follows from Step 2a in algorithm AddIncident(), that for each cone $C_{i}^{p}$, at most one edge $(p, q)$ is added to $E_{A}$ for each cone with apex $p$.

\subsection{The Edges of $E_{C A N}$}

\subsubsection{Cone Types}

Our charging argument hinges on which cones are charged and how many edges are charged in each one. We must therefore track the cones that have been charged. With this in mind we provide the following definitions:

Definition 4.3.1. For an arbitrary cone neighbourhood $N_{i}^{p}$ we define the region of $N_{i}^{p}$ as the polygonal region bounded by the canonical edges of $N_{i}^{p}$ and the first and last edge of $N_{i}^{p}$ with endpoint p. See Figure $8 a$.

Definition 4.3.2. Let $(a, b)$ be the first edge and let $(y, z)$ be the last edge in a canonical

subgraph $\operatorname{Can}_{i}^{(p, r)}$ (Definition 3.2.1). We define the region of $\operatorname{Can}_{i}^{(p, r)}$ as the polygonal region bounded by the canonical edges of $N_{i}^{p}$ between $(a, b)$ and $(y, z)$ inclusive, and the edges $(p, a)$ and $(p, z)$. See Figure $8 b$.

We provide the following definitions regarding the placement of cones in regions. Both of the following definitions also extend to regions of a cone neighbourhood. 


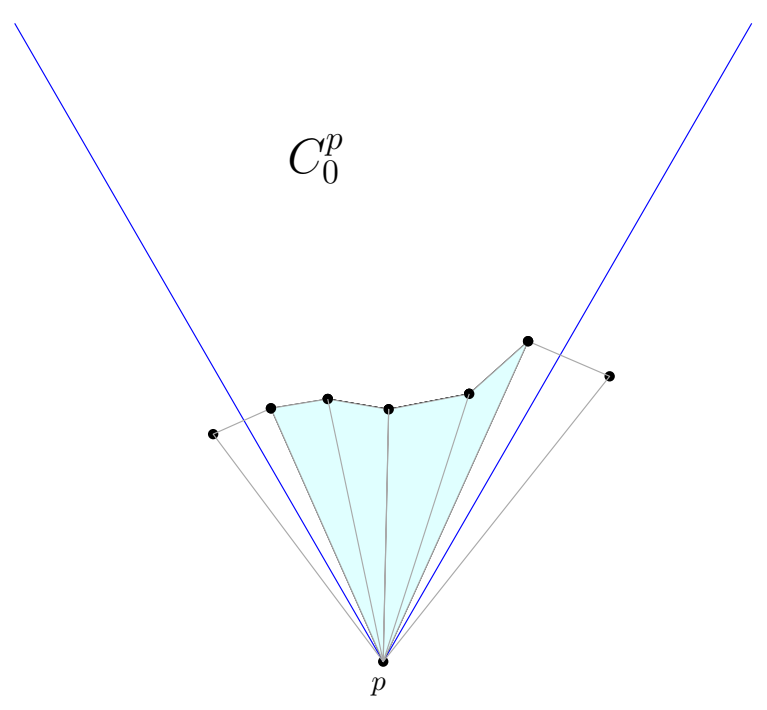

(a) The region of $N_{0}^{p}$ is light blue.

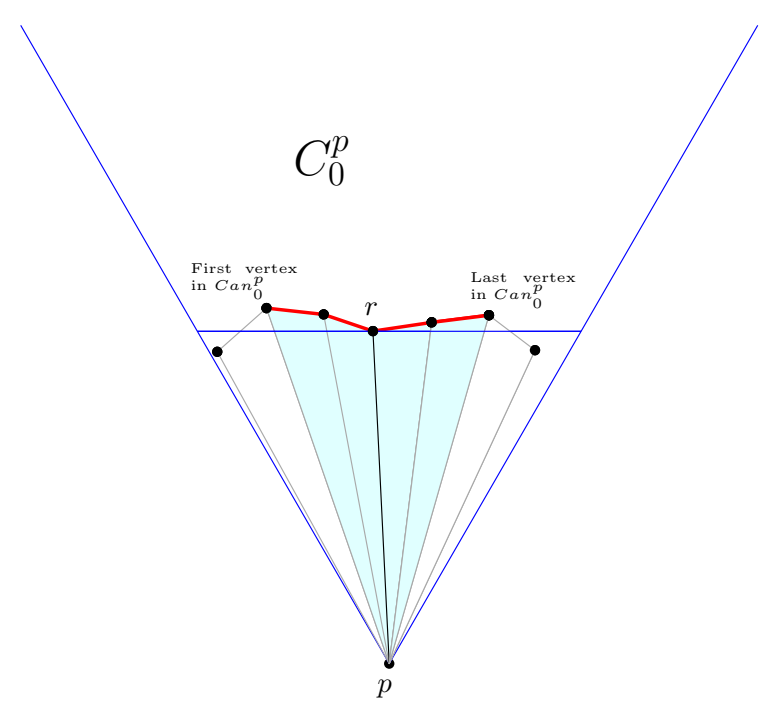

(b) The region of $\operatorname{Can}_{0}^{(p, r)}$ is light blue.

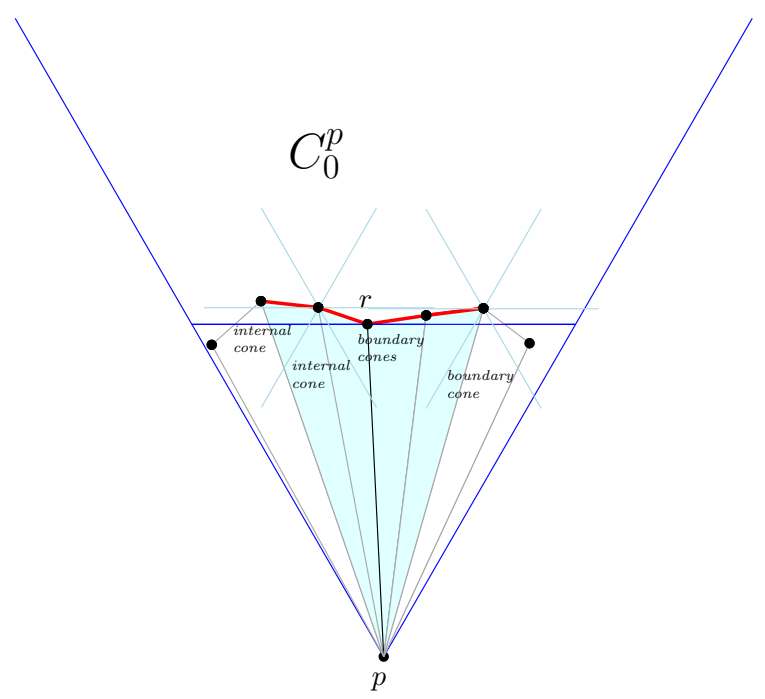

(c) Illustrating different types of cones on a canonical region.

Figure 8: Regions and cones. 
Definition 4.3.3. Let $B(s, \epsilon)$ be a ball with center $s$ and radius $\epsilon>0$. Consider a cone $C_{j}^{s}$ of a point s in $\mathrm{Can}_{i}^{(p, r)}$. If there exists an $\epsilon>0$ such that $B(s, \epsilon) \cap C_{j}^{s}$ is inside the region of $\mathrm{Can}_{i}^{(p, r)}$, then we call this an internal cone of $\mathrm{Can}_{i}^{(p, r)}$. Alternatively we say $C_{j}^{s}$ is in $\operatorname{Can}_{i}^{(p, r)}$.

Definition 4.3.4. Consider a cone $C_{j}^{s}$ of a point $s$ in $\operatorname{Can}_{i}^{(p, r)}$. If for all $\epsilon>0, B(s, \epsilon) \cap$ $C_{j}^{s}$ is partially but not entirely in the region of $\mathrm{Can}_{i}^{(p, r)}$, then we call this a boundary cone of $C a n_{i}^{(p, r)}$. Alternatively we say $C_{j}^{s}$ is on the boundary of $\mathrm{Can}_{i}^{(p, r)}$.

Definition 4.3.5. A cone with vertex $s$ as endpoint is empty if no edge of $E_{A}$ or $E_{C A N}$ incident to $s$ is in the cone.

In order to properly count the number of charges to a particular vertex, in this section we associate each charge of an edge to a particular cone of a vertex. By summing the charges to each cone surrounding a vertex, we are able to prove a maximum degree of 8 for any vertex of $D 8(P)$.

When referring to an angle formed by three points, we refer to the smaller of the two angles (that is, the angle that is $<\pi$ ) unless otherwise stated. When referring to a circle through three points $p_{1}, p_{2}$, and $p_{3}$, we use the notation $O_{p_{1}, p_{2}, p_{3}}$.

\subsubsection{Cones in Neighbourhoods}

We consider the edge $(p, r)$ of $E_{A}$, where without loss of generality, $r$ is in $C_{0}^{p}$. In this section we show the location of cones in the region of $\operatorname{Can}_{0}^{(p, r)}$, so we may charge edges of $E_{C A N}$ to them. To facilitate this we introduce another variation on the concept of the neighbourhood of a vertex:

Definition 4.3.6. Consider the cone neighbourhood $N_{i}^{p}$ with the vertex set $\left\{p, q_{0}, q_{1}, \ldots q_{m-1}\right\}$, where $\left\{q_{0}, q_{1}, \ldots q_{m-1}\right\}$ are listed in clockwise order around $p$. A restricted 
neighbourhood $N_{p}^{\left(q_{j}, q_{k}\right)}$ is the subgraph of $N_{i}^{p}$ induced on the vertex set $\left\{p, q_{j}, q_{j+1} \ldots, q_{k}\right\}, 0 \leq$ $j \leq k \leq m-1$.

Now we illustrate some of the geometric properties of restricted neighbourhoods in $D T(P)$.

Lemma 4.3.7. Consider the arbitrary restricted neighbourhood $N_{p}^{(r, q)}$. Each vertex $x \in$ $N_{p}^{(r, q)} \backslash\{p, r, q\}$ is in the circle $O_{p, r, q}$ through $p, r$, and $q$.

Proof. Since $(p, x)$ is an edge in $D T(P)$, we can draw a disk through $p$ and $x$ that is empty of points of $P$. In particular, neither $r$ nor $q$ is in this disk. Hence the sum of the angles $\angle(p r x)$ and $\angle(p q x)$ which lie on opposite sides of the same chord is smaller than $\pi$, and the sum of the other two angles $\angle(r x q)$ and $\angle(r p q)$ in the quadrilateral ( $p r x q)$ is greater then $\pi$. That implies $x$ is inside $O_{p, r, q}$.

Lemma 4.3.8. Consider a cone neighbourhood $N_{p}^{(r, q)}$ in cone $C_{i}^{p}$. Let $(p, x)$ be an edge in $N_{p}^{(r, q)}$ where $x \neq r$ and $x \neq q$. Then angle $\angle(q x r) \geq \pi-\angle(q p r)$. Since the cone angle is $\pi / 3$, we have that $\angle(q x r)>2 \pi / 3$.

Proof. We know by Lemma 4.3.7 that $x$ lies inside the circle through $p, r$ and $q$, which we label $O_{p, r, q}$. The angle $\angle(q x r)$ is minimized when $x$ is on $O_{p, r, q}$. When $x$ is on $O_{p, r, q}$, $\angle r x q=\pi-\angle(q p r)$, since the two angles lie on the same chord $(r, q)$. Therefore $\angle(r x q) \geq$ $\pi-\angle(q p r)$. Since both $q$ and $r$ are in the same cone $C_{i}^{p}$, and the cone angle is $\pi / 3$, the $\angle(q x r)>2 \pi / 3$.

Which leads to the corollary:

Corollary 4.3.9. Let $s$ be an inner vertex of $\operatorname{Can}_{i}^{(p, r)}$ that is not the anchor. Then there is at least one empty cone of $s$ in $\operatorname{Can}_{i}^{(p, r)}$. 


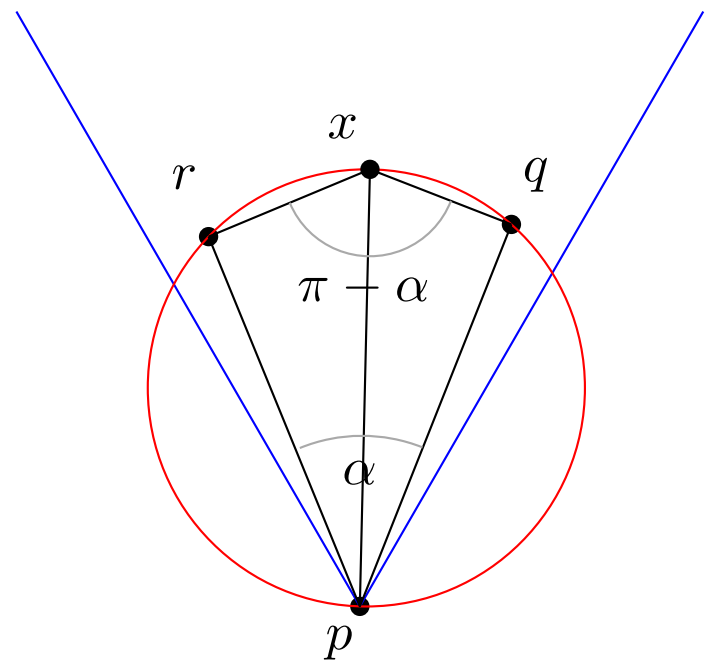

(a) When corners of a quadrilateral lie on a circle, opposite angles will sum to $\pi$.

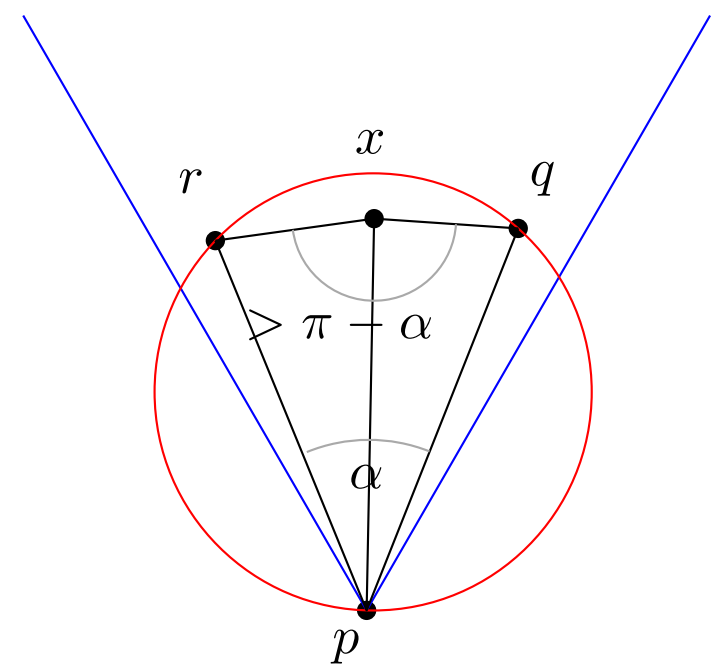

(b) When one corner of a quadrilateral lies inside a circle, that corner and its opposite angles will sum to $>\pi$.

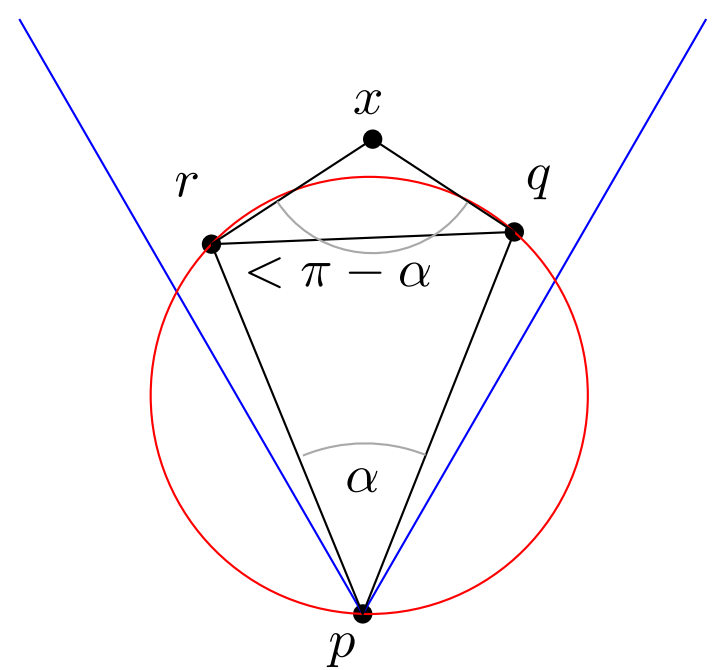

(c) When one corner of a quadrilateral lies outside a circle, that corner and its opposite angle will sum to $<\pi$.

Figure 9: Properties of convex quadrilaterals in $D T(P)$. 


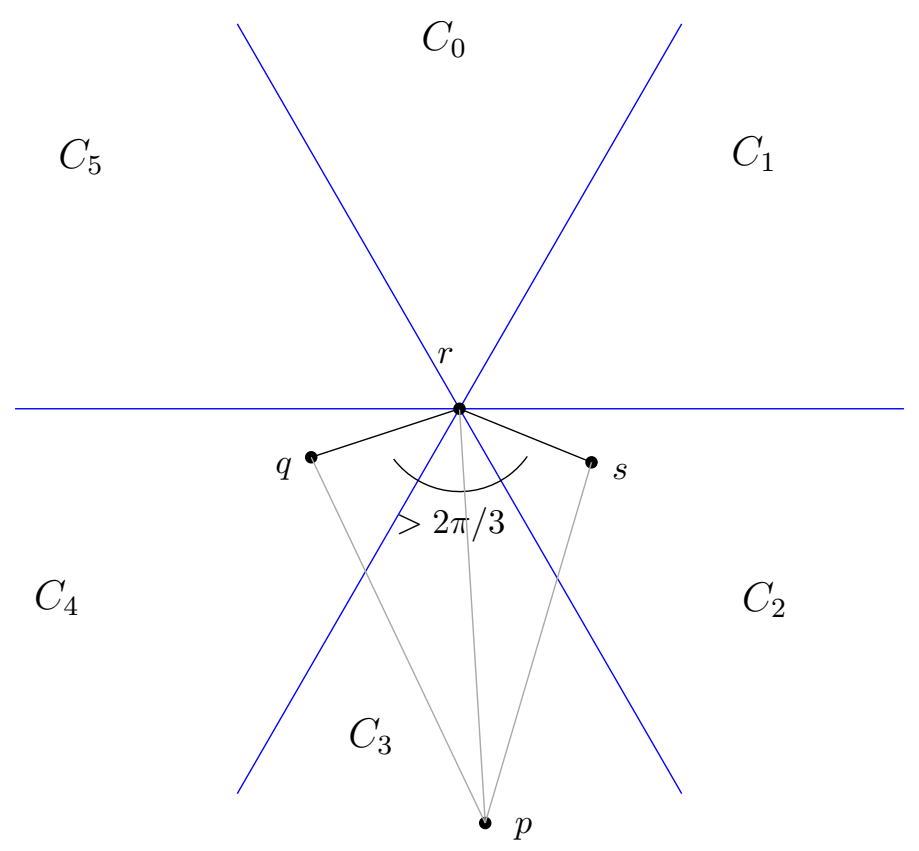

Figure 10: Consecutive canonical edges have an angle facing $p$ of at least $2 \pi / 3$, and thus if $(p, r) \notin$ $E_{A}$, there is at least one empty cone between them in $D 8(P)$.

Proof. Since $s$ is not the anchor, any internal cone of $\operatorname{Can}_{i}^{(p, r)}$ on vertex $s$ is empty, and by Lemma 4.3.8, there is at least one internal cone of $\operatorname{Can}_{i}^{(p, r)}$ on vertex $s$. Therefore there is at least one empty internal cone on $s$ in the region of $\operatorname{Can}_{i}^{(p, r)}$. See Figure 10.

Lemma 4.3.10. Consider the edge $(p, r)$ in $E_{A}$, and without loss of generality let $r$ be in $C_{0}^{p}$. If $r$ is an inner anchor of $C a n_{i}^{(p, r)}$, then cones $C_{2}^{r}$ and $C_{4}^{r}$ are empty and in the region of $\mathrm{Can}_{i}^{(p, r)}$.

Proof. Since $(p, r)$ is in $C_{0}^{p}$, it must also be in $C_{3}^{r}$, and thus it is in neither $C_{2}^{r}$ or $C_{4}^{r}$.

Assume that $q, r$ and $s$ are in consecutive order in $\operatorname{Can}_{i}^{(p, r)}$. Thus $\operatorname{Can}_{i}^{(p, r)}$ contains canonical edges $(q, r)$ and $(r, s)$.

Recall that for every vertex $x$ in $\operatorname{Can}_{i}^{(p, r)},[p x] \geq[p r]$. Thus $[p s] \geq[p r]$ and $[p q] \geq[p r]$, which means that $s$ and $q$ are above the horizontal line through $r$ in $C_{0}^{p}$. Since $C_{2}^{r}$ and $C_{4}^{r}$ lie below the horizontal line through $r$, they cannot contain the edges $(q, r)$ and $(r, s)$. Since 


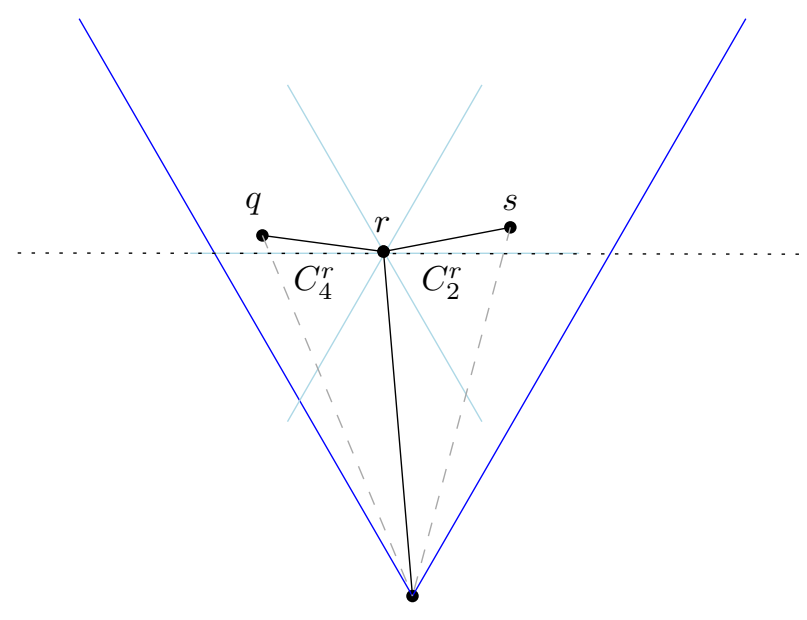

Figure 11: Lemma 4.3.10

$\triangle(p q r)$ and $\triangle(p r s)$ are triangles in $D T(P), C_{2}^{r}$ and $C_{4}^{r}$ are empty and inside $\operatorname{Can}_{i}^{(p, r)}$. See Figure 11

\subsubsection{Cones in Shared Triangles}

We will show the location of uncharged cones in the special case of overlapping regions. A set of regions overlap when at least one triangle of $D T(P)$ is contained in the intersection of all the regions in the set.

Lemma 4.3.11. Consider the triangle $\triangle\left(p p^{\prime} s\right)$ in $D T(P)$. Let $p^{\prime}$ and $s$ be in $C_{0}^{p}$, and let $p$ and $s$ be in $C_{3}^{p^{\prime}}$. Then $\angle\left(p^{\prime} s p\right)>2 \pi / 3$.

Proof. Without loss of generality, assume that $s$ is left of directed line segment $\left(p, p^{\prime}\right)$. Consider the parallelogram formed by $C_{0}^{p} \cap C_{3}^{p^{\prime}}$. Let $a$ be the left intersection and $b$ be the right intersection of $C_{0}^{p}$ and $C_{3}^{p^{\prime}}$. Thus $s$ is in $\triangle\left(a p p^{\prime}\right)$. Note that $\angle\left(p p^{\prime} a\right)+\angle\left(a p p^{\prime}\right)=\pi / 3$. Thus $\angle\left(p p^{\prime} s\right)+\angle\left(s p p^{\prime}\right)<\pi / 3$, which implies that $\angle\left(p^{\prime} s p\right)>2 \pi / 3$. See Figure 12 .

Lemma 4.3.12. Let $\triangle\left(p p^{\prime} s\right)$ be a triangle in $D T(P)$. Then $\triangle\left(p p^{\prime} s\right)$ can belong to cone neighbourhoods of at most two of $p, p^{\prime}$ and $s$. 


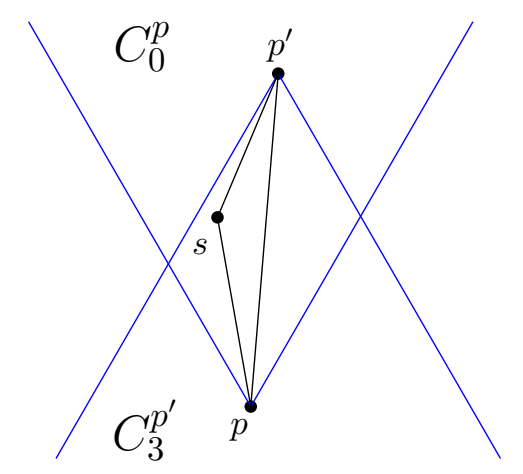

Figure 12: A triangle shared by $N_{0}^{p}$ and $N_{3}^{p^{\prime}}$.

Proof. Suppose, for the sake of contradiction, that triangle $\triangle\left(p p^{\prime} s\right)$ is in a cone neighbourhood of $p$, and a cone neighbourhood of $p^{\prime}$, and a cone neighbourhood of $s$. Without loss of generality, let $p^{\prime}$ and $s$ be in $C_{0}^{p}$. This means that $p^{\prime}$ and $s$ must be in $C_{3}^{p^{\prime}}$. By Lemma 4.3.11 angle $\angle\left(p s p^{\prime}\right)>2 \pi / 3$. Therefore $p$ and $p^{\prime}$ cannot be in the same cone neighbourhood of $s$.

Corollary 4.3.13. A triangle can be shared by at most 2 cone neighbourhoods.

Proof. Follows from Lemma 4.3.12.

Which leads to the following definition:

Definition 4.3.14. If $\triangle\left(p p^{\prime} s\right)$ occurs in exactly two cone neighbourhoods of $p, p^{\prime}$ and $s$, then we refer to it as a shared triangle. If $\triangle\left(p p^{\prime} s\right)$ is in cone neighbourhoods of $p$ and $p^{\prime}$, then $\left(p, p^{\prime}\right)$ is referred to as the base of the shared triangle.

Corollary 4.3.15. In a shared triangle $\triangle\left(p p^{\prime} s\right)$ with base $\left(p, p^{\prime}\right)$, s has two empty cones internal to $\triangle\left(p p^{\prime} s\right)$.

Proof. Without loss of generality, assume that $p^{\prime} \in C_{0}^{p}$ and $p \in C_{3}^{p^{\prime}}$. Then $p \in C_{3}^{s}$ and $p^{\prime} \in C_{0}^{s}$. Then either $C_{2}^{s}$ and $C_{1}^{s}$, or $C_{5}^{s}$ and $C_{4}^{s}$ are internal to $\triangle\left(p p^{\prime} s\right)$ and thus cannot 


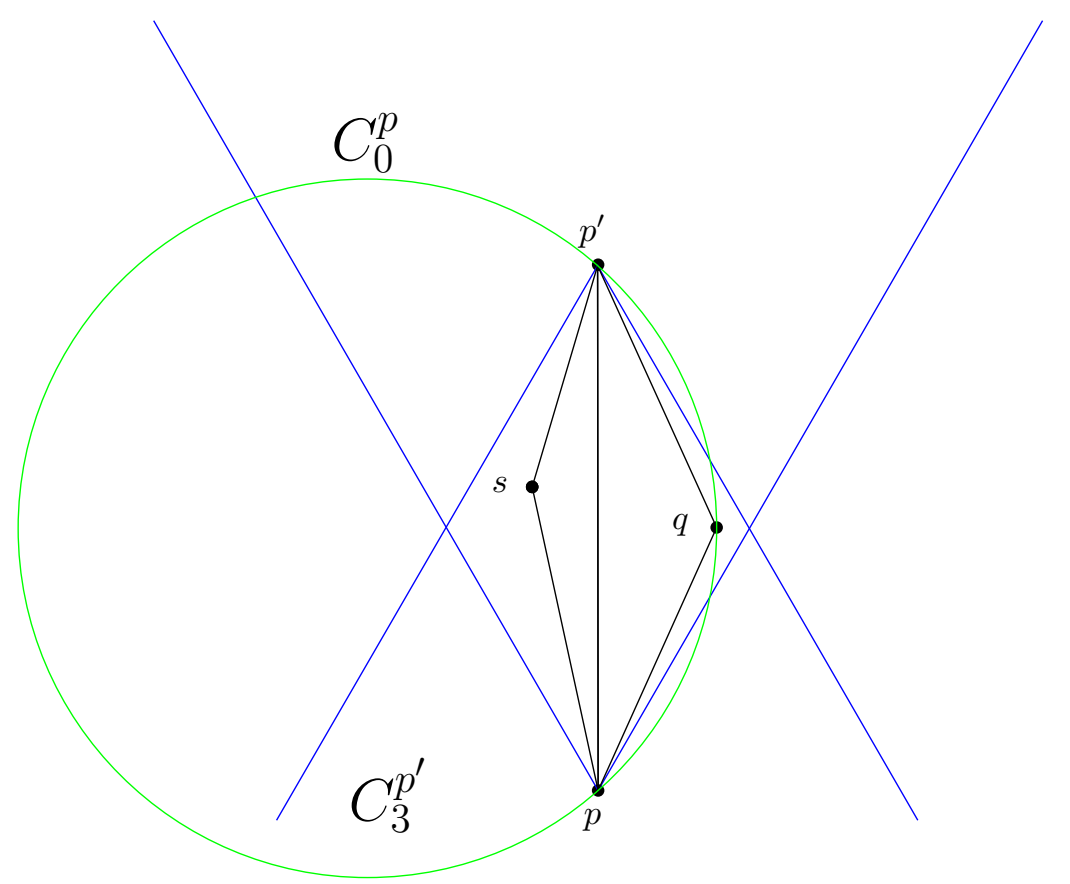

Figure 13: $q \in C_{0}^{p} \cap C_{3}^{p^{\prime}}$ violates the empty circle property of Delaunay triangulations.

contain any edges with endpoint $s$.

We show two cone neighbourhoods can share at most one triangle:

Lemma 4.3.16. Each Delaunay edge is the base of at most 1 shared triangle.

Proof. By contradiction.

Consider the shared triangle $\triangle\left(p p^{\prime} s\right)$ with base $\left(p, p^{\prime}\right)$. Without loss of generality, assume $p^{\prime}$ is in $C_{0}^{p}$, and $p$ is in $C_{3}^{p^{\prime}}$. Since $\left(p, p^{\prime}\right)$ is an edge in exactly two triangles, let $\triangle\left(p p^{\prime} q\right)$ be the other triangle with edge $\left(p, p^{\prime}\right)$, and assume that $q$ is in both $C_{0}^{p}$ and $C_{3}^{p^{\prime}}$.

By Lemma 4.3.11 both angles $\angle\left(p^{\prime} s p\right)$ and $\angle\left(p q p^{\prime}\right)$ are greater than $2 \pi / 3$. Thus their sum is greater than $4 \pi / 3$. But by the empty circle property of Delaunay triangulations, $\angle\left(p^{\prime} s p\right)+\angle\left(p q p^{\prime}\right)$ must be less than $\pi$, which is a contradiction.

Corollary 4.3.17. Two cone neighbourhoods can share at most one triangle. 


\subsubsection{Empty Cones}

Lemma 4.3.18. Consider the edge $(p, r)$ in $E_{A}$. Without loss of generality let $r$ be in $C_{0}^{p}$. Each inner vertex $s$ of $\operatorname{Can}_{0}^{(p, r)}$ that is not the anchor has at least one unique empty cone in the region of $\mathrm{Can}_{0}^{(p, r)}$.

Proof. If $s$ is not part of a shared triangle, we know by Corollary 4.3 .9 that $s$ has an empty cone internal to $\operatorname{Can}_{0}^{(p, r)}$.

Consider the shared triangle $\triangle\left(p p^{\prime} s\right)$, and without loss of generality, let $p^{\prime}$ be in $C_{0}^{p}$. Assume that there is an edge $(p, r)$ of $E_{A}$ in $C_{0}^{p}$, and an edge $\left(p^{\prime}, r^{\prime}\right)$ of $E_{A}$ in $C_{3}^{p^{\prime}}$. Thus both sets $\operatorname{Can}_{0}^{(p, r)}$ and $\operatorname{Can}_{3}^{\left(p^{\prime}, r^{\prime}\right)}$ are well-defined.

By Corollary 4.3.15 there are two empty cones of $s$ internal to $\triangle\left(p p^{\prime} s\right)$. The empty cone adjacent to $\left(p^{\prime}, s\right)$ is the empty cone of $s$ in the region of $\operatorname{Can}_{0}^{(p, r)}$, and the empty cone adjacent to $(p, s)$ is the empty cone of $s$ in the region of $\operatorname{Can}_{3}^{\left(p^{\prime}, r^{\prime}\right)}$.

Thus any inner vertex $s$ of an arbitrary canonical subgraph $\operatorname{Can}_{0}^{(p, r)}$ that is not the anchor, has a unique empty cone that is in the region of $\operatorname{Can}_{0}^{(p, r)}$.

Lemma 4.3.19. Consider the edge $(p, r)$ in $E_{A}$. Without loss of generality let $r$ be in $C_{0}^{p}$. Let $z \neq r$ be an end vertex in $\operatorname{Can}_{0}^{(p, r)}$. By symmetry, let $z$ be the last vertex. Let $y$ be the neighbour of $z$ in $C a n_{0}^{(p, r)}$. If $y$ is in $C_{5}^{z}$, then $C_{4}^{z}$ is an empty cone internal to the region of $\operatorname{Can}_{0}^{(p, r)}$.

Proof. Triangle $\triangle(p y z)$ is a triangle in $D T(P)$. Since $(p, z)$ is in $C_{3}^{z}$ and $(y, z)$ is in $C_{5}^{z}, C_{4}^{z}$ will have no edges in $D T(P)$ with endpoint $z$. Since both $E_{A}$ and $E_{C A N}$ are subsets of the edges of $D T(P), C_{4}^{z}$ will not contain any edges of $E_{A}$ and $E_{C A N}$ with endpoint $s$, and thus is empty. 


\subsection{Charging Scheme for D8(P)}

Recall that $D 8(P)$ consists of the point set $P$ and the edge set $E_{A} \cup E_{C A N}$.

We present the charging scheme that will allow us to prove that $D 8(P)$ has a maximum degree 8. Each edge charges one cone for each endpoint. Therefore the total charge on the cones of a vertex will be exactly the degree of the vertex. We will show that no vertex will be charged more than 8 .

We begin with the charging scheme for the edges of $E_{A}$. Consider an edge $(p, r)$ of $E_{A}$, where without loss of generality $r$ is in $C_{0}^{p}$ and $p$ is in $C_{3}^{r}$.

$E_{A}$ Charge 1: An edge $(p, r)$ of $E_{A}$ charges $C_{0}^{p}$ once and $C_{3}^{r}$ once.

We now show to which cones the endpoints of the edges in $E_{C A N}$ are charged. Consider an arbitrary cone neighbourhood $N_{i}^{p}$, and without loss of generality let $i=0$. We consider a call to $\operatorname{AddCanonical}(p, r)$, where $(p, r)$ is an edge in $E_{A}$. Without loss of generality, let $r$ be in $C_{0}^{p}$. By outlining the algorithm $\operatorname{AddCanonical}(p, r)$ in its entirety, and for each step of $\operatorname{AddCanonical}(p, r)$ that adds edges to $E_{C A N}$, we describe which cones of the endpoints an edge is charged to.

Without loss of generality, let $(p, r)$ be an edge of $E_{A}$, where $r \in C_{0}^{p}$. Let $\operatorname{Can}_{0}^{(p, r)}$ be the subgraph consisting of the ordered subsequence of canonical edges $(s, t)$ of $N_{0}^{p}$ in clockwise order around apex $p$ such that $[p s] \geq[p r]$ and $[p t] \geq[p r]$. We call $\operatorname{Can}_{0}^{(p, r)}$ a canonical subgraph.

Step 1: If the anchor $r$ is the first or last vertex in $\operatorname{Can}_{0}^{(p, r)}$, and there is more than one edge in $C a n_{0}^{(p, r)}$, then add the edge of $\operatorname{Can}_{0}^{(p, r)}$ with endpoint $r$ to $E_{C A N}$. See Figure $7 \mathrm{~b}$.

Step 2: If there are at least three edges in $\mathrm{Can}_{0}^{(p, r)}$, then for every canonical edge $(s, t)$ in $\mathrm{Can}_{0}^{(p, r)}$ that is not the first or last edge in the ordered subsequence of canonical edges $C a n_{0}^{(p, r)}$, we add $(s, t)$ to $E_{C A N}$.

The edge $(s, t)$ is charged once to $s$ and once to $t$. Since the charging scheme is the same for both $s$ and $t$, without loss of generality we only describe how to charge $s$. 


\section{Charge vertex $s$ :}

(a) If $s$ is the anchor (thus $s=r$ ), then by Lemma 4.3.10, $C_{2}^{r}$ and $C_{4}^{r}$ are empty cones inside $\operatorname{Can}_{0}^{(p, r)}$. If $t$ is left of directed line segment $p r$, charge $(r, t)$ to $C_{4}^{r}$. If $t$ is right of $p r$, charge $(r, t)$ to $C_{2}^{r}$. See Figure 18 .

(b) If $s \neq r$ then by Lemma 4.3.18, $s$ has an empty cone $C_{j}^{s}$ inside $\operatorname{Can}_{0}^{(p, r)}$. Charge $(s, t)$ once to $C_{j}^{s}$. See Figures 15, 16, 17.

Step 3: Consider the first and last canonical edge in $\mathrm{Can}_{0}^{(p, r)}$. Since the conditions for the first and last canonical edge are symmetric, we only describe how to process the last canonical edge $(y, z)$. There are three possibilities.

(a) If $(y, z) \in C_{5}^{z}$, add $(y, z)$ to $E_{C A N}$. See Figure $7 c$.

(b) If $(y, z) \in N_{4}^{z}$ and $N_{4}^{z}$ does not have an edge with endpoint $z$ in $E_{A}$, then we add $(y, z)$ to $E_{C A N}$. See Figure $7 d$

\section{Charge vertex $y$ :}

i If $y$ is the anchor, then $C_{2}^{y}$ is empty and inside $\operatorname{Can}_{0}^{(p, r)}$ by Lemma 4.3 .10 Charge $(y, z)$ to $C_{2}^{y}$. Figure 18 .

ii Otherwise $y$ is not the first or last vertex in $\operatorname{Can}_{0}^{(p, r)}$, and by Corollary 4.3.9 has an empty cone $C_{j}^{y}$ inside $\operatorname{Can}_{0}^{(p, r)}$. Charge $(y, z)$ to $C_{j}^{y}$. Figures $15,16$. 17.

\section{Charge vertex $z$ :}

iii Step 4a. $(y, z)$ is in $C_{5}^{z}$. By Lemma 4.3.19 $C_{4}^{z}$ is empty and inside $\operatorname{Can}_{0}^{(p, r)}$. Charge $(y, z)$ to $C_{4}^{z}$. Figure 20 .

iv Step 4b: $(y, z)$ is in $C_{4}^{z}$, and $C_{4}^{z}$ does not contain an edge of $E_{A}$ with endpoint $z$. Note $C_{4}^{z}$ is a boundary cone of $\operatorname{Can}_{0}^{(p, r)}$. Charge $(y, z)$ to $C_{4}^{z}$. Figure 19 . 
(c) If $(y, z) \in C_{4}^{z}$ and there is an edge $(u, z), u \neq y$ of $E_{A}$ in $C_{4}^{z}$, then there is one canonical edge of $z$ with endpoint $y$ in $C_{4}^{z}$. Label the edge $(w, y)$ and add it to $E_{C A N}$. See Figure 7 .

\section{Charge vertex $y$ :}

i If $y=r$, then $C_{2}^{y}$ is empty and inside $\operatorname{Can}_{0}^{(p, r)}$ by Lemma 4.3.10. Charge $(w, y)$ to $C_{2}^{y}$. Figure 18 .

ii Otherwise $y$ is not the first or last vertex in $\operatorname{Can}_{0}^{(p, r)}$, and by Corollary 4.3 .9 has an empty cone $C_{j}^{y}$ internal to $\operatorname{Can}_{0}^{(p, r)}$. Charge $(w, y)$ to $C_{j}^{y}$. Figure 21.

\section{Charge vertex $w$ :}

iii If $w=u\left((z, u)\right.$ in $\left.E_{A}\right)$, then $C_{2}^{w}$ is empty and inside $C a n_{4}^{(z, u)}$ by Lemma 4.4.1. Charge $(w, y)$ to $C_{2}^{w}$. Figure 18 .

iv If $w \neq u$, then $w$ is not the first or last vertex in $\operatorname{Can}_{4}^{(z, u)}$, and by Corollary 4.3.9 has an empty cone $C_{j}^{w}$ inside $\operatorname{Can}_{4}^{(z, u)}$. Charge $(w, y)$ to $C_{j}^{w}$. Figure 22

Step 3||c)||ii makes use of the following lemma:

Lemma 4.4.1. Assume that on a call to AddCanonical $(p, r)$, where $(p, r)$ is in $C_{0}^{p}$, we add edge $(w, y)$ to $E_{C A N}$ in Step 4 . Let $(y, z)$ be the last edge in $C a n_{0}^{(p, r)}$, and assume that $(w, z)$ is in $E_{A}$. Then $C_{2}^{w}$ is empty and inside $\operatorname{Can}_{4}^{(z, u)}$.

Proof. To prove this we shall establish that $[y z] \geq[w z]$. This, together with Lemma 4.3.10 implies that $C_{2}^{w}$ is empty and inside $\operatorname{Can}_{4}^{(z, u)}$.

We prove by contradiction, thus assume that $[w z]>[y z]$. See Figure 14 . This means that AddIncident $(L)$ examined $(y, z)$ before $(w, z)$, and thus $C_{4}^{z} \cap E_{A}$ was empty of edges with endpoint $z$ when $(y, z)$ was examined by $\operatorname{AddIncident}(L)$. Since $(y, z)$ was not added to $E_{A}, y$ must have had an edge of $E_{A}$ in $C_{1}^{y}$ with endpoint $y$ that was shorter than $(y, z)$. 

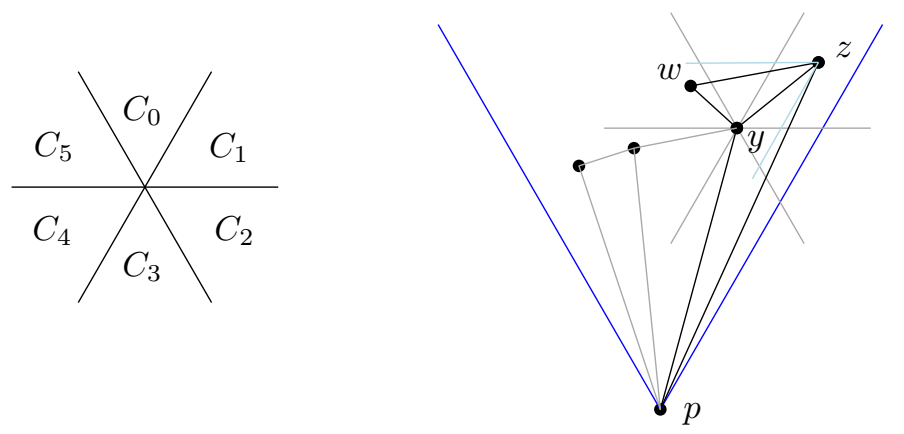

Figure 14: The case if $[w z]>[y z]$

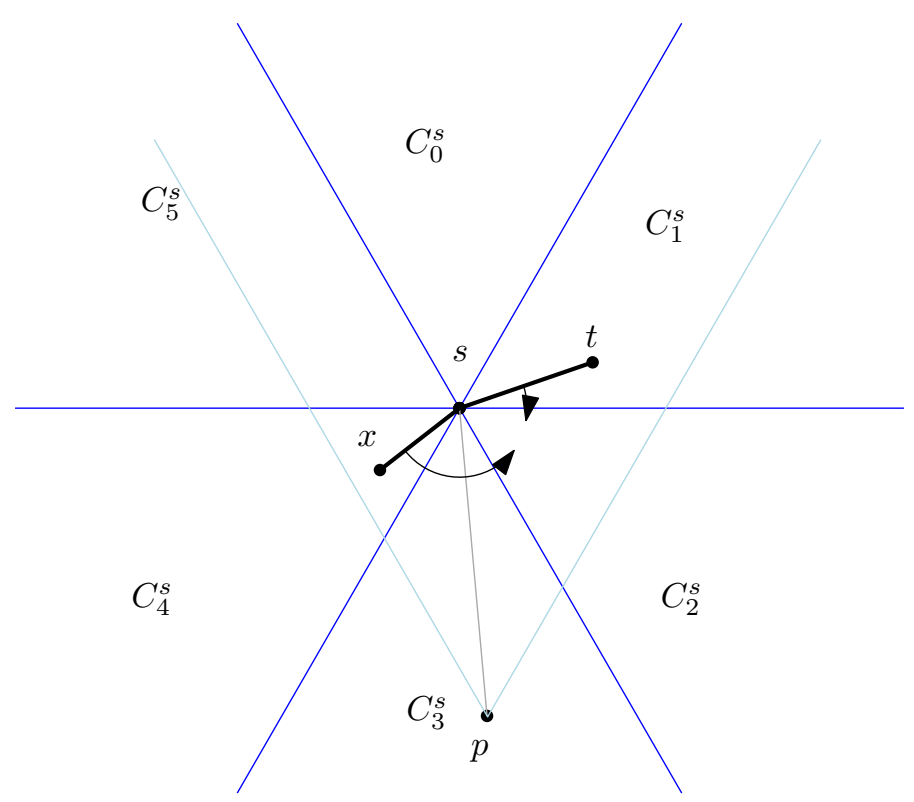

Figure 15: Vertex $s$ is an inner vertex of $\operatorname{Can}_{0}^{(p, r)}, s \neq r$. Charge $C_{2}^{s}$.

Since $\triangle(p y z)$ is a triangle in $D T(P)$, and $p \in C_{3}^{y}$, there cannot be an edge with endpoint $y$ in $C_{1}^{y}$ clockwise from $(y, z)$. In the counter-clockwise direction from $(y, z)$, we have the $\triangle(y w z) \in D T(P)$. However, since $[w z]>[y z],(w, y)$ cannot be in $C_{1}^{y}$. Thus $C_{1}^{y}$ contained no edge of $E_{A}$ with endpoint $y$ when $(y, z)$ was examined by $\operatorname{AddIncident}(L)$. Which means if $[w z]>[y z]$, then $(y, z)$ would have be added to $E_{A}$ by $\operatorname{AddIncident}(L)$. But we know $(z, w)$ is in $E_{A}$, therefore it must be that $[y z] \geq[w z]$, which implies that $C_{2}^{w}$ is empty and by Lemma 4.3 .10 is inside $\operatorname{Can}_{4}^{(z, u)}$. See Figure 14 . 


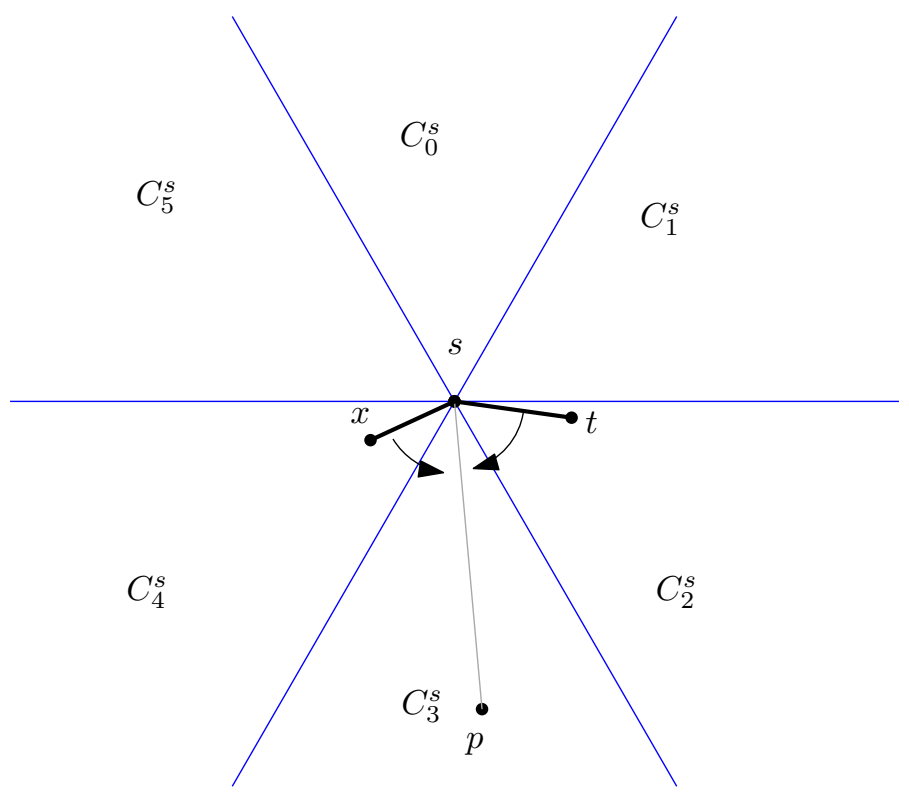

Figure 16: Vertex $s$ is neither the first nor last vertex of $\operatorname{Can}_{0}^{(p, r)}, s \neq r$.

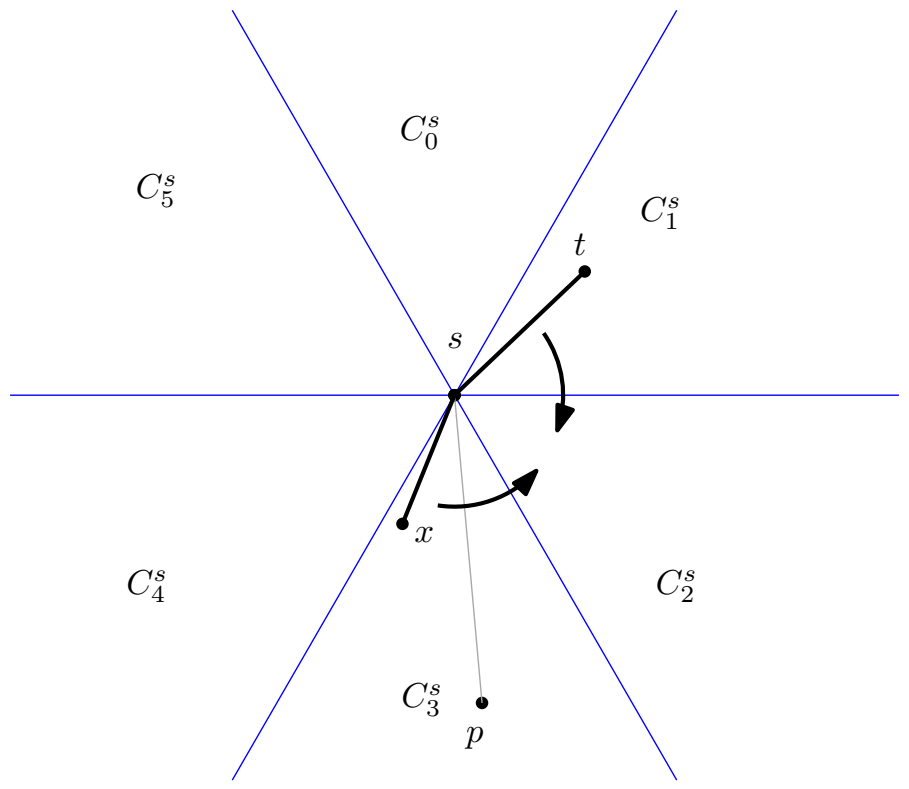

Figure 17: Vertex $s$ is neither the first nor last vertex of $\operatorname{Can}_{0}^{(p, r)}, s \neq r$. 


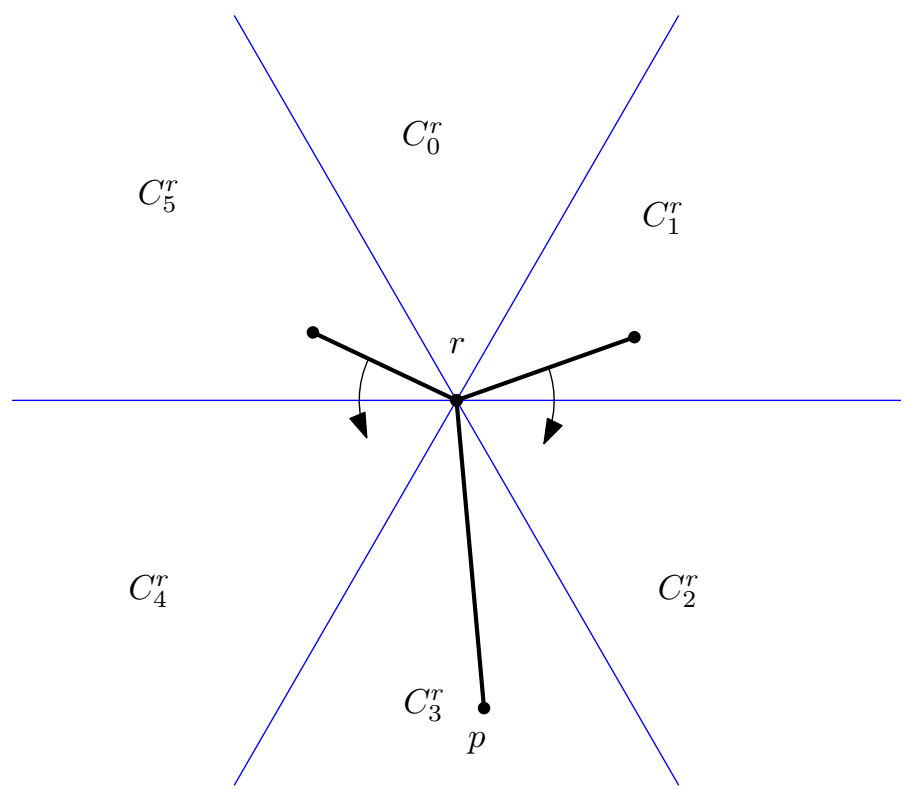

Figure 18: $(p, r) \in E_{A}$, canonical edges with endpoint $r$.

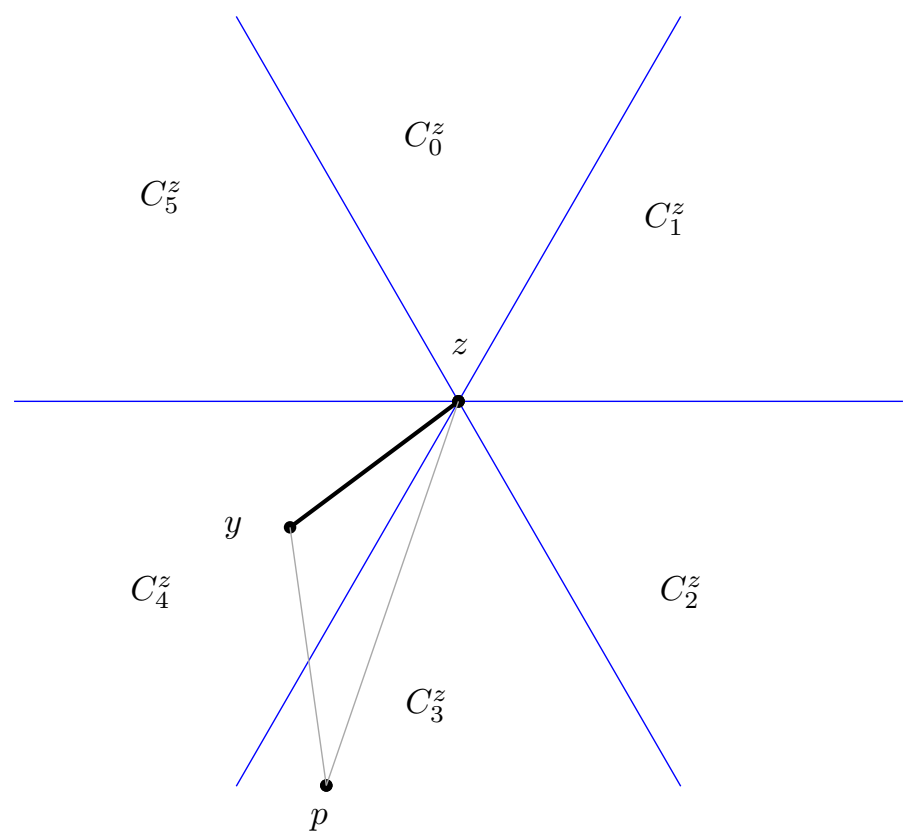

Figure 19: $(y, z)$ is the last canonical edge in $\operatorname{Can}_{0}^{(p, r)}$, and is charged to $C_{4}^{z}$. 


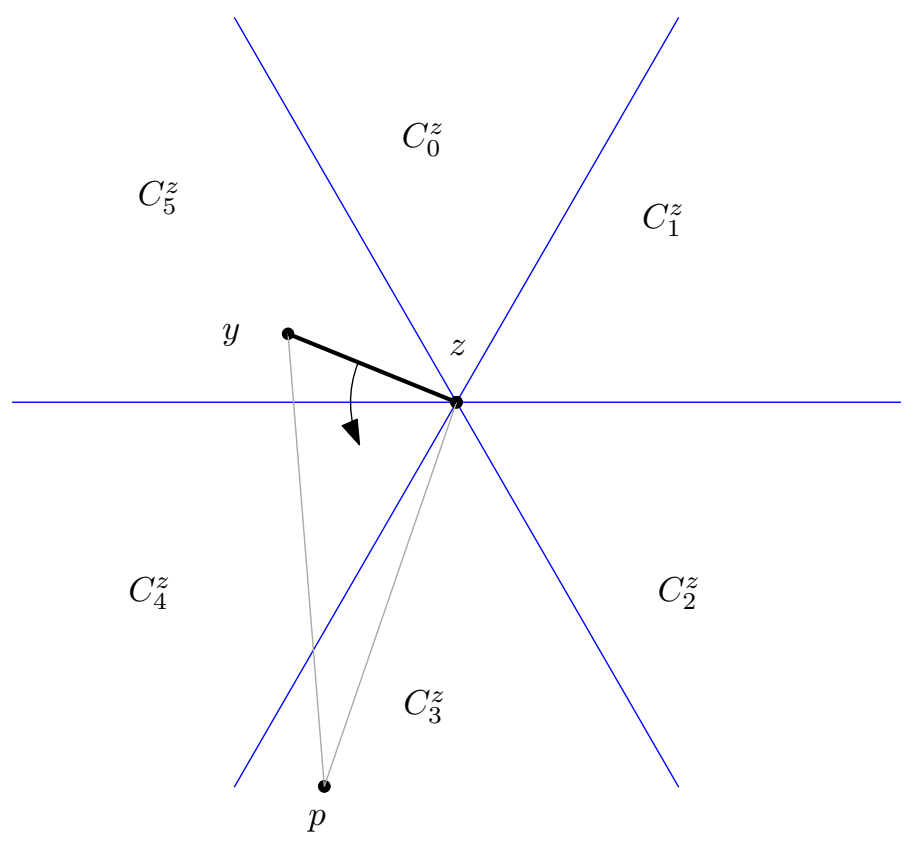

Figure 20: $(y, z)$ is the last canonical edge in $\operatorname{Can}_{0}^{(p, r)}$, and is charged to $C_{4}^{z}$.

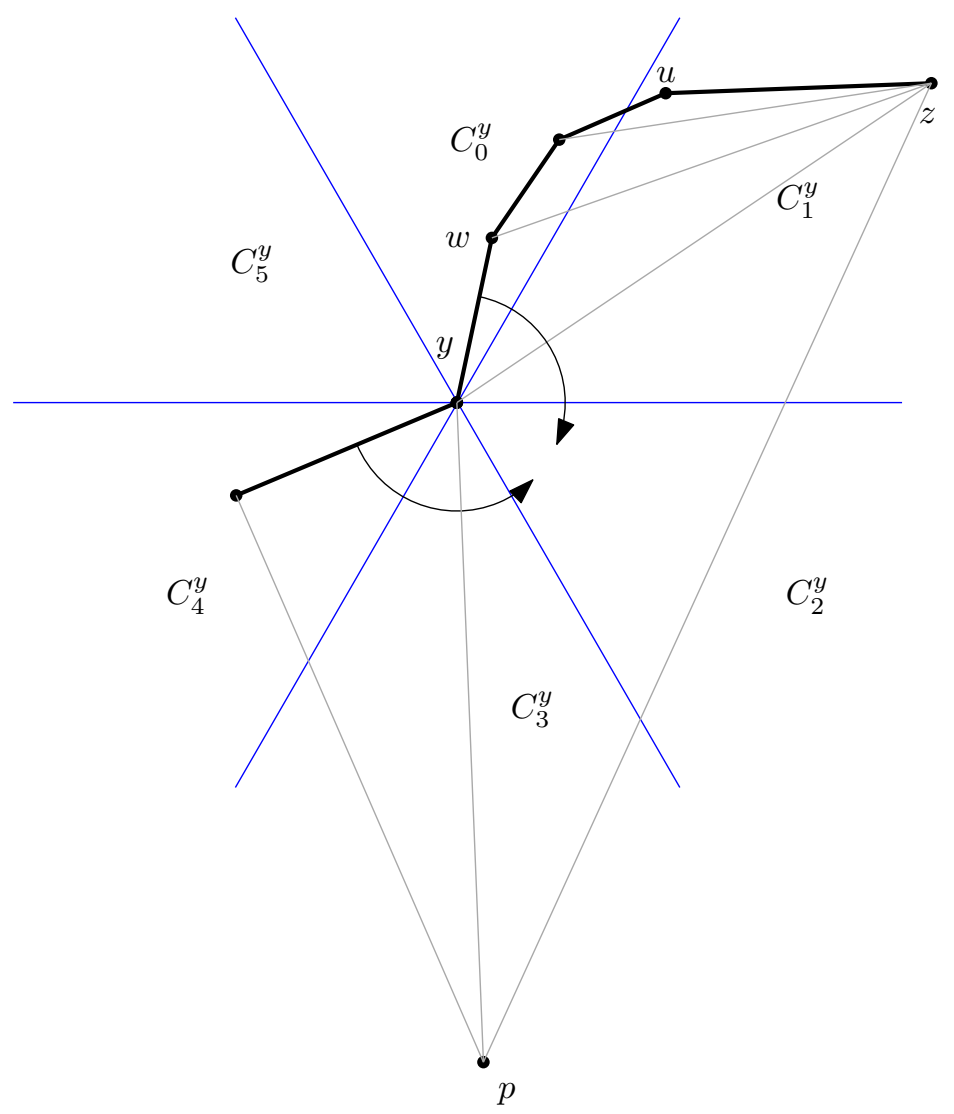

Figure 21: $(w, y)$ is charged to $y$ in place of $(y, z)$. 


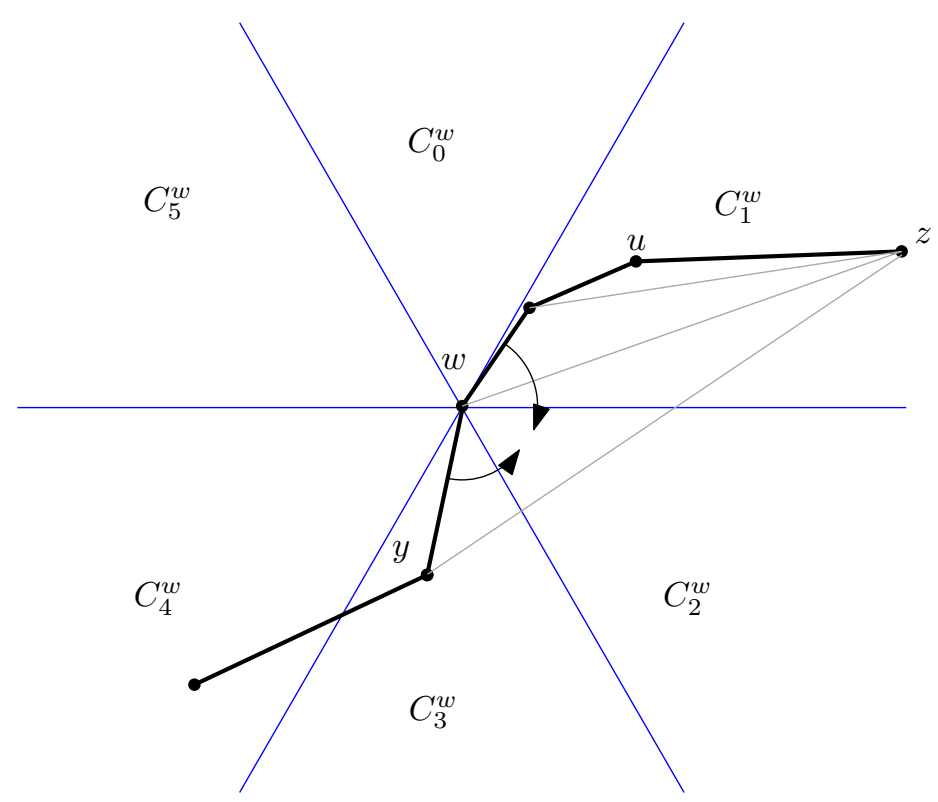

Figure 22: $(w, y)$ is charged to the empty cone of $w$.

\subsection{Proving the Degree of D8(P)}

\subsubsection{Preliminaries}

The charging argument of the previous section establishes where charges are made. In this section we show a limit to how many edges can be charged to the different cones.

\subsubsection{Edges in Boundary Cones}

All edges added to $E_{C A N}$ are charged to internal cones, with the exception of the edge that is added in $\operatorname{AddCanonical}(p, \cdot)$ Step $4 \mathrm{~b}$ to $C_{4}^{z}$, which is to a boundary cone. Since $C_{4}^{z}$ is on the boundary of $\operatorname{Can}_{0}^{(p, r)}$, it may also be the boundary cone of a different cone neighbourhood.

Lemma 4.5.1. The boundary cone $C_{4}^{z}$ of $C a n_{0}^{(p, r)}$ charged in AddCanonical $(p, r)$ Step 46 cannot be the internal cone of a different canonical subgraph.

Proof. $C_{4}^{z}$ is inside a canonical subgraph, then $y$ must be the apex of said canonical subgraph. That implies that both shared neighbours of $z$ and $y$ must be in $C_{1}^{y}$. But shared neighbour 
$p$ is in $C_{3}^{y}$, thus $C_{4}^{z}$ cannot be inside a canonical subgraph .

This implies that $C_{4}^{z}$ may only be shared with a different canonical subgraph as a boundary cone. Thus the only other edge of $E_{C A N}$ that can be charged to $C_{4}^{z}$ must be added in some call to AddCanonical $(\cdot, \cdot)$ Step 4b. We prove here this is impossible.

Lemma 4.5.2. Consider the edge $(p, r)$ of $E_{A}$ in $C_{i}^{p}$, and without loss of generality, let $i=0$. Let $(y, z)$ be the last edge in $\operatorname{Can}_{0}^{(p, r)}$, and let $z$ be the last vertex in $\operatorname{Can}_{0}^{(p, r)}$. Assume that $(y, z)$ was added to $E_{C A N}$ in a call to AddCanonical $(p, r)$ Step 4 b, and thus by Charge $i v$ is charged to the cone $C_{4}^{z}$. Then $(y, z)$ is the only edge in $D 8(P)$ charged to $C_{4}^{z}$.

Proof. Edge $(y, z)$ is added to $E_{C A N}$ in a call to $\operatorname{AddCanonical}(p, r)$ Step 4b, only if there is no edge of $E_{A}$ in $C_{4}^{z}$. Thus $C_{4}^{z}$ is not charged by an edge of $E_{A}$ by $E_{A}$ Charge 1 .

Cone $C_{4}^{z}$ is a boundary cone of $\operatorname{Can}_{0}^{(p, r)}$. By Lemma 4.5.1. $C_{4}^{z}$ cannot be an internal cone of another canonical subgraph. Thus $C_{4}^{z}$ can only be a boundary cone of any canonical subgraph. Since AddCanonical $(\cdot, \cdot)$ Step $4 \mathrm{~b}$ is the only call that adds an edge to $E_{C A N}$ that is charged to a boundary cone, only another call to AddCanonical $(\cdot, \cdot)$ Step 4b can charge an additional edge to $C_{4}^{z}$.

Assume we have edges $(y, z)$ and $\left(y^{\prime}, z\right)$ in $\operatorname{Can}_{i}^{(p, r)}$ and $\operatorname{Can}_{j}^{\left(p^{\prime}, r^{\prime}\right)}$ respectively. Without loss of generality, assume that $z$ is the first vertex in $\operatorname{Can}_{j}^{\left(p^{\prime}, r^{\prime}\right)}$ and the last vertex in $\operatorname{Can}_{i}^{(p, r)}$, and assume $(y, z)$ and $\left(y^{\prime}, z\right)$ occupy the same cone $C_{k}^{z}$. For both $(y, z)$ and $\left(y^{\prime}, z\right)$ to be added in AddCanonical $(\cdot, \cdot)$ Step $4 \mathrm{~b}$, it must be that $k=i-2=j+2$. Without loss of generality let $i=0, k=4$ and $j=2$, and $z$ is in $C_{0}^{p}$ and $C_{2}^{p^{\prime}}$.

We know that both $\triangle(p y z)$ and $\triangle\left(p^{\prime} y^{\prime} z\right)$ are triangles in $D T(P)$. Since $(y, z) \in C_{1}^{y}$, and $(y, p) \in C_{3}^{y}$, there is no edge in $C_{1}^{y}$ clockwise from $(y, z)$. Symmetrically, there is no edge in $C_{1}^{y^{\prime}}$ counter-clockwise from $\left(y^{\prime}, z\right)$. Thus $y$ must have a neighbour closer than $z$ in $C_{1}^{y}$ counter-clockwise from $(y, z)$, and $y^{\prime}$ must have a neighbour closer than $z$ in $C_{1}^{y^{\prime}}$ clockwise from $\left(y^{\prime}, z\right)$. See Figure 23 .

We consider the shorter of $(y, z)$ and $\left(y^{\prime}, z\right)$, ties broken arbitrarily. Without loss of 
Cone Legend:
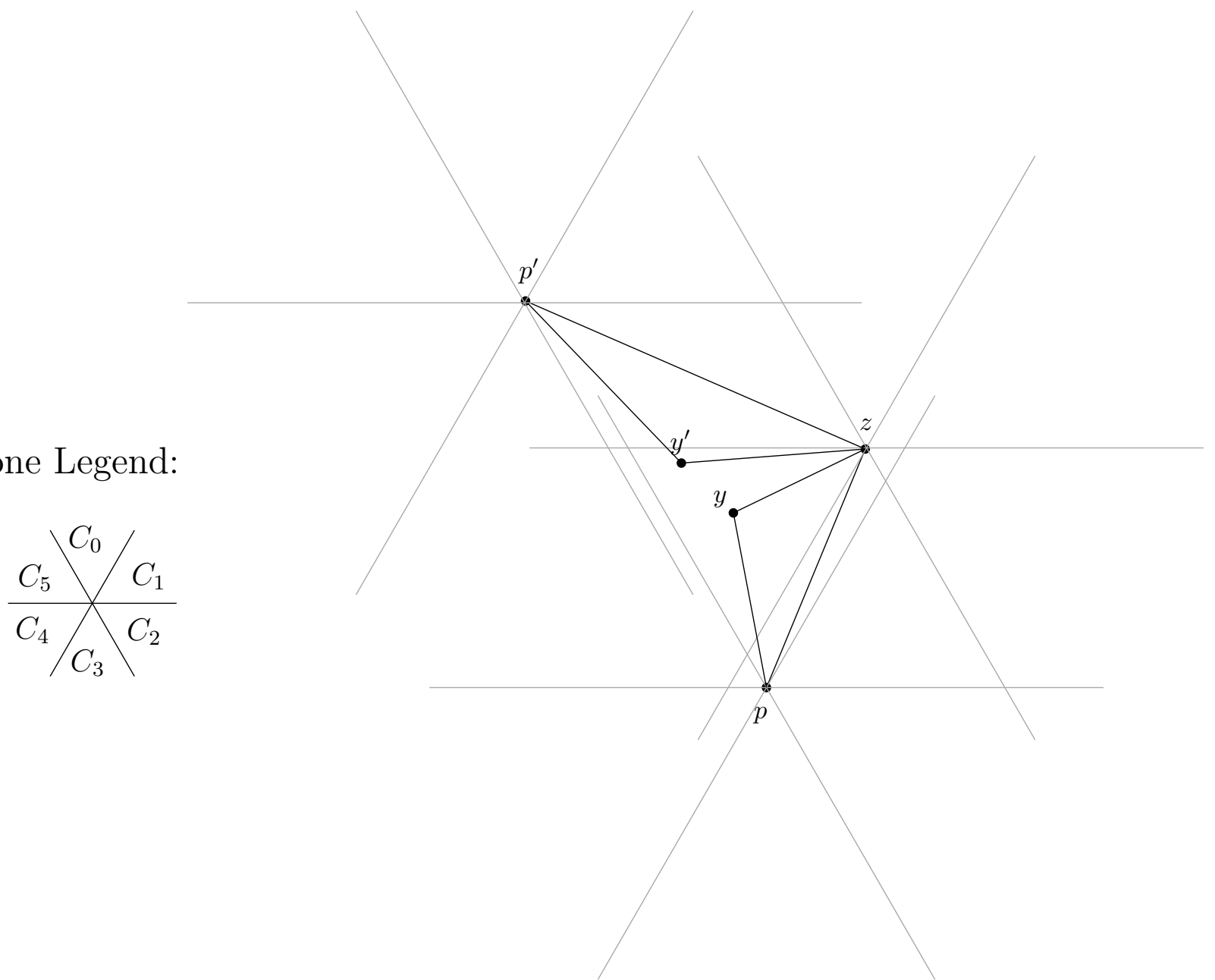

Figure 23: There must be an edge of $E_{A}$ between $(y, z)$ and $\left(y^{\prime}, z\right)$. 
Cone Legend:
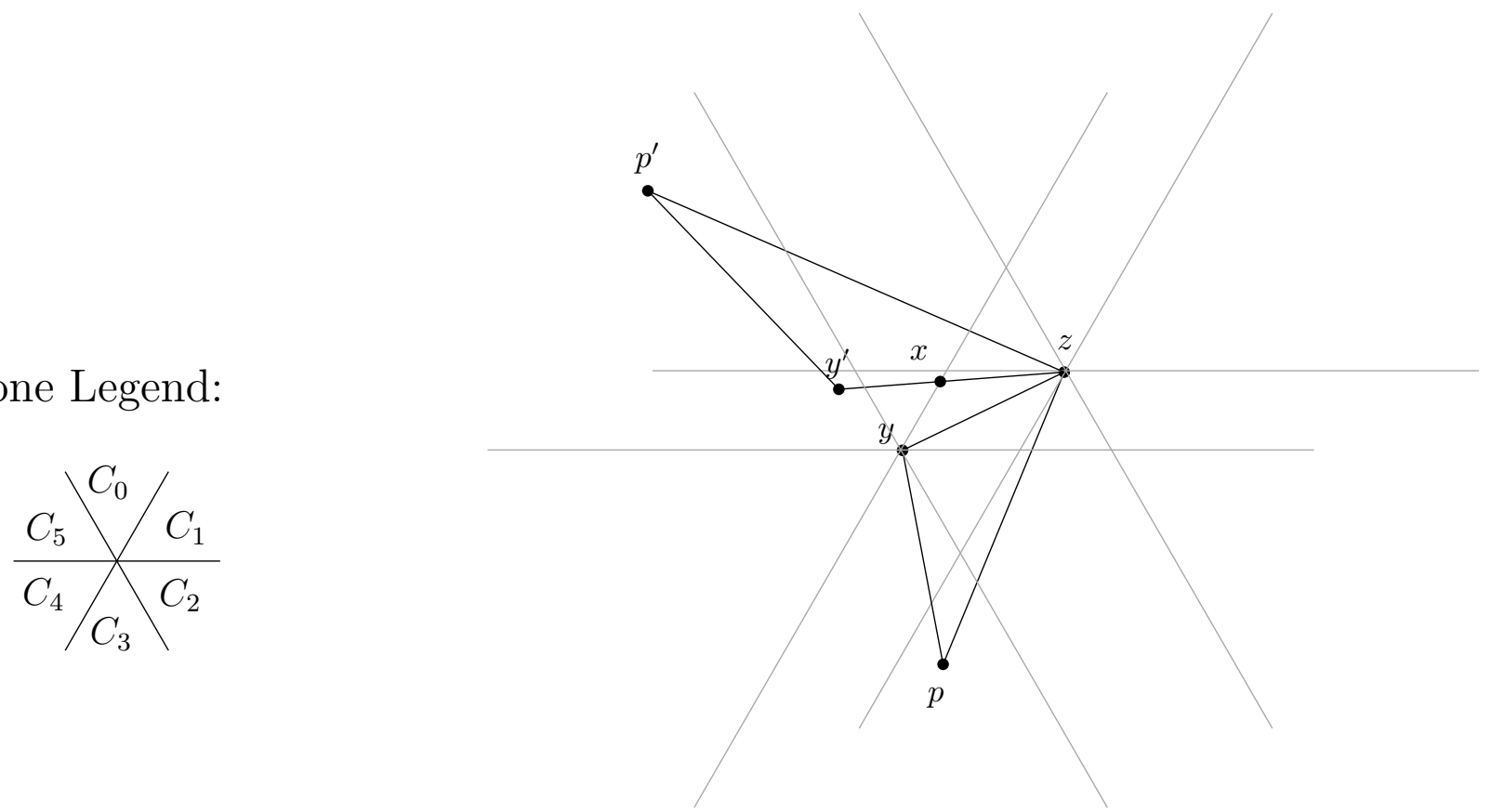

Figure 24: If $(y, z)$ is not in $E_{A}$, there must be a neighbour of $y$ or $z$ in $\triangle(y x z)$.

generality we will assume $[y z]<\left[y^{\prime} z\right]$. We therefore know that $y^{\prime} \notin C_{1}^{y}$. So there must be a vertex $t$ in $C_{1}^{y}$ that is a neighbour of $y$ and closer to $y$ than $z$ counter-clockwise from $(y, z)$. Since $z \in C_{1}^{y}$ and $y^{\prime}$ is not, the counter-clockwise cone boundary of $C_{1}^{y}$ must intersect $\left(y^{\prime}, z\right)$ at a point which we will call $x$. Therefore $t$ must be in triangle $\triangle(x y z)$. See Figure 24 .

Within $\triangle(x y z)$ we take the closest vertex to $z$ and call it $u$. $(u, z)$ must be an edge in $D T(P)$, and $C_{1}^{u}$ is bounded on one side by $(y, z)$, and bounded on the other side by $\left(y^{\prime}, z\right)$, and thus $z$ is the closest point to $u$ in $C_{1}^{u}$. Which means that $(u, z)$ would have been added to $E_{A}$ in AddIncident(), which means that $C_{4}^{z}$ has an edge $(u, z) \in E_{A}$. Since there is an edge of $E_{A}$ in $C_{4}^{z}$, neither $(y, z)$ nor $\left(y^{\prime}, z\right)$ would have been added to $E_{C A N}$ in calls to AddCanonical $(\cdot, \cdot)$ Step $4 \mathrm{~b}$, and neither would be charged to $C_{4}^{z}$.

This leads to the following corollary:

Corollary 4.5.3. Assume an edge $(y, z)$ is added to $E_{C A N}$ in AddCanonical $(p, r)$ Step 4b. and charged to a boundary cone $C_{4}^{z}$. Then of all the edges in $D 8(P)$, only $(y, z)$ is charged 
to $C_{4}^{z}$.

\subsubsection{Edges in Shared Triangles}

The shared triangle is the only scenario where the internal cones of two separate cone neighbourhoods are adjacent on the same vertex.

Consider a shared triangle $\triangle\left(p p^{\prime} s\right)$ with base $\left(p, p^{\prime}\right)$, and assume that $p^{\prime}$ is in $C_{0}^{p},(p, r) \in$ $E_{A}$ is in $C_{0}^{p}$, and $\left(p^{\prime}, r^{\prime}\right) \in E_{A}$ is in $C_{3}^{p^{\prime}}$. Vertex $s$ has adjacent cones inside $\operatorname{Can}_{0}^{(p, r)}$ and $\mathrm{Can}_{3}^{\left(p^{\prime}, r^{\prime}\right)}$. We prove a limit on the number of canonical edges of $p$ and $p^{\prime}$ that were added to $E_{C A N}$ and charged to cones of $s$ inside $\operatorname{Can}_{0}^{(p, r)}$ and $\operatorname{Can}_{3}^{\left(p^{\prime}, r^{\prime}\right)}$.

Lemma 4.5.4. If $\left(s, p^{\prime}\right)$ was added to $E_{C A N}$ and charged to the empty cone of $s$ inside $C a n_{0}^{(p, r)}$, then $(s, p)$ will not be charged to the empty cone of s inside $\operatorname{Can}_{3}^{\left(p^{\prime}, r^{\prime}\right)}$.

Proof. Assume that $\left(p^{\prime}, s\right)$ was added to $E_{C A N}$ by a call to $\operatorname{Add} \operatorname{Canonical}(p, r)$. That implies that $\left(p^{\prime}, s\right)$ is not the first or last edge of $\operatorname{Can}_{i}^{(p, r)}$. Thus we know by Lemma 4.3.16 that $(p, s)$ must be the last edge in $\operatorname{Can}_{3}^{\left(p^{\prime}, r^{\prime}\right)}$, which implies that it is not added by AddCanonical $\left(p^{\prime}, r^{\prime}\right)$.

Otherwise assume that $\left(p, p^{\prime}\right)$ is a canonical edge of $q$, and $(p, s)$ was added to $E_{C A N}$ on a call to $\operatorname{AddCanonical}(q, \cdot)$ in Step $4 \mathrm{c}$. This implies that $\left(p, p^{\prime}\right)$ is in $C_{5}^{q}$. See Figure 25. There are two possible ways to add $\left(p^{\prime}, s\right)$ so that it is charged to the cone of $s$ inside $\operatorname{Can}_{3}^{\left(p^{\prime}, r^{\prime}\right)}$. We show that neither occurs:

1. AddCanonical $(q, \cdot)$ adds $\left(p^{\prime}, s\right)$ to $E_{C A N}$ in Step $4 c$ This implies that $\left(p, p^{\prime}\right)$ is in $C_{4}^{q}$. See Figure 26. However, $(p, s)$ was added in Step 4c, which means that $\left(p, p^{\prime}\right)$ is in $C_{5}^{q}$, which is a contradiction. Thus both edges cannot be added by calls to $\operatorname{AddCanonical}(q, \cdot)$, Step 4c.

2. AddCanonical $(p, r)$ adds $\left(p^{\prime}, s\right)$ to $E_{C A N}$. The shared neighbour $q$ of $p$ and $p^{\prime}$ is not in $C_{0}^{p}$, and thus $\left(p^{\prime}, s\right)$ is the last canonical edge in $\operatorname{Can}_{0}^{(p, r)}$. Thus $\left(p^{\prime}, s\right)$ is not added to $E_{C A N}$ by a call to $\operatorname{AddCanonical}(p, r)$ (by omission, Step 4). 
Cone Numbering:
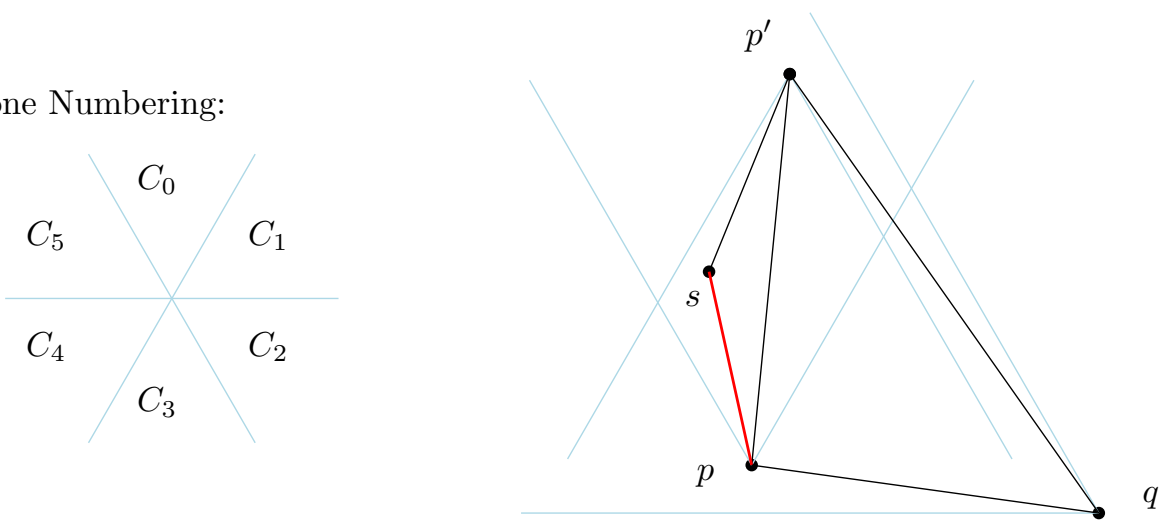

Figure 25: Only $(p, s)$ can be added to $E_{C A N}$ in Step 4c with apex $q$.
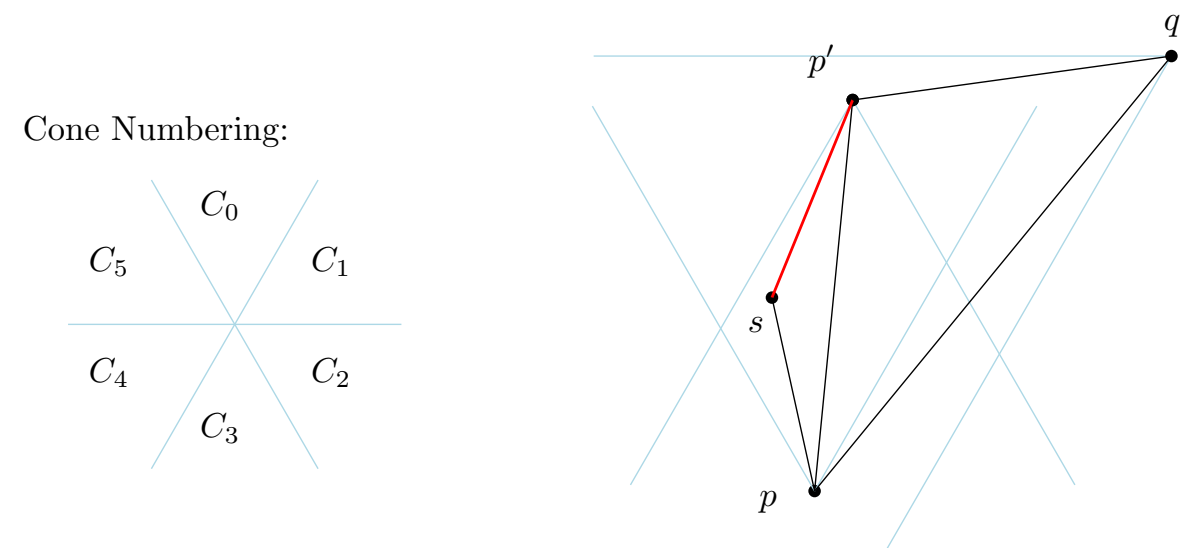

Figure 26: Only $\left(p^{\prime}, s\right)$ can be added to $E_{C A N}$ in Step $4 c$ with apex $q$.

Corollary 4.5.5. If the empty cone of $s$ inside $\operatorname{Can}_{0}^{(p, r)}$ is charged twice by edges of Can ${ }_{0}^{(p, r)}$, then the empty cone of $s$ inside $\operatorname{Can}_{3}^{\left(p^{\prime}, r^{\prime}\right)}$ is charged at most once by edges of $\operatorname{Can}_{3}^{\left(p^{\prime}, r^{\prime}\right)}$.

\subsubsection{The Degree of D8(P)}

Lemma 4.5.6. The edges of $E_{A}$ and $E_{C A N}$ are never charged to the same cone.

Proof. The edges of $E_{A}$ are charged directly to the cone they occupy on each endpoint. We 
know from the charging argument that the edges of $E_{C A N}$ are charged to empty cones, or to a cone that does not contain an edge of $E_{A}$. Thus the edges of $E_{C A N}$ and $E_{A}$ are never charged to the same cone.

Lemma 4.5.7. Consider a cone $C_{i}^{s}$ of a vertex $s$ in $D 8(P)$ that is charged twice by edges of $E_{C A N}$. Then the two neighbouring cones $C_{i-1}^{s}$ and $C_{i+1}^{s}$ are charged at most once by edges of $D 8(P)$.

Proof. Consider an edge $(p, r)$ of $E_{A}$ in $C_{i}^{p}$. A vertex $s$ in $\operatorname{Can}_{i}^{(p, r)}$ can have a cone that is charged twice by edges of $E_{C A N}$ only if it is not the first or last vertex in $\operatorname{Can}_{i}^{(p, r)}$. Additionally, $r$ is not charged twice in any internal cone of $\operatorname{Can}_{i}^{(p, r)}$. Thus assume $s$ is not the first or last vertex in $\operatorname{Can}_{i}^{(p, r)}$, and $s \neq r$.

Each cone $C_{i-1}^{s}$ and $C_{i+1}^{s}$ is either an empty internal cone of $\operatorname{Can}_{i}^{(p, r)}$, or a boundary cone containing a canonical edge of $\operatorname{Can}_{i}^{(p, r)}$ with endpoint $s$. We will consider $C_{i+1}^{s}$ since the cases are symmetric.

If $C_{i+1}^{s}$ is an empty internal cone of $\operatorname{Can}_{i}^{(p, r)}$, then it is only charged for an edge if $s$ is on a shared triangle $\triangle\left(p p^{\prime} s\right)$ and $s$ is not on the base. In this case $C_{i+1}^{s}$ is charged for at most one edge of $E_{C A N}$ by Lemma 4.5.5.

Otherwise $C_{i+1}^{s}$ contains a canonical edge in $\operatorname{Can}_{i}^{(p, r)}$. By our charging scheme we know only empty cones are double charged, and by Lemma 4.5.6 no cone is charged for both an edge of $E_{A}$ and an edge of $E_{C A N}$. Thus $C_{i+1}^{s}$ is either charged for an edge of $E_{A}$, an edge of $E_{C A N}$, or it is not charged.

Theorem 4.5.8. The maximum degree of $D 8(P)$ is at most 8.

Proof. Each edge $(p, r)$ of $E_{A}$ is charged once to the cone of $p$ containing $r$ and once to the cone of $r$ containing $p$. By Lemma 4.2.1, no cone is charged more than once by edges of $E_{A}$.

No edge of $E_{C A N}$ is charged to a cone that is charged by an edge of $E_{A}$ by Lemma 4.5.6. By Lemma 4.5.7, if a cone of a vertex $s$ of $D 8(P)$ is charged twice, then its neighbouring 


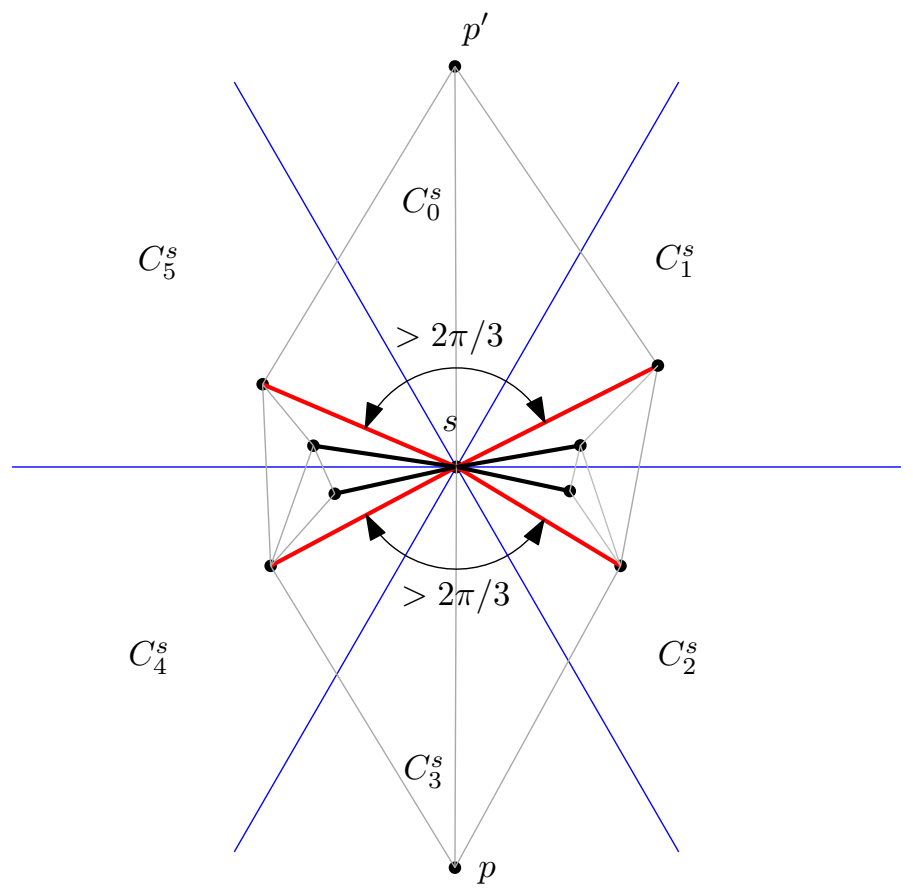

Figure 27: A degree 8 vertex in $D 8(P)$. The red edges belong to $E_{C A N}$, while the black edges belong to $E_{A}$. The light edges are edges of $D T(P)$ that may or may not be in $D 8(P)$.

cones are charged at most once. This implies that there are at most 3 double charged cones on any vertex $s$ in $D 8(P)$. h Assume that we have a vertex $s$ with 3 cones that have been charged twice. A cone of $s$ that is charged twice is an internal cone of some cone neighbourhood $N_{i}^{p}$ by our charging argument. Thus $s$ is endpoint to two canonical edges $(q, s)$ and $(s, t)$ in $N_{i}^{p}$. Note that $\angle(q s t)>2 \pi / 3$ by Lemma 4.3 .8 , and this angle contains the cone of $s$ that is charged twice. Thus to have 3 cones charged twice, the total angle around $s$ would need to be $>2 \pi$, which is impossible. Thus there are at most two double charged cones on $s$, which gives us a maximum degree of 8 .

See Figure 27 for an example of a point set $P$ for which the maximum degree of $D 8(P)$ is equal to 8 . 


\section{Chapter 5}

\section{$\mathrm{D} 8(\mathrm{P})$ is a Spanner}

\subsection{Introduction and Preliminaries}

We will prove that $D 8(P)$ is a spanner of $D T(P)$ with a spanning ratio of $\left(1+\frac{\theta}{\sin \theta}\right)=$

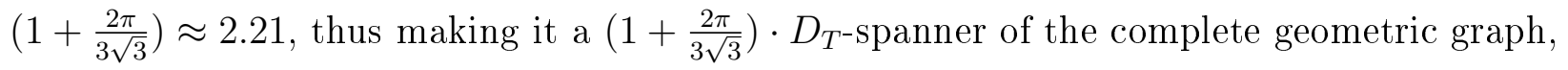
where $D_{T}$ is the spanning ratio of the Delaunay triangulation. As of this writing, the current best bound of the spanning ratio of the Delaunay triangulation is 1.998 [7], which makes $D 8(P)$ approximately a 4.42-spanner of the complete graph.

We begin by defining the ideal path, and proving the spanning ratio of an ideal path with respect to the graph $D T(P)$.

Before we define the ideal path, we first prove the following:

Lemma 5.1.1. Let $(p, r)$ be an edge in $E_{A}$ in the cone $C_{i}^{p}$. Then Can $_{i}^{(p, r)}$ forms a path.

Proof. We prove by contradiction. Note that $\operatorname{Can}_{i}^{(p, r)}$ is a collection of paths. Assume that there are at least two paths in this collection. Without loss of generality, let $i=0$. Let $(a, b)$ and $(y, z)$ be the first and last edge respectively in $\operatorname{Can}_{0}^{(p, r)}$. Thus of all the vertices in $N_{0}^{p} \backslash\{p\}$ between $a$ and $z$ there exists at least one consecutive subset $T$ where for each $t_{j} \in T, 0 \leq j<|T|,\left[p t_{j}\right]<[p r]$. We consider the vertex $t_{k} \in T,\left[p t_{k}\right] \leq\left[p t_{j}\right]$, for all $t_{j} \in T, 0 \leq j<|T|$. Since $\left[p t_{k}\right]<[p r]$, AddIncident $(L)$ examined $\left(p, t_{k}\right)$ before $(p, r)$. Thus 


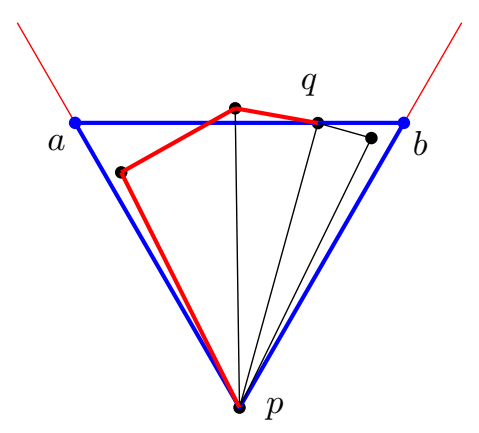

Figure 28: The canonical triangle $T_{p q}$ in blue. Note, a possible canonical path (in red) does not need to be inside the canonical triangle.

when $\left(p, t_{k}\right)$ was examined, $C_{i}^{p}$ contained no edges of $E_{A}$ with endpoint $p$. Since $\left(p, t_{k}\right)$ was not added to $E_{A}$, there must have been an edge of $E_{A}$ with endpoint $t_{k}$ in $C_{3}^{t_{k}}$. However, we know $\left[p t_{k-1}\right] \leq\left[p t_{k}\right]$ and $\left[p t_{k+1}\right] \leq\left[p t_{k}\right]$ (whether or not $t_{k-1}$ and $t_{k+1}$ are in $T$ ). Thus neither $\left(t_{k}, t_{k-1}\right)$ nor $\left(t_{k}, t_{k+1}\right)$ can be in $C_{3}^{t_{k}}$. Since $\triangle\left(p t_{k} t_{k-1}\right)$ and $\triangle\left(p t_{k} t_{k+1}\right)$ are triangles in $D T(P)$, the only edge with endpoint $t_{k}$ in $C_{3}^{t_{k}}$ is $\left(p, t_{k}\right)$. This means that $\left(p, t_{k}\right)$ would have been added to $E_{A}$ instead of $(p, r)$, which is a contradiction.

Definition 5.1.2. Consider an edge $(p, r)$ in $C_{i}^{p}$ in $E_{A}$, and the graph $\operatorname{Can}_{i}^{(p, r)}$. An ideal path is a simple path from $p$ to any vertex in $\operatorname{Can}_{i}^{(p, r)}$ using the edges of $(p, r) \cup \operatorname{Can}_{i}^{(p, r)}$.

We also introduce the concept of the canonical triangle, as seen in the paper by Bose et al. [14]:

Definition 5.1.3. Consider a vertex $q$ in the cone $C_{i}^{p}$ of vertex $p$. Take the line through $q$ perpendicular to the bisector of $C_{i}^{p}$. Let $a$ and $b$ be the intersection points of this line with the cone $C_{i}^{p}$. Then the canonical triangle $T_{p q}$ is the triangle $\triangle(p a b)$. See Figure 28

Consider an edge $(p, r)$ in $C_{i}^{p}$ in $E_{A}$, and the graph $\operatorname{Can}_{i}^{(p, r)}$. We will prove that the length of the ideal path from $p$ to $q$ is not greater than $|p a|+\frac{\theta}{\sin \theta}|a q|$, where $a$ is the corner of the canonical triangle to the side of $(p, q)$ that has $r$, and $\theta=\pi / 3$ is the cone angle.

We then use ideal paths to prove there exists a path with bounded spanning ratio between 
any two vertices $p$ and $q$ in $D 8(P)$, where $(p, q)$ is an edge in $D T(P)$. We prove a bound on the length of the path from $p$ to $q$ of $|p a|+\frac{\theta}{\sin \theta}|a q|$.

We note that the distance $|p a|+\frac{\theta}{\sin \theta}|a q|$ is with respect to the canonical triangle $T_{p q}$ rather than the Euclidean distance $|p q|$. To finish the proof we show that:

$$
|p a|+\frac{\theta}{\sin \theta}|a q| \leq\left(1+\frac{\theta}{\sin \theta}\right)|p q|
$$

\subsection{Spanning Ratio of Ideal Paths}

We begin with a couple of well-known geometric lemmas. The first is an observation regarding the relative lengths of convex paths, when one resides inside the other.

Lemma 5.2.1. If a convex body $C$ is contained within another convex body $C^{\prime}$, then the perimeter of $C^{\prime}$ is longer than $C .[15]$, page 42.

The next lemma is a well known result traditionally called "The Inscribed Angle Theorem".

Lemma 5.2.2. Consider 3 points $p, q, s$ on the boundary of a circle $O$ with center $o$, such that $\angle(p q s)=\alpha$. Let $A$ be the arc of $O$ from $p$ to $s$ that does not go through $q$, and let $\bar{A}$ be the arc of $O$ from $p$ to $s$ through $q$. Then the angle $\angle(p o s)$ facing $A$ is equal to $2 \alpha$. Further, the angles $\angle(p q s)$ facing $A$ is the same for any point $p$ that is on $\bar{A}$.

That allows us to establish this result:

Lemma 5.2.3. Let $O$ be a circle through points $p$ and $q$ and $r$ in clockwise order, and let $\alpha$ denote the angle $\angle(q p r)$. Then the length of the arc from $q$ to $r$ on the boundary of $D_{p, q, r}$ is

$$
\frac{\alpha}{\sin \alpha}|q r|
$$




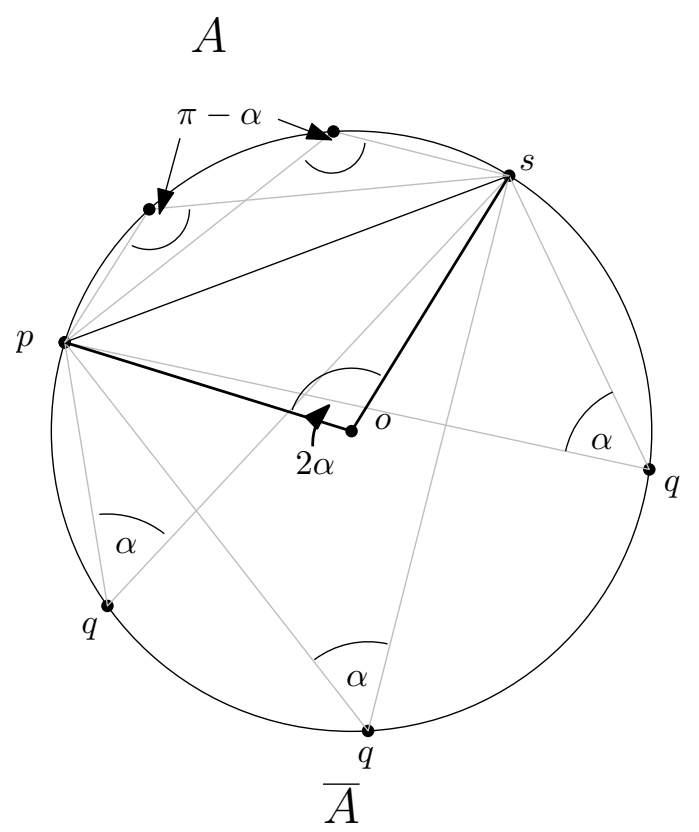

Figure 29: The Inscribed Angle Theorem

Proof. From the center point of $O$, the angle between $q$ and $s$ is $2 \alpha$ by Lemma 5.2.2. Thus the arc length between $q$ and $r$ is $2 \alpha R$, where $R$ is the radius of $O$. Also, $|q r|=2 \sin \alpha R$, which means $R=\frac{|q r|}{2 \sin \alpha}$. Thus the arc length between $q$ and $r$ is equal to:

$$
\begin{aligned}
2 \alpha R & =\frac{2 \alpha}{2 \sin \alpha}|q r| \\
& =\frac{\alpha}{\sin \alpha}|q r|
\end{aligned}
$$

which completes the proof. See Figure 30.

Lemma 5.2.4. Consider the restricted neighbourhood $N_{p}^{(r, q)}$ in $D T(P)$ in the cone $C_{i}^{p}$. Let $O_{p, r, q}$ be the circle through the points $p, q$, and $r$. Then there are no points of $P$ in $O_{p, r, q}$ to the side of $(p, r)$ that does not contain $q$. Likewise there are no points of $P$ in $O_{p, r, q}$ to the side of $(p, q)$ that does not contain $r$. 


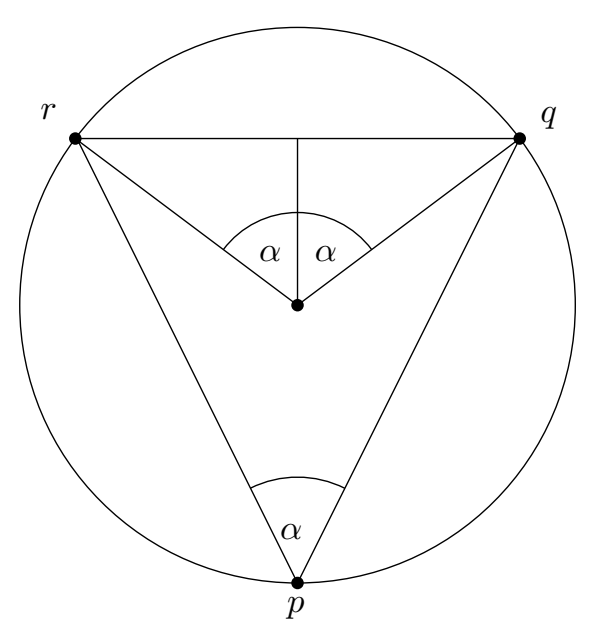

Figure 30: Length of arc between $r$ and $q$.

Proof. Since the cases are symmetric, we prove that there are no points of $P$ in the region $R$ of $O_{p, r, q}$ to the side of $(p, r)$ that does not contain $q$. We prove by contradiction. Thus assume there is a point $t$ in $R$. Then the circle $O_{p, t, r}$ contains $q$ and the circle $O_{p, r, q}$ contains $t$, thus there is no circle through $p$ and $r$ that is empty of points of $P$. Thus $(p, r)$ cannot be a Delaunay edge, which is a contradiction to our definition of restricted neighbourhood. See Figure 31 .

Lemma 5.2.5. Consider the restricted neighbourhood $N_{p}^{(r, q)}$ in cone $C_{i}^{p}$. Let $r q$ be the directed line from $r$ to $q$, and assume there are no neighbours of $p$ in $N_{p}^{(r, q)}$ right of rq. If $(r, q)$ is not an edge in $N_{p}^{(r, q)}$, then there is a vertex $a \in N_{p}^{(r, q)}$ such that the circle $O_{r, a, q}$ is empty of vertices of $P$ left of $r q$.

Proof. We prove by contradiction, thus assume that we have found a vertex $a$ left of $r q$ such that $O_{r, a, q}$ is empty of vertices of $P$ left of $r q$, and $a$ is not in $N_{p}^{(r, q)}$. Note vertex a must exist, otherwise $(r, q)$ is on the convex hull and thus in $N_{p}^{(r, q)}$. Since the region of $N_{p}^{(r, q)}$ is empty of vertices of $P, a$ must be outside of $N_{p}^{(r, q)}$.

We look at two cases: 


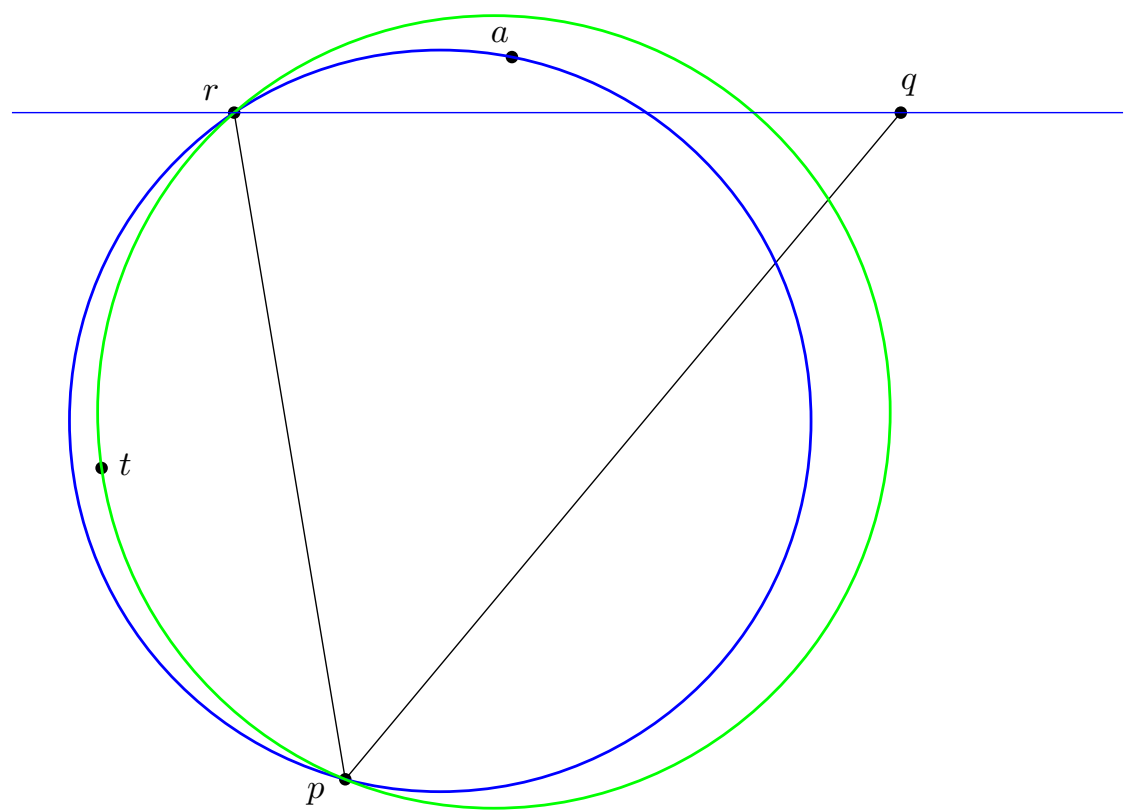

Figure 31: If there is a point t, then $(p, r)$ is not an edge in $D T(P)$.

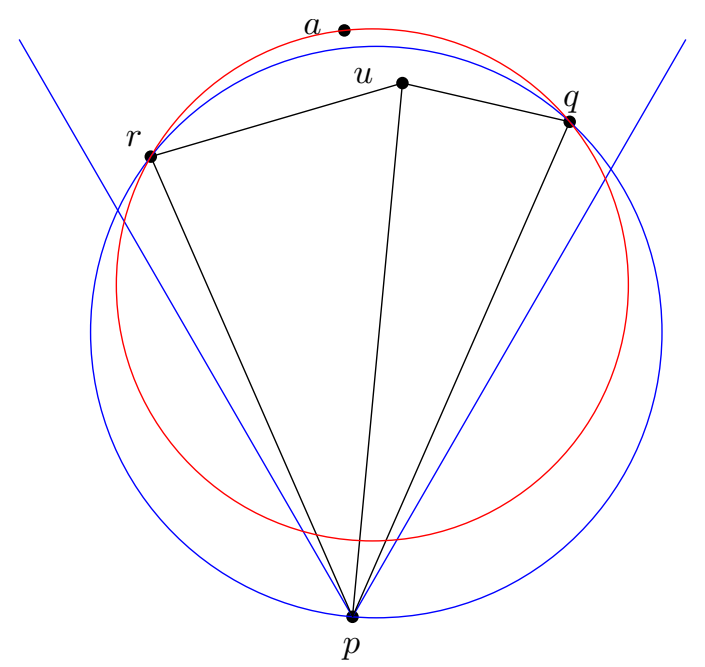

Figure 32: Circle $O_{r, a, q}$ contains $u$. 
1. $a$ is outside of $O_{p, r, q}$ : Since $(r, q)$ is not in $D T(P)$, there is at least one vertex $u$ in $N_{p}^{(r, q)} \backslash\{p, r, q\}$. By Lemma 4.3.7 and our initial assumption that $N_{p}^{(r, q)}$ contains no neighbours of $p$ to the right of $r q, u$ must be in $O_{p, r, q}$ to the left of $r q$. Since $a$ is outside of $O_{p, r, q}$, the arc of $O_{r, a, q}$ to the left of $r q$ contains the arc of $O_{p, r, q}$ to the left of $r q$. Thus $u$ is in $O_{r, a, q}$ to the left of $r q$, which is a contradiction to our selection of vertex $a$. See Figure 32 .

2. $a$ is inside $O_{p, r, q}$ : Since $\angle(r p q)<\pi / 3$ (since it is in a cone), and $a$ is inside $O_{p, r, q}, a$ must be positioned radially between two consecutive edges with endpoint $p$ in $N_{p}^{(r, q)}$. Call these edges $(p, u)$, and $(p, v)$. Note that $\triangle(p u v)$ is a triangle in $D T(P)$, and thus the circle $O_{p, u, v}$ does not contain $a$ by the empty circle property of the Delaunay triangulation. This implies that, since $p, u, a$, and $v$ form a convex quadrilateral with $p$ and $a$ across the diagonal, any circle through $p$ and $a$ must contain at least one of $u$ or $v$.

Since $a$ is inside $O_{p, r, q}, O_{r, a, q}$ contains $p$. Thus we can draw the circle $O_{1}$ through $a$ and $p$ tangent to $O_{r, a, q}$. The portion of $O_{1}$ to the left of $r q$ is contained in $O_{r, a, q}$, and thus does not contain any points of $P$. But any circle through $a$ and $p$ must contain at least one of $u$ and $v$, and $u$ and $v$ are to the left of $r q$, which is a contradiction. See Figure 33

Thus, if $(r, q)$ is not an edge in $N_{p}^{(r, q)}$, there is a neighbour $a$ of $p$ in $N_{p}^{(r, q)}$ such that $O_{r, a, q}$ is empty of vertices of $P$ left of $r q$. See Figure 33 .

We now turn to a lemma from the paper of Bose and Keil [16] that tells us the length of a path between two points in the Delaunay triangulation of a set of vertices. We provide a slightly modified and truncated version that suits our needs. The lemma of Bose and Keil does not provide an explicit construction. We apply the lemma to a restricted neighbourhood, and are able to provide a construction of the path along with an upper bound on its length. 


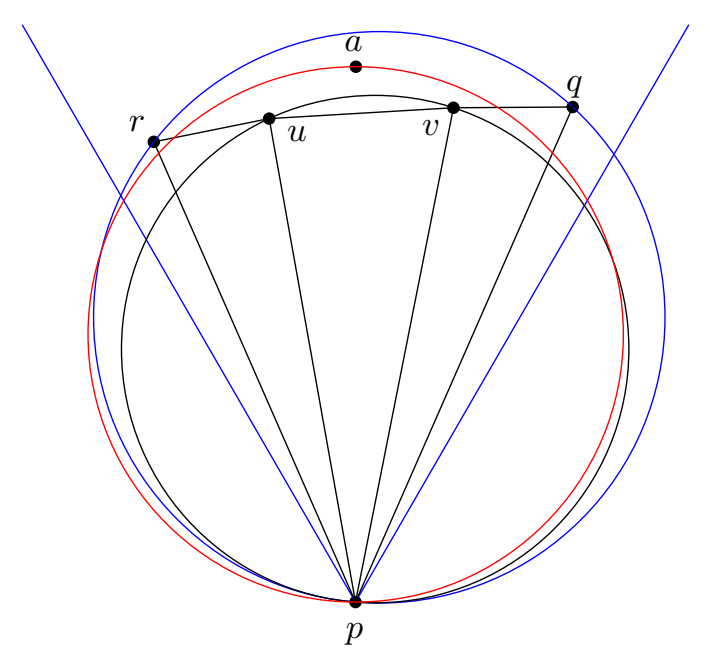

Figure 33: A circle through a and $p$ contains $u$ or $v$.

Lemma 5.2.6. Consider the restricted neighbourhood $N_{p}^{(r, q)}$ in $D T(P)$ in the cone $C_{i}^{p}$. Let $\alpha=\angle(r p q)<\pi / 3$. If no point of $P$ lies in the triangle $\triangle(p r q)$ then there is a path from $r$ to $q$ in $D T(P)$, using canonical edges of $p$, whose length satisfies:

$$
\delta(r, q) \leq|r q| \frac{\alpha}{\sin \alpha}
$$

Proof. Let $o$ be the center of $O_{p, r, q}$, and let $\beta=\angle($ roq $)=2 \alpha$.

Lemma 5.2 .4 and the assumption that no vertices of $P$ lie in the triangle $\triangle(p r q)$ imply that there are no vertices of $P$ in $O_{p, r, q}$ to the right of directed line segment $r q$.

We proceed by induction on the number of vertices in $N_{p}^{(r, q)}$. If there are only 3 vertices in $N_{p}^{(r, q)}$, then $(r, q)$ is an edge in $D T(P)$, and the path from $r$ to $q$ has length $|r q|<|r q| \frac{\alpha}{\sin \alpha}$ and we are done.

Now assume that the inductive hypothesis holds for all restricted neighbourhoods with fewer vertices than $N_{p}^{(r, q)}$. Assume $N_{p}^{(r, q)}$ has more than 3 vertices, otherwise we are done by the same argument as above.

Lemma 5.2.4 tells us that there is a vertex $a$ in $N_{p}^{(r, q)}$ where $O_{r, a, q}$ is empty of vertices of $P$ left of $r q$.

Let $O_{1}$ be the circle through $r$ and $a$ with center $o_{1}$ on the line segment $(o, r)$. Let $O_{2}$ be 
the circle through $a$ and $q$ whose center $o_{2}$ lies on the line segment $(o, q)$. Let $\alpha_{1}=\angle\left(r o_{1} a\right)$ and let $\alpha_{2}=\angle\left(a o_{2} q\right) . N_{p}^{(r, a)}$ and $N_{p}^{(a, q)}$ have fewer vertices than $N_{p}^{(r, q)}$, and $O_{1}$ is empty of vertices of $P$ to the right of directed segment $r a$, and $O_{2}$ is empty of vertices of $P$ to the right of directed line segment aq. Thus by the inductive hypothesis:

$$
\begin{aligned}
\delta(r, q) & =\delta(r, a)+\delta(a, q) \\
& =|r a| \frac{\alpha_{1}}{\sin \alpha_{1}}+|a q| \frac{\alpha_{2}}{\sin \alpha_{2}}
\end{aligned}
$$

Let $r^{\prime} \neq r$ be the intersection of $O_{1}$ and $r q$, and let $q^{\prime} \neq q$ be the intersection of $O_{2}$ and $r q$. Since $\beta<\pi, O_{1}$ and $O_{2}$ overlap. Let $O_{3}$ be the circle through $q^{\prime}$ and $r^{\prime}$ with center $o_{3}$ on the intersection of the line segment between $o_{1}$ and $r^{\prime}$ and the line segment between $o_{2}$ and $q^{\prime}$. See Figure 34 .

Triangles $\triangle(r o q), \triangle\left(r O_{1} r^{\prime}\right), \triangle\left(q^{\prime} O_{2} q\right)$, and $\triangle\left(q^{\prime} o_{3} r^{\prime}\right)$ are all similar isosceles triangles. Thus by Lemmas 5.2.2 and 5.2.3 the length of the arc of $O_{1}$ left of $r q$ is $\left|r r^{\prime}\right| \frac{\alpha}{\sin \alpha}$, the length of the arc of $O_{2}$ left of $r q$ is $\left|q^{\prime} q\right| \frac{\alpha}{\sin \alpha}$, and the length of the arc of $O_{3}$ left of $r q$ is $\left|q^{\prime} r^{\prime}\right| \frac{\alpha}{\sin \alpha}$.

Note that $O_{3}$ is completely contained in the intersections of $O_{1}$ and $O_{2}$. Let $A_{1}$ be the arc of $O_{1}$ left of $r q$ from $a$ to $r^{\prime}$, and let $A_{2}$ be the arc of $O_{2}$ left of $r q$ from $a$ to $q^{\prime}$. Note that $A_{1} \cap A_{2}$ is a convex shape from $q^{\prime}$ to $r^{\prime}$ that contains the arc of $O_{3}$ left of $r q$. Thus $\left|A_{1} \cap A_{2}\right| \geq\left|q^{\prime} r^{\prime}\right| \frac{\alpha}{\sin \alpha}$ by convexity (Lemma 5.2.1).

We observe that: 


$$
\begin{aligned}
\delta(r, q) & =\delta(r, a)+\delta(a, q) \\
& =|r a| \frac{\alpha_{1}}{\sin \alpha_{1}}+|a q| \frac{\alpha_{2}}{\sin \alpha_{2}} \\
& =\left|r r^{\prime}\right| \frac{\alpha}{\sin \alpha}+\left|q^{\prime} q\right| \frac{\alpha}{\sin \alpha}-\left|A_{1} \cap A_{2}\right| \\
& \leq\left|r r^{\prime}\right| \frac{\alpha}{\sin \alpha}+\left|q^{\prime} q\right| \frac{\alpha}{\sin \alpha}-\left|q^{\prime} r^{\prime}\right| \frac{\alpha}{\sin \alpha} \\
& =|r q| \frac{\alpha}{\sin \alpha}
\end{aligned}
$$

as required.

Now we prove the following:

Lemma 5.2.7. Consider the restricted neighbourhood $N_{p}^{(r, q)}$ and without loss of generality let $N_{p}^{(r, q)}$ be in $C_{0}^{p}$. Let $\alpha=\angle(r p q)$. Let $r_{q} \neq p$ be the point where the line through $p$ and $r$ intersects the canonical triangle $T_{p q}$. Let $q_{r} \neq p$ be the point where the edge $(p, q)$ intersects $T_{p r}$. If [pr] is the shortest edge of all edges in $N_{p}^{(r, q)}$ with endpoint $p$, then the distance from $r$ to $q$ using the canonical edges of $p$ in $N_{p}^{(r, q)}$ is at most:

$$
\max \left\{\left|r r_{q}\right|,\left|q_{r} q\right|\right\}+\left|r_{q} q\right| \frac{\alpha}{\sin \alpha}
$$

Proof. Let $\delta(r, q)$ be the length of the path between $r$ and $q$ in $N_{p}^{(r, q)}$. We will prove by induction on the number of vertices in $N_{p}^{(r, q)}$.

If there are only three vertices in $N_{p}^{(r, q)}$, then $(r, q)$ is an edge in $N_{p}^{(r, q)}$. Since $|r q| \leq$ $\max \left\{\left|r r_{q}\right|,\left|q_{r} q\right|\right\}+\left|r_{q} q\right| \frac{\alpha}{\sin \alpha}$, we are done.

Otherwise assume there are more than 3 vertices in $N_{p}^{(r, q)}$. Consider the edge $(p, a) \in$ $N_{p}^{(r, q)}$, such that $[p a] \leq[p t]$, for all $(p, t) \in N_{p}^{(r, q)} \backslash\{r, q\}$. Let $\alpha_{1}=\angle(r p a)$. We consider two 


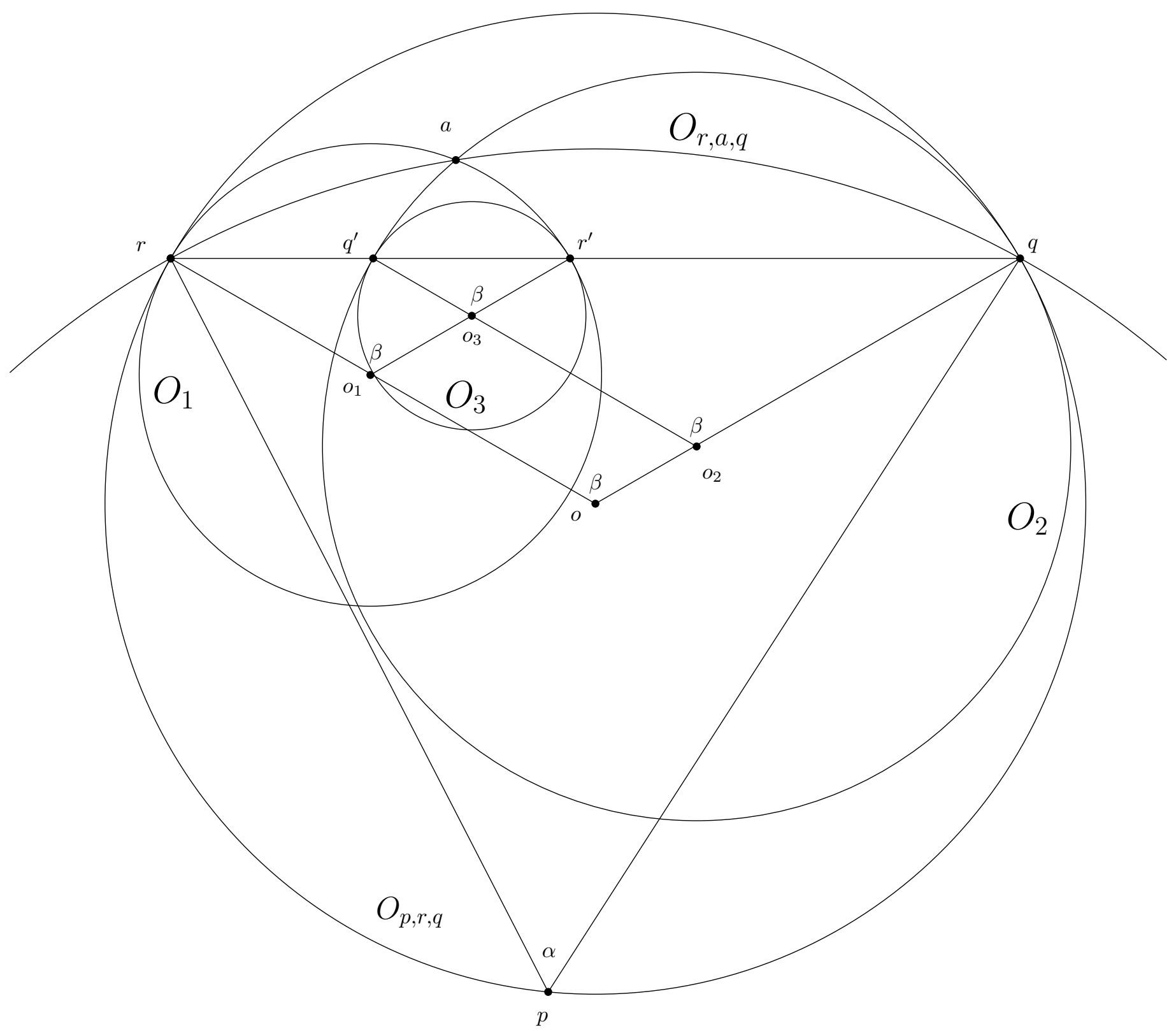

Figure 34: Lemma 5.2.6 
cases:

1. If $[p a]>[p q]$, then $[p r]$ and $[p q]$ are the shortest edges in $N_{p}^{(r, q)}$, which implies that there are no points in $\triangle(p r q)$. Thus from Lemma 5.2.6, the length of the path from $r$ to $q$ is at most $|r q| \frac{\alpha}{\sin \alpha}$. We have

$$
\begin{aligned}
\delta(r, q) & \leq|r q| \frac{\alpha}{\sin \alpha} \\
& \leq\left|r r_{q}\right|+\left|r_{q} q\right| \frac{\alpha}{\sin \alpha}
\end{aligned}
$$

by convexity (Lemma 5.2.1) and thus

$$
\begin{aligned}
\delta(r, q) & \leq\left|r r_{q}\right|+\left|r_{q} q\right| \frac{\alpha}{\sin \alpha} \\
& \leq \max \left\{\left|r r_{q}\right|,\left|q_{r} q\right|\right\}+\left|r_{q} q\right| \frac{\alpha}{\sin \alpha}
\end{aligned}
$$

which satisfies the inductive hypothesis. See Figure 35

2. If $[p a]<[p q]$, let $\alpha_{1}=\angle(r p a)$ and $\alpha_{2}=\angle(a p q)$. Since $[p r] \leq[p a]$ we can apply the inductive hypothesis on $N_{p}^{(r, a)}$. Let $r_{a}$ be the point where the line through $p$ and $r$ intersects the horizontal line through $a$, and let $a_{r}$ be the point where the line through $p$ and $a$ intersects the horizontal line through $r$. See Figure 36. Then by the inductive hypothesis:

$$
\delta(r, a)=\max \left\{\left|r r_{a}\right|,\left|a_{r} a\right|\right\}+\left|r_{a} a\right| \frac{\alpha_{1}}{\sin \alpha_{1}}
$$

Since $[p a] \leq[p q]$ we can apply the inductive hypothesis on $N_{p}^{(a, q)}$. Let $a_{q} \neq p$ be the 


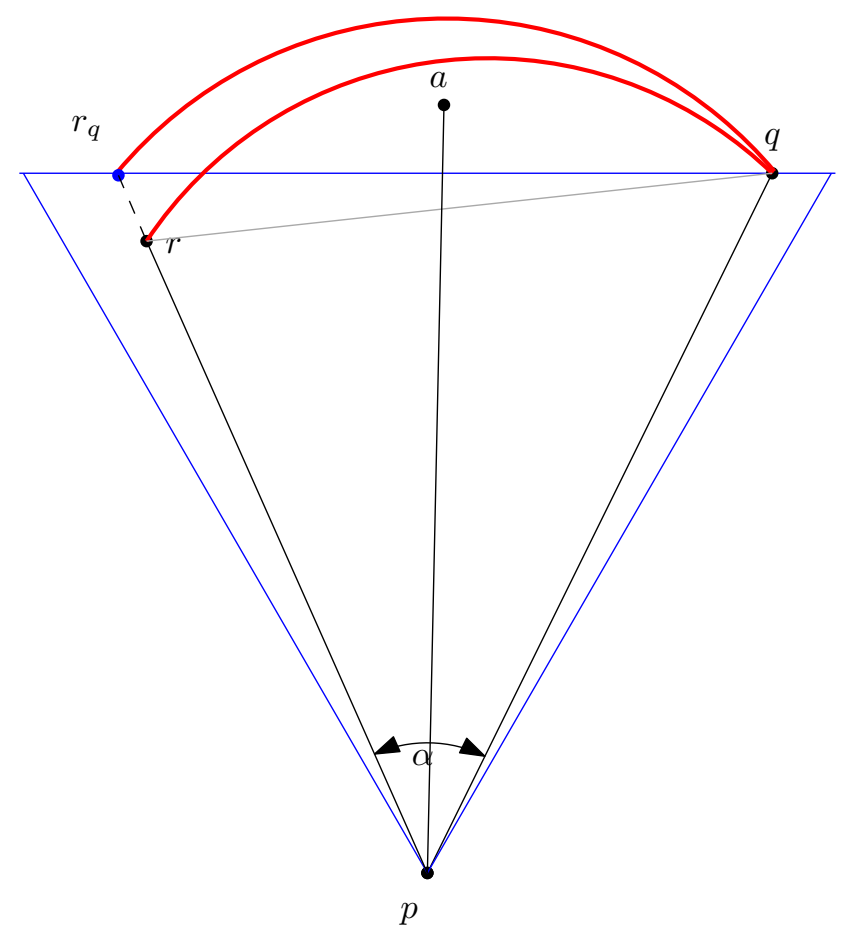

Figure 35: If $|p a| \geq|p q|$, apply Lemma 5.2.6.

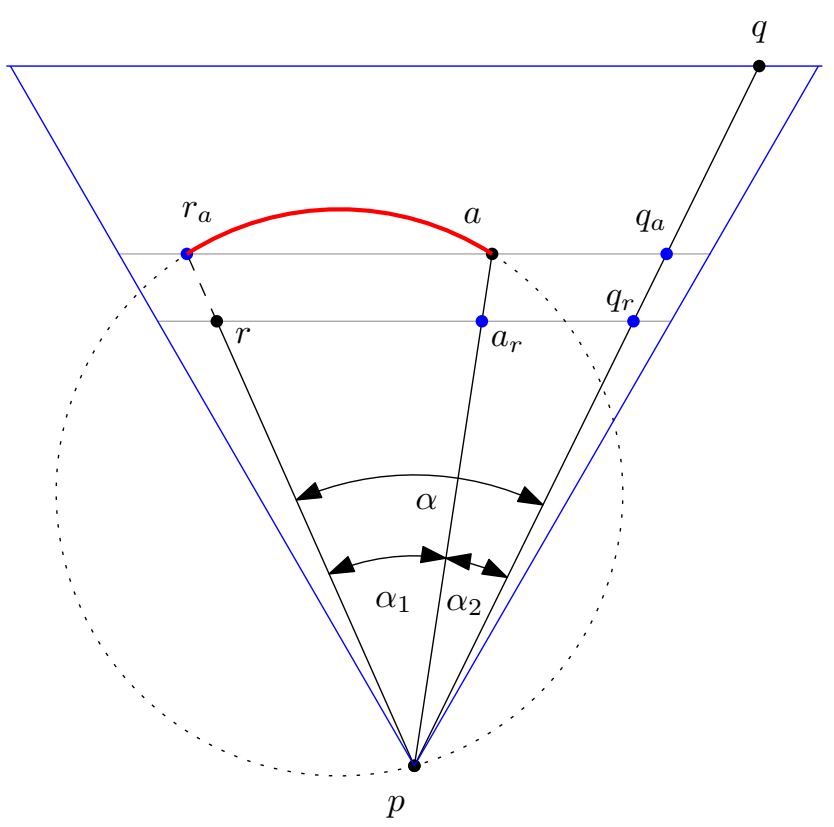

Figure 36: If the vertex $a$ is in $\triangle(p r q)$, we proceed by induction from $r$ to a. 


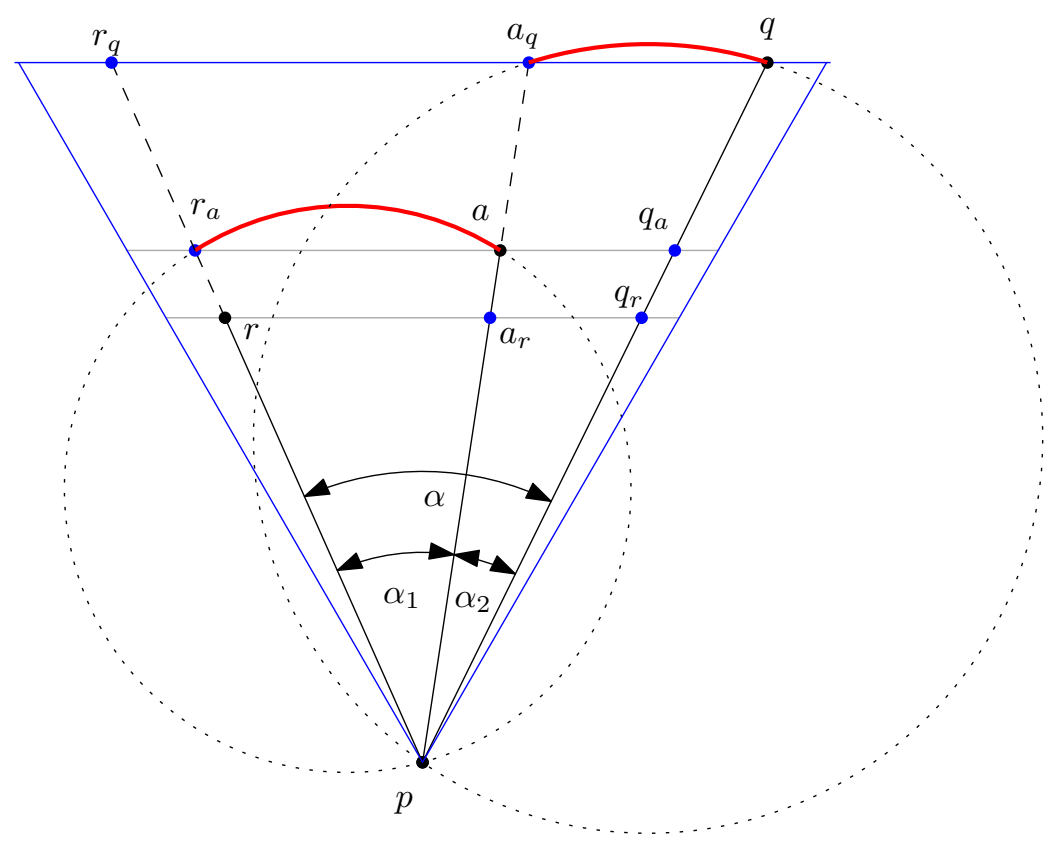

Figure 37: Proceed by induction from a to $q$.

point where the line through $a$ and $p$ exits $T_{p q}$, and let $q_{a} \neq p$ be the point where $(p, q)$ intersects $T_{p a}$, and let $\alpha_{2}=\angle(a p q)$. See Figure 37 . Then by the inductive hypothesis:

$$
\delta(a, q)=\max \left\{\left|a a_{q}\right|,\left|q_{a} q\right|\right\}+\left|a_{q} q\right| \frac{\alpha_{2}}{\sin \alpha_{2}}
$$

We have $\alpha_{1} \leq \alpha$ and $\alpha_{2} \leq \alpha$ and $\frac{\alpha}{\sin \alpha}$ is an increasing function for the interval $0<\alpha<\pi / 3$. Also note that $\left|p a_{q}\right| \leq \max \left\{\left|p r_{q}\right|,|p q|\right\}$. Thus: 


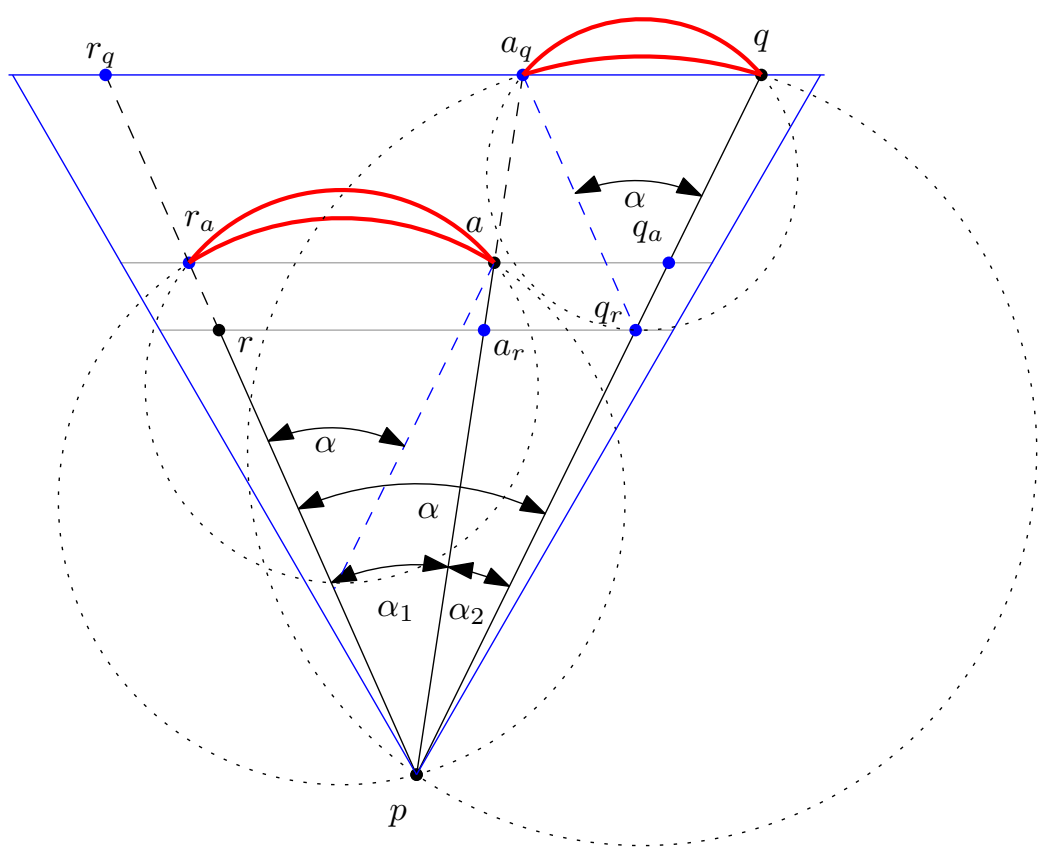

Figure 38: $\left|r_{a} a\right| \frac{\alpha_{1}}{\sin \alpha_{1}}<\left|r_{a} a\right| \frac{\alpha}{\sin \alpha}$ and $\left|a_{q} q\right| \frac{\alpha_{2}}{\sin \alpha_{2}}<\left|a_{q} q\right| \frac{\alpha}{\sin \alpha}$

$$
\begin{aligned}
\delta(r, q) & =\max \left\{\left|r r_{a}\right|,\left|a_{r} a\right|\right\}+\max \left\{\left|a a_{q}\right|,\left|q_{a} q\right|\right\}+\left|r_{a} a\right| \frac{\alpha_{1}}{\sin \alpha_{1}}+\left|a_{q} q\right| \frac{\alpha_{2}}{\sin \alpha_{2}} \\
& \leq \max \left\{\left|r r_{q}\right|,\left|q_{r} q\right|\right\}+\left|r_{a} a\right| \frac{\alpha_{1}}{\sin \alpha_{1}}+\left|a_{q} q\right| \frac{\alpha_{2}}{\sin \alpha_{2}} \\
& \leq \max \left\{\left|r r_{q}\right|,\left|q_{r} q\right|\right\}+\left|r_{q} a_{q}\right| \frac{\alpha_{1}}{\sin \alpha_{1}}+\left|a_{q} q\right| \frac{\alpha_{2}}{\sin \alpha_{2}} \\
& \leq \max \left\{\left|r r_{q}\right|,\left|q_{r} q\right|\right\}+\left|r_{q} a_{q}\right| \frac{\alpha}{\sin \alpha}+\left|a_{q} q\right| \frac{\alpha}{\sin \alpha} \\
& \leq \max \left\{\left|r r_{q}\right|,\left|q_{r} q\right|\right\}+\left|r_{q} q\right| \frac{\alpha}{\sin \alpha}
\end{aligned}
$$

as required.

Figures 38, 39, 40.

We can now prove the main lemma of this section:

Lemma 5.2.8. Consider the edge $(p, r)$ in $E_{A}$, located in $C a n_{i}^{p}$, and the associated 


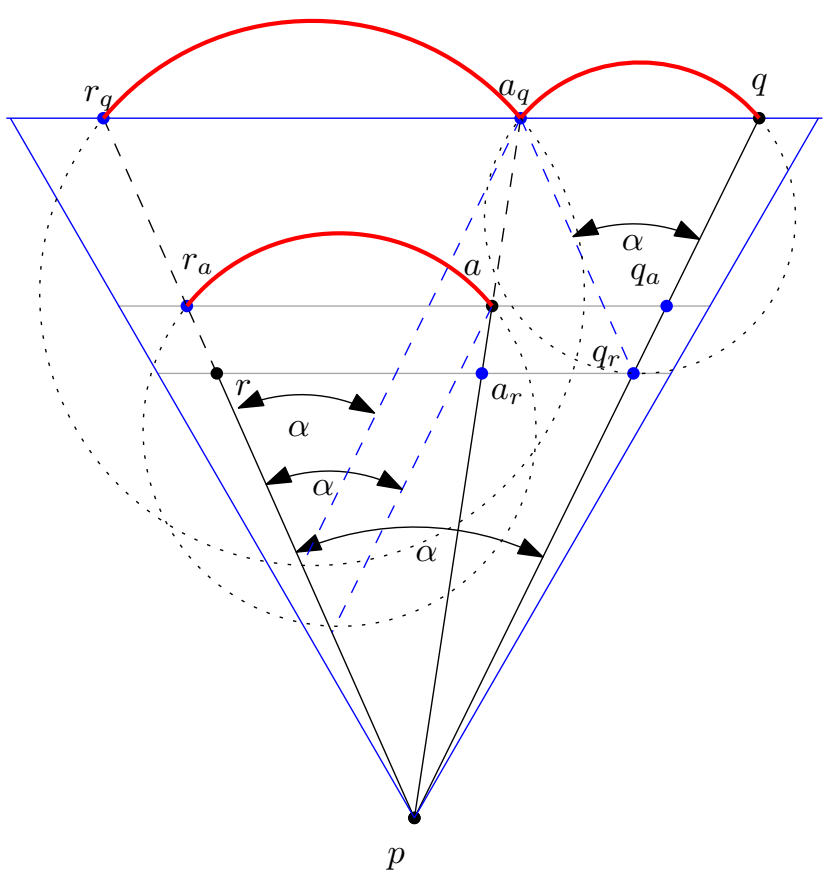

Figure 39: The distance $\left|r_{a} a\right|<\left|r_{q} a_{q}\right|$

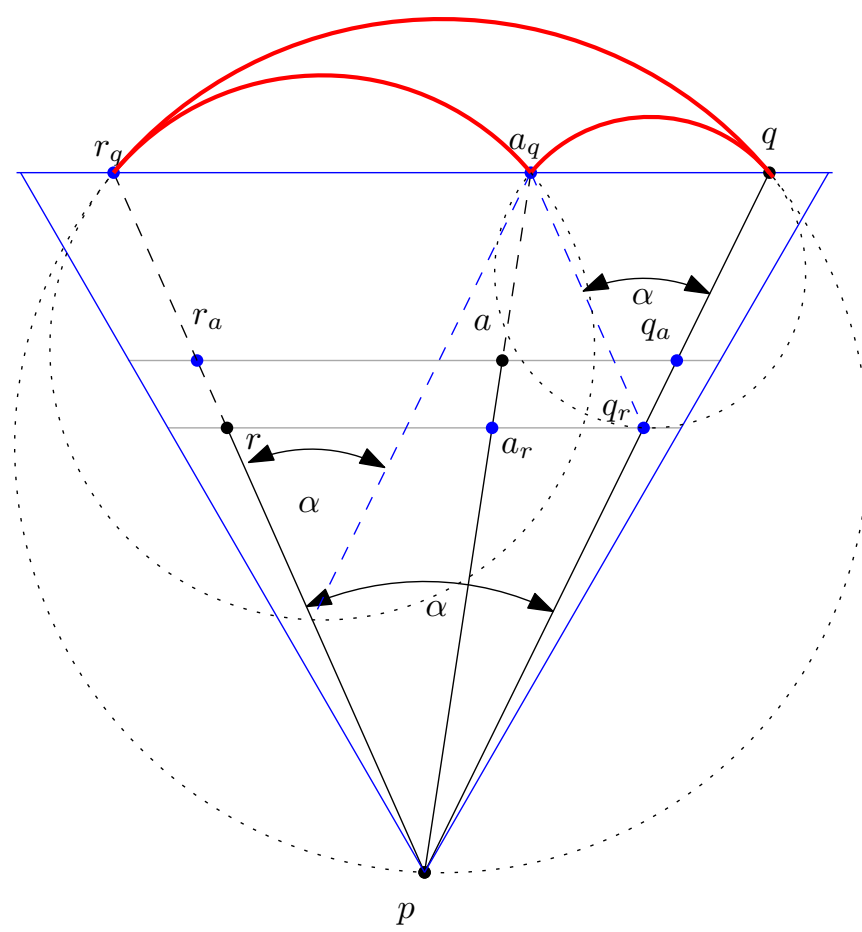

Figure 40: The distances across the arcs are $\left|r_{q} a_{q}\right| \frac{\alpha}{\sin \alpha}+\left|a_{q} q\right| \frac{\alpha}{\sin \alpha}=\left|r_{q} q\right| \frac{\alpha}{\sin \alpha}$ 


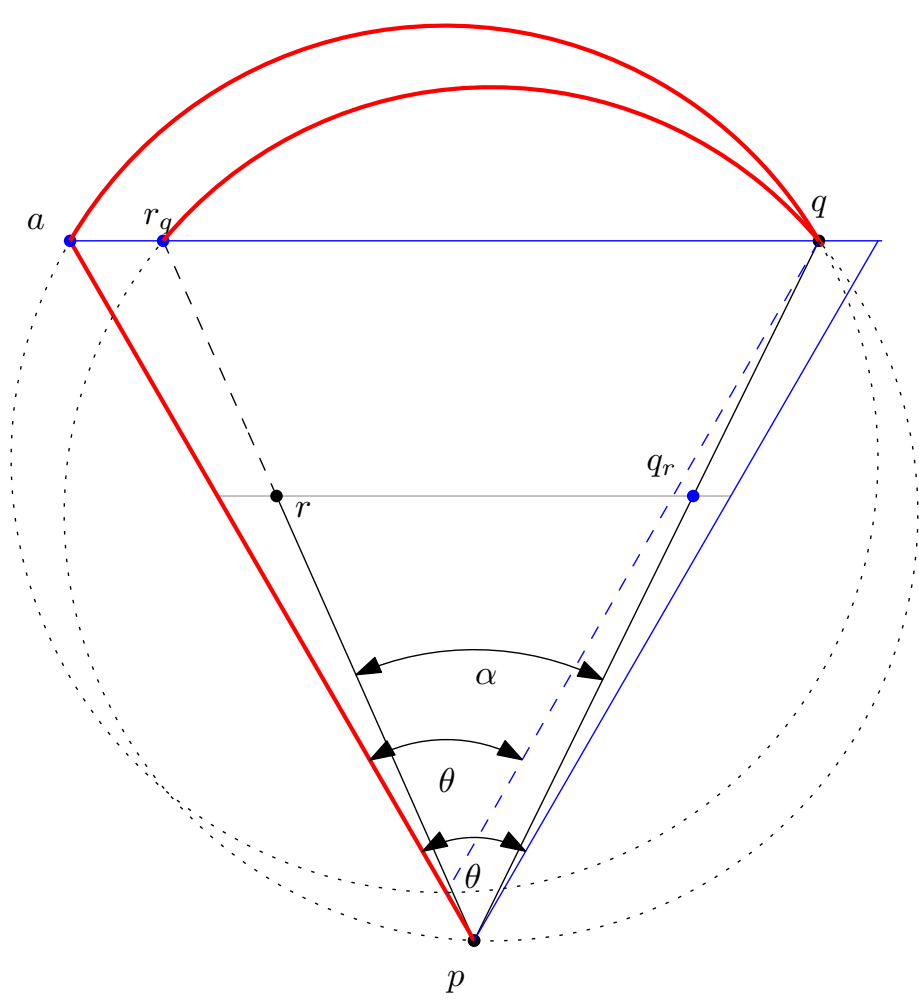

Figure 41: Lemma 5.2.8.

canonical subgraph $\mathrm{Can}_{i}^{(p, r)}$. Without loss of generality, assume that $i=0$. The length of the ideal path from $p$ to any vertex $q$ in $\operatorname{Can}_{0}^{(p, r)}$ is:

$$
\delta(r, q) \leq|p a|+\frac{\theta}{\sin \theta}|a q|
$$

where $a$ is the corner of $T_{p q}$ such that $r \in \triangle(p q a)$, and $\theta=\pi / 3$ is the angle of the cones.

Proof. By Lemma 5.2.7 the path from $r$ to $q$ is no greater than $\max \left\{\left|r r_{q}\right|,\left|q_{r} q\right|\right\}+\left|r_{q} q\right| \frac{\alpha}{\sin \alpha}$, where $\alpha=\angle(r p q)$. We have

$$
|p r|+\max \left\{\left|r r_{q}\right|,\left|q_{r} q\right|\right\} \leq|p a|
$$

Since $\frac{\alpha}{\sin \alpha}$ is an increasing function in the interval $0 \leq \alpha \leq \pi / 3, \theta>\alpha$ and $|a q|>\left|r_{q} q\right|$, 
we have

$$
\begin{aligned}
\delta(p, q) & \leq|p r|+\max \left\{\left|r r_{q}\right|,\left|q_{r} q\right|\right\}+\left|r_{q} q\right| \frac{\alpha}{\sin \alpha} \\
& \leq|p a|+|a q| \frac{\theta}{\sin \theta}
\end{aligned}
$$

as required.

See Figure 41 .

\subsection{Lengths of Paths in $D 8(P)$}

\subsubsection{Introduction}

In this section we prove the following theorem:

Theorem 5.4.2: For any edge $(p, q) \in D T(P)$, there is a path in $D 8(P)$ from $p$ to

$q$ with length at most $\left(1+\frac{\theta}{\sin \theta}\right)|p q|$, where $\theta=\pi / 3$ is the cone width. Thus $D 8(P)$ is a $\left(1+\frac{\theta}{\sin \theta}\right) D_{T}$-spanner of the complete graph, where $D_{T}$ is the spanning ratio of the Delaunay triangulation (currently 1.998 [7])

We first prove some supporting lemmas. Then we list the types of paths we can have between $p$ and $q$ in $D 8(P)$, where $(p, q)$ is an edge in $D T(P)$. Then we prove a bound of

$$
|p a|+\frac{\theta}{\sin \theta}|a q|
$$

on each type of path, where $(p, a)$ and $(a, q)$ are line segments on the border of the canonical triangle $T_{p q}$. The final type of path is the inductive path, and it encapsulates the other cases.

At this point our spanning ratio is with respect to $T_{p q}$. We then prove that $D 8(P)$ is a 


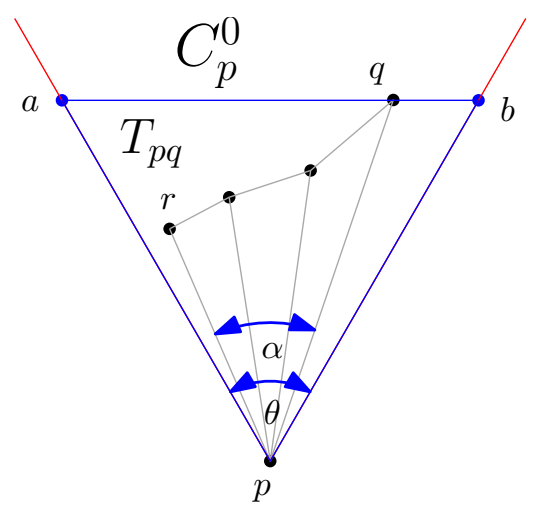

Figure 42: An example arrangement of vertices.

spanner with respect to the Euclidean distance $|p q|$.

\subsubsection{Preliminaries}

We consider an edge $(p, q) \in D T(P)$. If $(p, q) \in D 8(P)$ then the length of the path from $p$ to $q$ in $D 8(P)$ is $|p q| \leq\left(1+\frac{\theta}{\sin \theta}\right)|p q|$, as required.

Thus we assume $(p, q) \notin D 8(P)$. Without loss of generality we assume $q$ is in $C_{0}^{p}$. Since $(p, q) \notin D 8(P)$, there is an edge $(p, r)$ of $E_{A}$ in $\operatorname{Can}_{0}^{p}$ or $(q, u)$ in $\operatorname{Can}_{3}^{q}$ (or both), where $[p r] \leq[p q]$ and $[q u] \leq[p q]$. Otherwise $p$ and $q$ would have agreed to $(p, q)$, and it would have been added to $E_{A}$ in $\operatorname{AddIncident}(L)$. Without loss of generality we shall assume there is the edge $(p, r) \in E_{A},[p r] \leq[p q]$, and that $(p, q)$ is clockwise from $(p, r)$ around $p$.

Let $s$ be the vertex such that $s$ is a neighbour of $q$ in $N_{p}^{(p, r)}$ and $s \neq p$ (but possibly $s=r$ ). Let $a$ be the upper left corner of $T_{p q}$, and $b$ be the upper right corner. Let $\alpha=\angle(r p q)$ and $\theta=\pi / 3$ be the width of the cones. See Figure 42 for an example construction.

Lemma 5.3.1. Recall that $(p, r) \in E_{A}$, where $r \in C_{i}^{p}$. Then there is an ideal path from $p$ to any vertex $q$ in $\operatorname{Can}_{i}^{(p, r)}$, where $q$ is not an end vertex of $\operatorname{Can}_{i}^{p}$.

Proof. In the algorithm $A d d \operatorname{Canonical}(p, r)$, we add every canonical edge of $p$ in $\operatorname{Can}_{i}^{(p, r)}$ that is not the first or last edge. By Lemma 5.3.1. the edges of $\operatorname{Can}_{i}^{(p, r)}$ form a path. Thus 


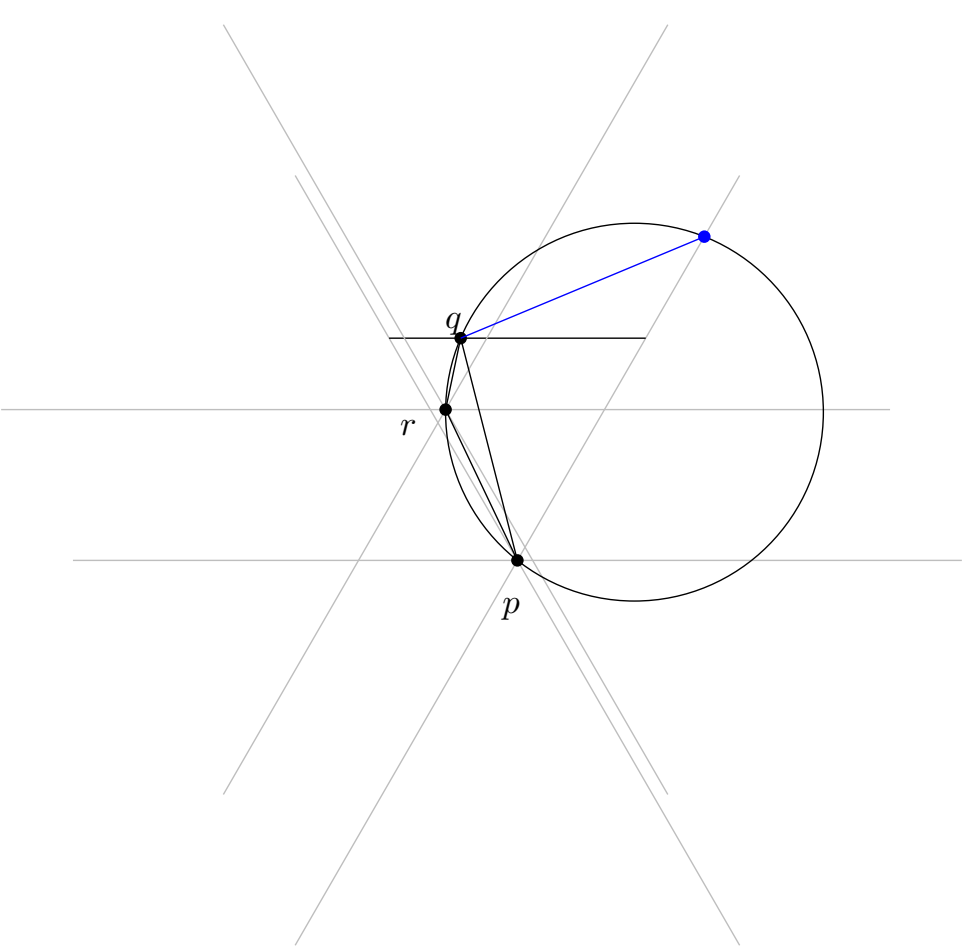

Figure 43: Any edge with endpoint $p$ in $N_{i}^{p}$ clockwise from $(p, q)$ must be longer than $[p q]$.

there is the ideal path from $p$ to any vertex $q$ in $\operatorname{Can}_{i}^{(p, r)}$ that is not the first or last vertex.

The next two lemmas prove that, for a vertex $z$ that is the first or the last vertex of $\operatorname{Can}_{i}^{p}$, that the edge in $\operatorname{Can}_{i}^{p}$ with endpoint $z$ cannot be in $C_{i}^{z}$.

Lemma 5.3.2. Let $r$ and $q$ be two consecutive neighbours of $p$, in an arbitrary cone $C_{i}^{p}$. Without loss of generality, let $(p, q)$ be clockwise from $(p, r)$ in the cone $C_{i}^{p}$. If $q$ is in $C_{i}^{r}$, then all edges with endpoint $p$ in $C_{i}^{p}$ that appear after $(p, q)$ in clockwise order are longer than $[p q]$.

Proof. By Lemma 4.3.8, any edge $(p, t)$ clockwise from $(p, q)$ in $C_{i}^{p}$ is such that the angle $\angle(r q t)>2 \pi / 3$. Since $(r, q)$ is in $C_{i}^{r}$, it is at an angle of at least $\pi / 3$ from the positive $x$-axis. Since $\angle(r q t)>2 \pi / 3$, the edge $(r, t)$ must be at an angle $>0$ with respect to the positive $x$-axis. Thus $[p t]>[p q]$, for all $(p, t)$ clockwise from $(p, q)$ in $C_{i}^{p}$. See Figure 43 


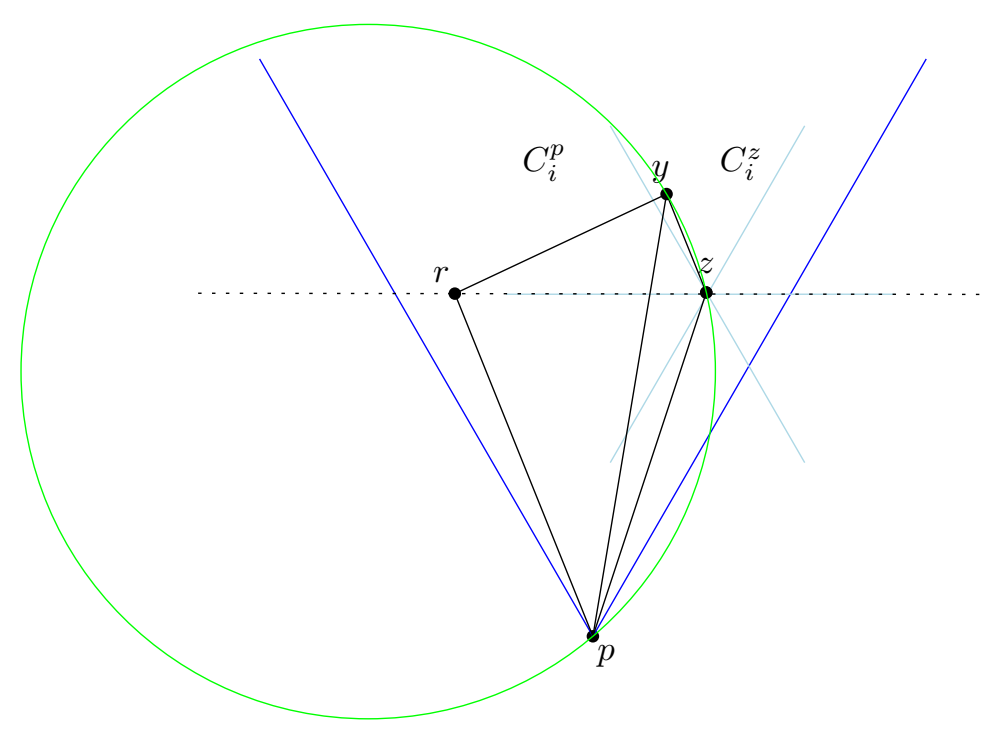

Figure 44: $y$ cannot be in $C_{i}^{z}$.

Lemma 5.3.3. Let $z$ be the first or last vertex of $\operatorname{Can}_{i}^{(p, r)}$, and assume that $(p, z)$ is not in $E_{A}$. Let $(y, z)$ be the last edge in $\operatorname{Can}_{i}^{(p, r)}$. Then $(y, z)$ is not in $C_{i}^{z}$.

Proof. We assume that $(y, z) \in C_{i}^{z}$, and prove by contradiction. By Lemma 5.3.2, if $(y, z)$ is in $C_{i}^{z}$, then $(p, y)$ is the shortest of all edges in $C_{i}^{p}$ with endpoint $p$ counter-clockwise from $(p, y)$.

Let $(p, r)$ be an edge in $E_{A}$, where $r \in \operatorname{Can}_{i}^{(p, r)}$. Then $(p, r)$ is at least as short as all edges in $D T(P)$ from $p$ to a vertex in $\operatorname{Can}_{i}^{(p, r)}$. But that is a contradiction to $(p, y)$ (and by extension $(p, z))$ being the shortest. See Figure 44

\subsubsection{Paths in D8(P)}

Let $(p, r)$ be an edge in $E_{A}$ is the graph $D 8(P)$. Without loss of generality, assume that $r$ is in $C_{0}^{p}$.

By Lemma 5.3.1, there is the ideal path from $p$ to any vertex in $\operatorname{Can}_{0}^{(p, r)}$ that is not the first or last vertex. We now turn our attention to the first or last vertex in $\operatorname{Can}_{0}^{(p, r)}$. Because the cases are symmetric, we focus on the last vertex, which we designate $z$. If $z=r$, the 


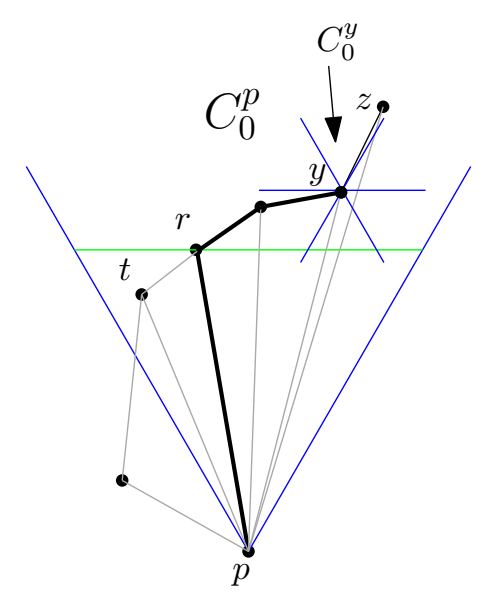

Figure 45: If the final edge of $\operatorname{Can}_{0}^{p}$ is in $C_{0}^{y}$, we do not add it to $E_{C A N}$.

path from $p$ to $z$ is trivial, thus we assume $z \neq r$. Let $y$ be the neighbour of $z$ in $\operatorname{Can}_{0}^{(p, r)}$. By Lemma 5.3.3. $(y, z)$ cannot be in $C_{0}^{z}$. Thus $(y, z)$ can be in $C_{5}^{z}, C_{4}^{z}$, or $C_{3}^{z}$.

Case 1: Edge $(y, z)$ is in $C_{5}^{z}$. Then $(y, z)$ was added to $E_{C A N}$ in $\operatorname{AddCanonical}(p, r), \operatorname{Step}$ 4 a, and there is an ideal path from $p$ to $z$.

Case 2: Edge $(y, z)$ is in $C_{4}^{z}$. There are three possibilities.

(a) If $(y, z)$ is an edge of $E_{A}$, then there is an ideal path from $p$ to $z$.

(b) If there is no edge in $E_{A}$ with endpoint $z$ in $C_{4}^{z}$, then $(y, z)$ was added to $E_{C A N}$ in $\operatorname{AddCanonical}(p, r)$, Step 4b, and there is an ideal path from $p$ to $z$.

(c) If there is an edge of $E_{A}$ in $C_{4}^{z}$ with endpoint $z$ that is not $(y, z)$, then we have added the canonical edge of $z$ in $C_{4}^{z}$ with endpoint $y$ to $E_{C A N}$ in AddCanonical $(p, r)$, Step 4c. Therefore by Lemma 5.3.1 there is an ideal path from $z$ to $y$, and also an ideal path from $p$ to $y$.

Case 3: Edge $(y, z)$ is in $C_{3}^{z}$. Then $(y, z)$ was not added to $E_{C A N}$. See Figure 45 .

In Case 1, Case 2a, and Case 2b there is an ideal path from $p$ to $q$. Thus Lemma 5.2.8 tells us there is a path from $p$ to $q$ not longer than $|p a|+\frac{\theta}{\sin \theta}|a q|$. 


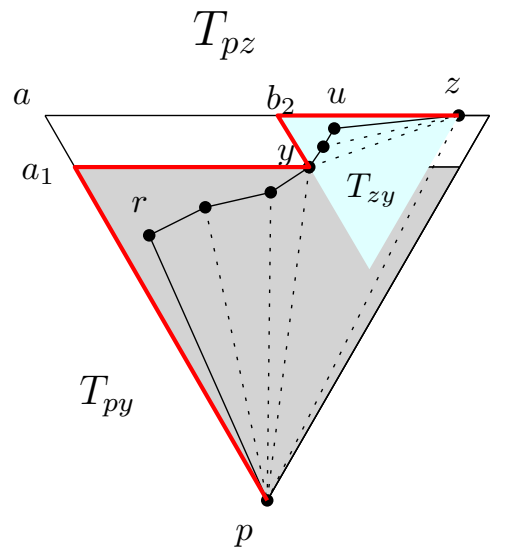

Figure 46: Ideal paths from $p$ to $y$ and $z$ to $y$.

In Case 2c, we have two ideal paths that meet at $y$. As in the case of a single ideal path, the sum of the lengths of these two paths is not more than $|p a|+\frac{\theta}{\sin \theta}|a q|$. The following lemma proves this claim:

Lemma 5.3.4. Consider the edge $(p, r)$ in $E_{A}$ in the graph $D 8(P), r$ in $C_{0}^{p}$. Let $(y, z)$ be the last edge in $\operatorname{Can}_{0}^{(p, r)}$, and let $(y, z)$ be in $C_{4}^{z}$. Let $(z, u)$ be an edge in $E_{A}$ in $C_{4}^{z}$. Assume there is an ideal path from $p$ to $y$ in $C_{0}^{p}$, and an ideal path from $z$ to $y$ in $C_{4}^{z}$. Let a be the top left corner of $T_{p z}$. We prove an upper bound on the length $\delta(p, z)$ :

$$
\delta(p, z) \leq|p a|+\frac{\theta}{\sin \theta}|a z|
$$

Proof. Let $a_{1}$ be the top left corner of $T_{p y}$, and let $b_{2}$ be the top right corner of $T_{z y}$ (as seen from apex $z$. Note that $T_{z y}$ lies in $\left.C_{z}^{4}\right)$. Since $(y, z)$ is the last edge in $\operatorname{Can}_{0}^{(p, r)}$, we note that the ideal path from $p$ to $y$ is to the side of $(p, y)$ that contains $r$ and does not contain $z$. Similarly, the ideal path from $z$ to $y$ is to the side of $(y, z)$ that contains $u$ and does not contain $p$. See Figure 46. By Lemma 5.3.1, the length of the path from $p$ to $z$ in $D 8(P)$ is: 


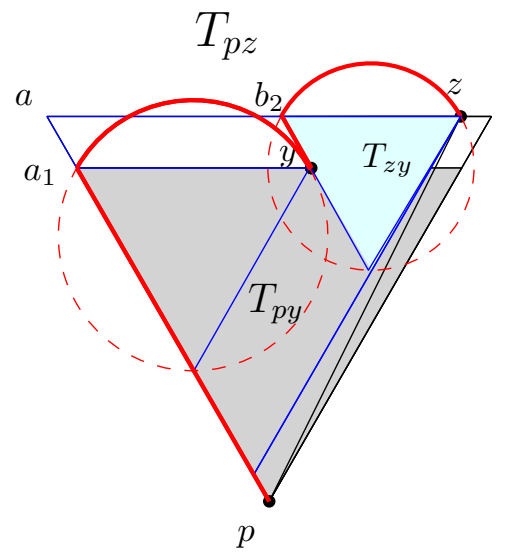

Figure 47: The path corresponding to Equation 5.3.1, Lemma 5.3.4.

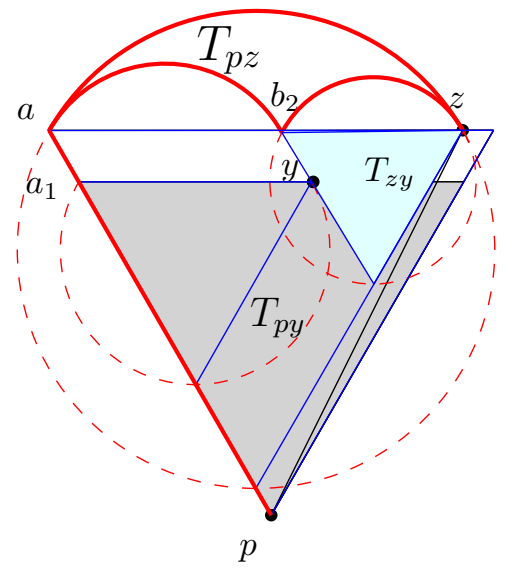

Figure 48: The path corresponding to Equations 5.3.2 and 5.3.3, in Lemma 5.3.4. 


$$
\begin{aligned}
\delta_{D 8(P)}(p, z) & \leq \delta_{D 8(P)}(p, y)+\delta_{D 8(P)}(z, y) \\
& \leq\left|p a_{1}\right|+\frac{\theta}{\sin \theta}\left|a_{1} s\right|+\left|z b_{2}\right|+\frac{\theta}{\sin \theta}\left|b_{2} y\right| \\
& \leq\left|p a_{1}\right|+\frac{\theta}{\sin \theta}\left|a_{1} s\right|+\left|b_{2} y\right|+\frac{\theta}{\sin \theta}\left|z b_{2}\right| \\
& \leq\left(\left|p a_{1}\right|+\left|b_{2} y\right|\right)+\frac{\theta}{\sin \theta}\left(\left|a_{1} y\right|+\left|z b_{2}\right|\right) \\
& =|p a|+\frac{\theta}{\sin \theta}|a z|
\end{aligned}
$$

Inequality 5.3.1 holds because $\frac{\theta}{\sin \theta}>1$, and $\left|b_{2} y\right| \leq\left|z b_{2}\right|$, since $\left|z b_{2}\right|$ is the longest possible line segment in $T_{z y}$. See Figures 46, 47, and 48 .

In Case 3 there is no edge from $y$ to $z$. We prove the length of the path from $p$ to $z$ in Case 3 by induction, as part of the main lemma of this section:

Lemma 5.3.5. Consider the edge $(p, r)$ in $E_{A}$ in the graph $D 8(P)$. Without loss of generality, let $r$ be in $C_{0}^{p}$. Let $a$ and $b$ be the top left corner and top right corner respectively of $T_{p q}$. For any edge $(p, q) \in D T(P)$, there exists a path from $p$ to $q$ in $D 8(P)$ that is not longer than:

$$
\max \left\{|p a|+\frac{\theta}{\sin \theta}|a q|,|p b|+\frac{\theta}{\sin \theta}|b q|\right\}
$$

Proof. Let $\delta(p, q)$ be the shortest path from $p$ to $q$ in $D 8(P)$. We do a proof by induction on the size of the canonical triangle $T_{p q}$.

The base case is when $T_{p q}$ is the smallest canonical triangle. One instance of this occurs when there is an ideal path from $p$ to $q$, as in Case 1, Case 2a, and Case 2b. Thus by Lemma 5.2 .8

$$
\delta(p, q) \leq|p a|+\frac{\theta}{\sin \theta}|a q|
$$




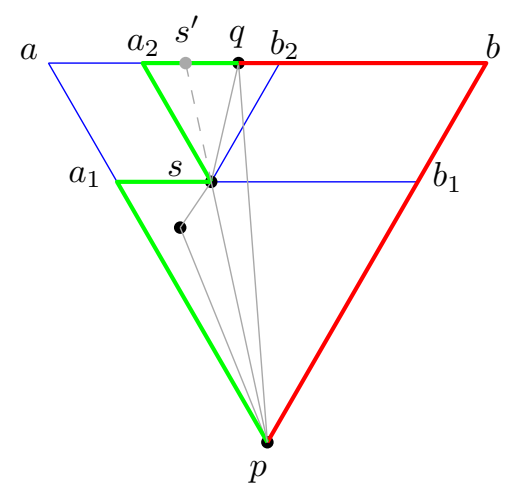

Figure 49: The green path is the length of the path we can prove, the red path is how much path we have to pay for it.

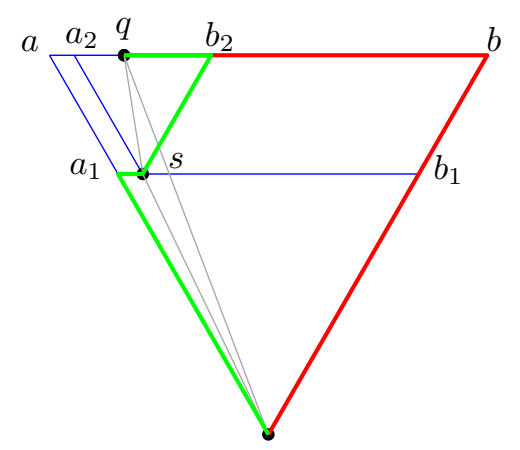

Figure 50: Notice that the inductive path takes the longer path between $a_{2}$ and $b_{2}$.

The other instance is Case 2c, where two ideal paths meet at a vertex. By Lemma 5.3.4 we have:

$$
\delta(p, q) \leq|p a|+\frac{\theta}{\sin \theta}|a q|
$$

Since $|a q| \leq \max \{|a q|,|b q|\}$, the proof holds in all base cases.

In Case 3, $q$ is the first or last vertex in $\operatorname{Can}_{0}^{(p, r)}$. Since the cases are symmetric, consider when $q$ is the last vertex, and assume it has a neighbour $s$ in $\operatorname{Can}_{0}^{(p, r)}$, such that the canonical edge $(s, q)$ in $N_{0}^{p}$ is in $C_{0}^{s}$. Thus $(s, q)$ was not added to $E_{C A N}$ on a call to $\operatorname{AddCanonical}(p, r)$.

We break down $T_{p q}$ into canonical triangles $T_{p s}$ and $T_{s q}$. Call the upper left corner of $T_{p q} a$, and the upper right corner $b$. Also the upper left corner of $T_{p s}$ is $a_{1}$, the upper right corner of $T_{s q}$ is $a_{2}$, the upper right corner of $T_{p s}$ is $b_{1}$, and the upper right corner of $T_{s q}$ is $b_{2}$. Since $(s, q)$ is in $C_{0}^{s}$, both $T_{p s}$ and $T_{s q}$ must be smaller than $T_{p q}$. 


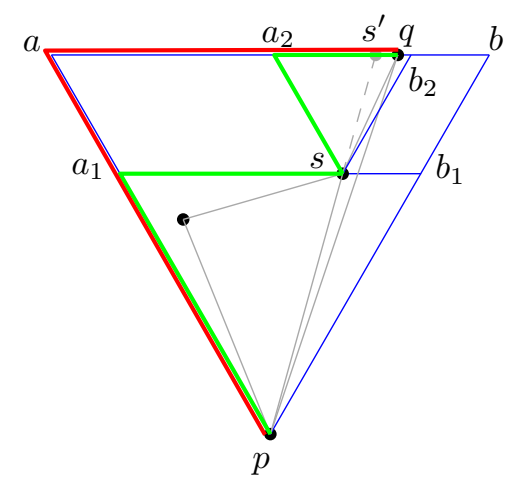

Figure 51: $\left|a_{1} s\right| \geq\left|s b_{1}\right|$.

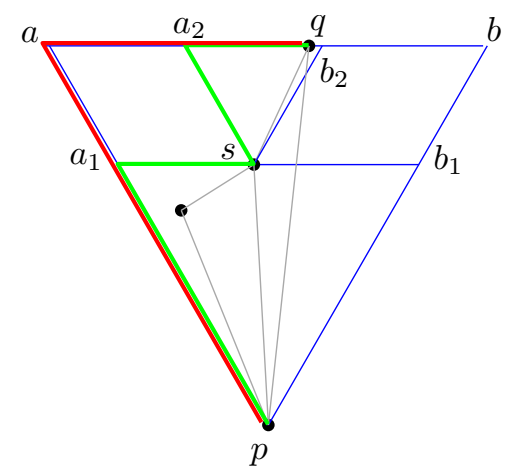

Figure 52: $\left|a_{1} s\right| \geq\left|s b_{1}\right|$. 
We note the following facts:

Fact 1: $|p a|=\left|p a_{1}\right|+\left|s a_{2}\right|$ and likewise $|p b|=\left|p b_{1}\right|+\left|s b_{2}\right|$

Fact 2: $|a b|=\left|a_{1} b_{1}\right|+\left|a_{2} b_{2}\right|$

Fact 3: $\left|a a_{2}\right|=\left|a_{1} s\right|$ and $\left|b_{2} b\right|=\left|s b_{1}\right|$

Fact 4: $q$ is on the line $\left(a_{2}, b_{2}\right)$

Without loss of generality, assume the path from $p$ to $s$ is to the side of the line through $p$ and $s$ with $a_{1}$ (note that we are not assuming that $\left.\left|a_{1} s\right|>\left|b_{1} s\right|\right)$.

We extend the line $(p, s)$ until it intersects $\left(a_{2}, b_{2}\right)$ at a point we label $s^{\prime}$. Since $q$ is the last vertex in $\operatorname{Can}_{0}^{(p, r)}, q$ must be to the side of $s^{\prime}$ closer to $b_{2}$.

Since $|p a|=|p b|$ and $\left|s a_{2}\right|=\left|s b_{2}\right|$, it is sufficient to prove:

$$
\left|p a_{1}\right|+\frac{\theta}{\sin \theta}\left|a_{1} s\right|+\left|s a_{2}\right|+\frac{\theta}{\sin \theta} \max \left\{\left|a_{2} q\right|,\left|q b_{2}\right|\right\} \leq|p a|+\frac{\theta}{\sin \theta} \max \{|a q|,|b q|\}
$$

By Fact 1 this is equivalent to:

$$
\begin{aligned}
\frac{\theta}{\sin \theta}\left|a_{1} s\right|+\frac{\theta}{\sin \theta} \max \left\{\left|a_{2} q\right|,\left|q b_{2}\right|\right\} & \leq \frac{\theta}{\sin \theta} \max \{|a q|,|b q|\} \\
\left|a_{1} s\right|+\max \left\{\left|a_{2} q\right|,\left|q b_{2}\right|\right\} & \leq \max \{|a q|,|q b|\}
\end{aligned}
$$

We consider two scenarios:

1. $\left|a_{1} s\right| \leq\left|s b_{1}\right|:$ There are two sub-cases:

(a) $|q b| \geq|a q|$ : If $\left|a_{2} q\right| \geq\left|q b_{2}\right|$, then:

$$
\begin{aligned}
\left|a_{1} s\right|+\left|a_{2} q\right| & \leq|a q| \\
& \leq|q b|
\end{aligned}
$$


as required. Otherwise, $\left|q b_{2}\right|>\left|a_{2} q\right|$, thus:

$$
\begin{aligned}
\left|a_{1} s\right|+\left|q b_{2}\right| & \leq\left|s b_{1}\right|+\left|q b_{2}\right| \\
& =|q b|
\end{aligned}
$$

as required.

(b) $|q b|<|a q|$ : Together with $\left|a_{1} s\right| \leq\left|s b_{1}\right|$ implies that $\left|a_{2} q\right|>\left|q b_{2}\right|$. See Figure 52 . Then $\left|a_{1} s\right|+\left|a_{2} q\right|=|a q|$, as required.

2. $\left|a_{1} s\right|>\left|s b_{1}\right|$ : Since $q$ is radially to the right of $(p, s),|a q|>|q b|$. It is also true that $\left|a_{2} q\right|>\left|q b_{2}\right|$. Thus, using Fact 3.

$$
\begin{aligned}
\left|a_{1} s\right|+\left|a_{2} q\right| & =\left|a a_{2}\right|+\left|a_{2} q\right| \\
& =|a q|
\end{aligned}
$$

as required. See Figure 51.

\subsection{The Spanning Ratio of D8(P)}

For an edge $(p, q)$ in $D T(P)$, we have a bound on the length of the path in $D 8(P)$. However, this bound is terms of the size of the canonical triangle $T_{p q}$, which is not the same as the Euclidean distance $|p q|$.

We prove that $\left(1+\frac{\theta}{\sin \theta}\right)|p q|$ is actually an upper bound on $|p a|+\frac{\theta}{\sin \theta}|a q|$ here:

\section{Claim 5.4.1.}

$$
\max \left\{|p a|+\frac{\theta}{\sin \theta}|a q|,|p b|+\frac{\theta}{\sin \theta}|b q|\right\} \leq\left(1+\frac{\theta}{\sin \theta}\right)|p q|
$$


Proof. Without loss of generality, we will assume that

$$
\max \left\{|p a|+\frac{\theta}{\sin \theta}|a q|,|p b|+\frac{\theta}{\sin \theta}|b q|\right\}=|p a|+\frac{\theta}{\sin \theta}|a q|
$$

Let

$$
\lambda=\left(\frac{\theta}{\sin \theta}-1\right)(|p q|-|a q|)
$$

We will show that:

$$
|p a|+\frac{\theta}{\sin \theta}|a q| \leq|p a|+\frac{\theta}{\sin \theta}|a q|+\lambda \leq\left(1+\frac{\theta}{\sin \theta}\right)|p q|
$$

Since $|p q| \geq|p a|$ (by the sine law), and $\frac{\theta}{\sin \theta}>1$, we get $\lambda \geq 0$. Thus

$$
\begin{gathered}
|p a|+\frac{\theta}{\sin \theta}|a q| \\
\leq|p a|+\frac{\theta}{\sin \theta}|a q|+\lambda
\end{gathered}
$$

It remains to be shown that: 


$$
\begin{aligned}
|p a|+\frac{\theta}{\sin \theta}|a q|+\lambda & \leq\left(1+\frac{\theta}{\sin \theta}\right)|p q| \\
|p a|+\frac{\theta}{\sin \theta}|a q|+\left(\frac{\theta}{\sin \theta}-1\right)(|p q|-|a q|) & \leq\left(1+\frac{\theta}{\sin \theta}\right)|p q| \\
|p a|-|p q|+|a q|+\frac{\theta}{\sin \theta}(|a q|+|p q|-|a q|) & \leq\left(1+\frac{\theta}{\sin \theta}\right)|p q| \\
|p a|-|p q|+|a q|+\frac{\theta}{\sin \theta}|p q| & \leq|p q|+\frac{\theta}{\sin \theta}|p q| \\
|p a|-|p q|+|a q| & \leq|p q| \\
|p a|+|a q| & \leq 2|p q|
\end{aligned}
$$

Thus we must show that $|p a|+|a q| \leq 2|p q|$ holds true for all values of $\alpha=\angle(a p q)$.

Let $a^{\prime}$ be the point to the side of $(p, q)$ that contains $a$, such that $\triangle\left(a^{\prime} p q\right)$ is an equilateral triangle. Thus

$$
\left|p a^{\prime}\right|+\left|a^{\prime} q\right|=2|p q|
$$

See Figure 53b. We will prove that

$$
|p a|+|a q| \leq\left|p a^{\prime}\right|+\left|a^{\prime} q\right|=2|p q| .
$$

Note that $\angle(p a q)=\angle\left(p a^{\prime} q\right)=\theta$. That implies that the circle $O_{p a^{\prime} q}$ through $p, a^{\prime}$ and $q$ also goes through $a$. See Figure $53 \mathrm{c}$.

To better analyze the problem, we rotate, translate, and scale $p, q, a$ and $a^{\prime}$ such that $q=(-\sin \theta, 0), p=(\sin \theta, 0)$, and $a^{\prime}=(0,-1.5)$. Let $a$ be any point on $O_{p q a^{\prime}}$ below the line through $p$ and $q$. Let $E(p, q, d)$, where $d=2|p q|$, represent an ellipse with focal points $p$ and $q$ such that for each point $b$ on the boundary of $E(p, q, d),|p b|+|b q|=d=2|p q|$. Note the center of $O_{p q a^{\prime}}$ is $(0,-0.5)$, and $O_{p q a^{\prime}}$ has a radius of 1 . See Figure $53 \mathrm{~d}$. The equation for $O_{p q a^{\prime}}$ is: 


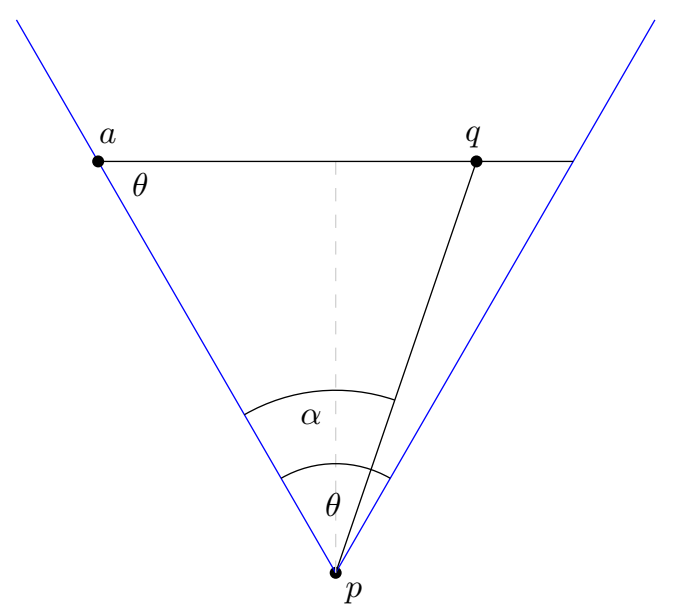

(a)

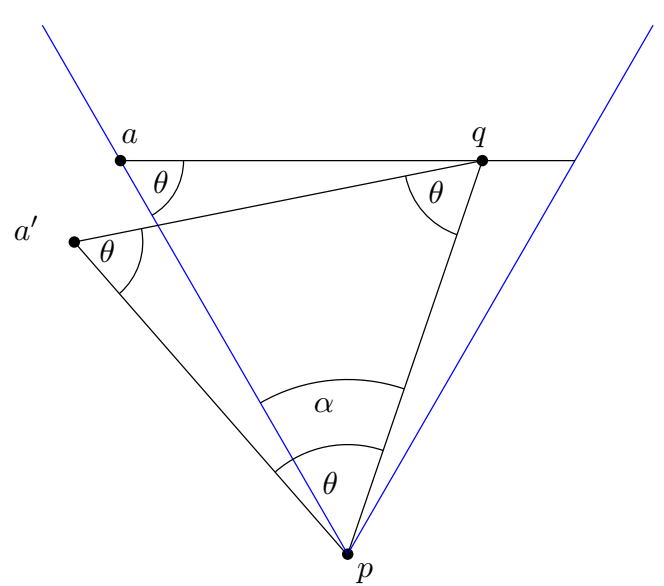

(b)

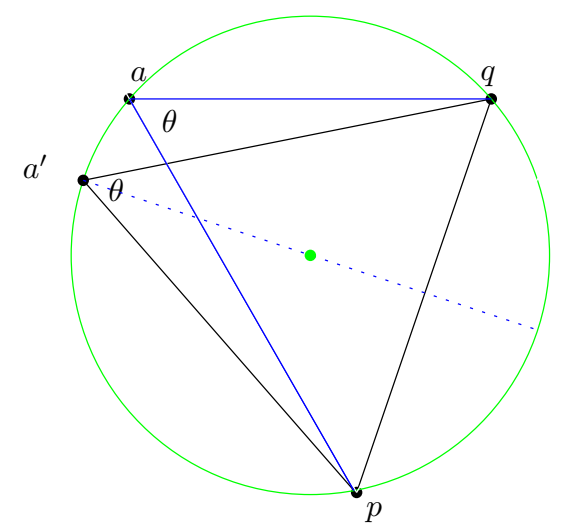

(c) Circle $O_{p q a^{\prime}}$ also goes through $a$.

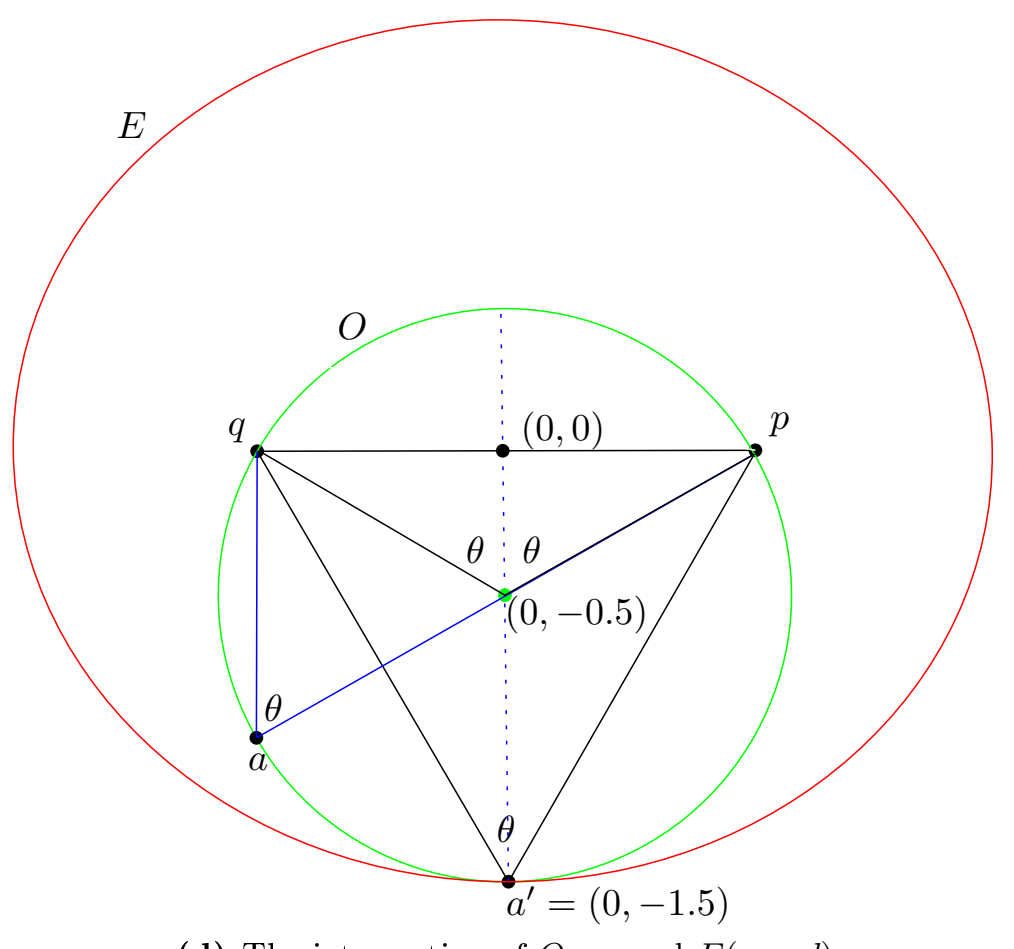

(d) The intersection of $O_{p q a^{\prime}}$ and $E(p, q, d)$.

Figure 53: AddCanonical $(p, r)$ 


$$
x^{2}+\left(y+\frac{1}{2}\right)^{2}=1
$$

The equation for $E(p, q, d)$ is:

$$
\begin{aligned}
\frac{x^{2}}{a^{2}}+\frac{y^{2}}{b^{2}} & =1 \\
\frac{x^{2}}{(2 \sin \theta)^{2}}+\frac{y^{2}}{\frac{3}{2}^{2}} & =1 \\
\frac{x^{2}}{3}+\frac{4 y^{2}}{9} & =1
\end{aligned}
$$

Thus we find the intersection of $O_{p q a^{\prime}}$ and $E(p, q, d)$ by solving the following system of equations:

$$
\begin{aligned}
x^{2}+\left(y+\frac{1}{2}\right)^{2} & =1 \\
\frac{x^{2}}{3}+\frac{4 y^{2}}{9} & =1
\end{aligned}
$$

This gives us a single solution at $(0,-1.5)$.

Note that, when $\angle(a q p)=\pi / 2,|p a|=2$ and $|a q|=2 \cos \theta=1$. Thus $|p a|+|a q|=3$.

We have $2|p q|=2 *(2 \sin \theta) \approx 3.46$. Thus when $\angle(a q p)=\pi / 2,|p a|+|a q|<2|p q|=$ $\left|p a^{\prime}\right|+\left|a^{\prime} q\right|$, which means that $a$ is inside $E(p, q, d)$, which means all of $O_{p q a^{\prime}}$ is inside $E(p, q, d)$, with the exception of $(0,-1.5)$. Thus for all points $a$ on $O_{p q a^{\prime}}$,

$$
|p a|+|a q| \leq\left|p a^{\prime}\right|+\left|a^{\prime} q\right|=2|p q|
$$

Which implies that: 


$$
\delta(p, q) \leq|p a|+\frac{\theta}{\sin \theta}|a q| \leq\left(1+\frac{\theta}{\sin \theta}\right)|p q|
$$

as required.

We can now prove the main theorem:

Theorem 5.4.2. For any edge $(p, q) \in D T(P)$, there is a path in $D 8(P)$ from $p$ to $q$ with length at most $\left(1+\frac{\theta}{\sin \theta}\right)|p q|$, where $\theta=\pi / 3$ is the cone width. Thus $D 8(P)$ is a $\left(1+\frac{\theta}{\sin \theta}\right) D_{T^{-}}$spanner of the complete graph, where $D_{T}$ is the spanning ratio of the Delaunay triangulation (currently 1.998 [7]).

Proof. Follows from Lemma 5.3.5 and Claim 5.4.1. 


\section{Chapter 6}

\section{Conclusion and Future Work}

We have given a construction for a graph $D 8(P)$ that has a degree of 8 , which is low enough to be practical in many applications, while still maintaining a competitive spanning ratio of $\approx 4.41$, which is the best known spanning ratio of a planar graph with a degree bound less than 14 .

To build and analyze this graph, we borrow heavily from the techniques used by Kanj et. al [3] in their analysis of a bounded degree spanning subgraph of the Delaunay triangulation. Additionally we reuse techniques and analysis used by Bonichon et. al [1] in their degree 6 spanning subgraph of half- $\theta-6$ graphs. The combination of these techniques gives a spanning ratio that, when adjusted for cone angle, matches the best known. We get an improvement

on the spanning ratio of the graphs of Bonichon et. al [1], which shows the difference a strong underlying graph can make.

Although our construction and analysis involves a few more edge cases than either Bonichon et. al [1] or Kanj et. al [3], it does demonstrate the flexibility of the techniques involved. Perhaps they could be applied to other variations of graphs, or variations of the Delaunay triangulation or the half- $\theta-6$ graph.

While incredibly useful, the analysis of Kanj et. al [3], and by extension our analysis, is quite technical. The paper by Bose et. al [2] provides the model for the algorithm that constructs $D 8(P)$, and they use an algorithm with a subroutine similar to our AddIncident(). However, rather than sorting the edges by bisector distance, they use Euclidean distance. 
This allows them a huge simplification in the analysis of the subsequent graph over what we achieve. Perhaps some similar simplification of the analysis of our graph is possible, either by modifying the construction, or by providing a more clever analysis technique.

In the paper by Bonichon et al, [1], they first develop a degree 9 graph, and then use that graph to transition into a degree 6 graph. Originally we had meant to follow in their footsteps and try something similar. That is, we wished to use $D 8(P)$ as a transition into a degree 6 graph with a similar, if not identical, spanning ratio. As such a degree 6 graph is a possible next step.

Always of interest in the graph setting, a generalized or constrained version of this graph could prove interesting. Bose et al. 14 have applied constraints to the graph developed by Bonichon et al, [1]. Having borrowed many of their techniques and applied them successfully on a Delaunay triangulation, it seems apropos to attempt to apply their constrained techniques to our setting as well. 


\section{List of References}

[1] N. Bonichon, C. Gavoille, N. Hanusse, and L. Perković. "Plane spanners of maximum degree six." In S. Abramsky, C. Gavoille, C. Kirchner, F. Meyer auf der Heide, and P. Spirakis, editors, "Automata, Languages and Programming," volume 6198 of Lecture Notes in Computer Science, pages 19-30. Springer Berlin Heidelberg. ISBN 978-3-64214164-5 (2010).

[2] P. Bose, P. Carmi, and L. Chaitman-Yerushalmi. "On bounded degree plane strong geometric spanners." Journal of Discrete Algorithms 15(0), 16 - 31. ISSN 1570-8667 (2012).

[3] I. A. Kanj, L. Perković, and G. Xia. "On spanners and lightweight spanners of geometric graphs." SIAM Journal on Computing 39(6), 2132-2161 (2010).

[4] P. Chew. "There is a planar graph almost as good as the complete graph." In "Proceedings of the Second Annual Symposium on Computational Geometry," SCG '86, pages 169-177. ACM, New York, NY, USA. ISBN 0-89791-194-6 (1986).

[5] D. Dobkin, S. Friedman, and K. Supowit. "Delaunay graphs are almost as good as complete graphs." Discrete \& Computational Geometry 5(1), 399-407. ISSN 0179-5376 (1990).

[6] J. Keil and C. Gutwin. "Classes of graphs which approximate the complete euclidean graph." Discrete $\&$ Computational Geometry 7(1), 13-28. ISSN 0179-5376 (1992).

[7] G. Xia. "Improved upper bound on the stretch factor of Delaunay triangulations." In "Proceedings of the Twenty-seventh Annual Symposium on Computational Geometry," SoCG '11, pages 264-273. ACM, New York, NY, USA. ISBN 978-1-4503-0682-9 (2011).

[8] P. Bose, L. Devroye, M. Löffler, J. Snoeyink, and V. Verma. "Almost all Delaunay triangulations have stretch factor greater than $\pi / 2 . "$ Comput. Geom. Theory Appl. 44(2), 121-127. ISSN 0925-7721 (2011). 
[9] G. Xia and L. Zhang. "Toward the tight bound of the stretch factor of Delaunay triangulations." In "Proceedings of the Canadian Conference on Computational Geometry," CCCG'11 (2011).

[10] P. Bose, J. Gudmundsson, and M. Smid. "Constructing plane spanners of bounded degree and low weight." In R. MÂúhring and R. Raman, editors, "Algorithms - ESA 2002," volume 2461 of Lecture Notes in Computer Science, pages 234-246. Springer Berlin Heidelberg. ISBN 978-3-540-44180-9 (2002).

[11] X.-Y. Li and Y. Wang. "Efficient construction of low weight bounded degree planar spanner." In T. Warnow and B. Zhu, editors, "Computing and Combinatorics," volume 2697 of Lecture Notes in Computer Science, pages 374-384. Springer Berlin Heidelberg. ISBN 978-3-540-40534-4 (2003).

[12] P. Bose, M. H. M. Smid, and D. Xu. "Delaunay and diamond triangulations contain spanners of bounded degree." Int. J. Comput. Geometry Appl. pages 119-140 (2009).

[13] N. Bonichon, I. Kanj, L. Perković, and G. Xia. "There are plane spanners of degree 4 and moderate stretch factor." Discrete \& Computational Geometry 53(3), 514-546. ISSN 0179-5376 (2015).

[14] P. Bose, R. Fagerberg, A. van Renssen, and S. Verdonschot. "On plane constrained bounded-degree spanners." In "Proceedings of the 10th Latin American International Conference on Theoretical Informatics," LATIN'12, pages 85-96. Springer-Verlag, Berlin, Heidelberg. ISBN 978-3-642-29343-6 (2012).

[15] R. Benson. Euclidean geometry and convexity. McGraw-Hill (1966).

[16] P. Bose and J. M. Keil. "On the stretch factor of the constrained Delaunay triangulation." In "3rd International Symposium on Voronoi Diagrams in Science and Engineering, ISVD 2006, Banff, Alberta, Canada, July 2-5, 2006," pages 25-31. IEEE Computer Society (2006). 